\title{
THE RATIONALITY PROBLEM FOR CONIC BUNDLES
}

\author{
YURI PROKHOROV
}

\begin{abstract}
This expository paper is concerned with the rationality problems for three-dimensional algebraic varieties with a conic bundle structure. We discuss the main methods of this theory. We sketch the proofs of certain principal results, and present some recent achievements. Many open problems are also stated.
\end{abstract}

\section{Contents}

1. Introduction

2. Preliminaries

3. Conic bundles

4. Sarkisov category 15

5. Surfaces over non-closed fields 18

6. The Artin-Mumford invariant 23

7. Intermediate Jacobians and Prym varieties 26

8. Birational transformations 30

9. Conic bundles over minimal surfaces 41

10. $\mathbb{Q}$-conic bundles 44

11. Examples of Sarkisov links on $\mathbb{Q}$-conic bundles 51

12. Birational transformations of $\mathbb{Q}$-conic bundles, I 56

13. Birational transformations of $\mathbb{Q}$-conic bundles, II 5

14. Some related results and open problems 68

References

\section{INTRODUCTION}

We basically work over the field $\mathbb{C}$ of complex numbers. In this paper we deal with algebraic varieties having a structure of conic bundle over surfaces. A motivation for the study above is that conic bundles

This work was partially supported by the Russian Academic Excellence Project "5-100". The paper was written while the author was visiting the Max Planck Institute for Mathematics (Bonn). He would like to thank the institute for the invitation and excellent working condition.

Submitted to Uspeshi Mat. Nauk = Russian Math. Surveys. 
occur in the birational classification of threefolds of negative Kodaira dimension.

According to the minimal model program [Mor88, [BCHM10] every uniruled algebraic projective variety $Y$ is birationally equivalent to a projective variety $X$ with at most $\mathbb{Q}$-factorial terminal singularities that admits a contraction $\pi: X \rightarrow S$ to a lower-dimensional normal projective variety $S$ such that the anticanonical divisor $-K_{X}$ is $\pi$ ample and

$$
\operatorname{Pic}(X)=\pi^{*} \operatorname{Pic}(S) \oplus \mathbb{Z}
$$

1.1. In dimension 3 there are the following three possibilities:

- $S$ is a point and then $X$ is called a $\mathbb{Q}$-Fano threefold;

- $S$ is a smooth projective curve and then $\pi: X \rightarrow S$ is called a $\mathbb{Q}$-del Pezzo fibration;

- $S$ is a normal surface and then $\pi: X \rightarrow S$ is called a $\mathbb{Q}$-conic bundle.

$\mathbb{Q}$-Fano threefolds are bounded [Kaw92] (this is true even in arbitrary dimension [Bir16a]). However, an explicit classification is known only in three-dimensional case for smooth ones [IP99]. There are a lot of partial results related to singular $\mathbb{Q}$-Fano threefolds, see e.g. Ale94b], [B ${ }^{+}$, [PR16], Pro15a, [Pro16], [Pro17], and references therein.

For $\mathbb{Q}$-del Pezzo fibrations there are partial results on construction of standard models Cor96, [Kol97 and birational rigidity, see e.g. [Puk98, Puk13, Ch. 4-5], Che05, Sob02, Gri00, SC11.

In this paper we concentrate on the last case of 1.1. We are mainly interested in rationality questions. It is known that any $\mathbb{Q}$-conic bundle has a standard model, that is, a $\mathbb{Q}$-conic bundle $\pi^{\bullet}: X^{\bullet} \rightarrow S^{\bullet}$ such that the total space $X^{\bullet}$ and the surface $S^{\bullet}$ are smooth and there are birational maps $X^{\bullet} \rightarrow X$ and $S^{\bullet} \rightarrow S$ making the corresponding diagram commutative (see Theorem 3.12). The following conjecture is motivated by rationality criterion for two-dimensional conic bundles (see Sect. 51).

1.2. Conjecture ([Sho84]). Let $\pi: X \rightarrow S$ be a standard conic bundle over a rational surface $S$ with discriminant curve $\Delta \subset S$. In this case, $X$ is rational if and only if the following holds:

$(\star)\left|2 K_{S}+\Delta\right|=\varnothing$ and, in the case $\mathrm{p}_{\mathrm{a}}(\Delta)=6$, the Griffiths component $\mathrm{J}_{\mathrm{G}}(X)$ of the intermediate Jacobian $\mathrm{J}(X)$ is trivial.

Note that in the case $\left|2 K_{S}+\Delta\right|=\varnothing$ and $\mathrm{p}_{\mathrm{a}}(\Delta)=6$ the variety $X$ can be non-rational (i.e. the condition $\mathrm{J}_{\mathrm{G}}(X)=0$ here cannot be removed). In fact, in this case $\pi: X \rightarrow S$ is fiberwise birational to a standard conic bundle $\pi^{\sharp}: X^{\sharp} \rightarrow \mathbb{P}^{2}$ with discriminant curve $\Delta^{\sharp}$ of 
degree 5 so that the corresponding double cover $\tilde{\Delta}^{\sharp} \rightarrow \Delta^{\sharp}$ is defined by an odd theta characteristic (see Corollary 8.7.1). Moreover, $X$ is birational to a three-dimensional cubic hypersurface (see Proposition 8.9).

1.3. Conjecture ([Isk87]). Let $\pi: X \rightarrow S$ be a standard conic bundle over a rational surface $S$ with discriminant curve $\Delta \subset S$. In this case, $X$ is rational if and only if one of the following conditions is satisfied.

(i) There exists a commutative diagram

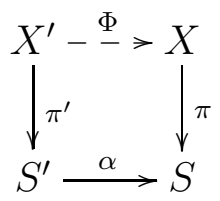

where $\pi^{\prime}: X^{\prime} \rightarrow S^{\prime}$ is a standard conic bundle with a discriminant curve $\Delta^{\prime} \subset S^{\prime}, \alpha$ is a birational morphism, and $\Phi$ is a birational map, and a base point free pencil of rational curves $\mathscr{L}^{\prime}$ on $S^{\prime}$ such that $\mathscr{L}^{\prime} \cdot \Delta^{\prime} \leq 3$.

(ii) There exists a commutative diagram

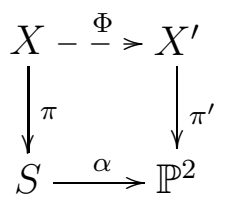

where $\pi^{\prime}: X^{\prime} \rightarrow S^{\prime}=\mathbb{P}^{2}$ is a standard conic bundle with discriminant curve $\Delta^{\prime} \subset \mathbb{P}^{2}$ of degree $5, \alpha$ is a birational morphism, $\Phi$ is a birational map, and $X^{\prime}$ is a blow-up of $\mathbb{P}^{3}$ along a non-singular curve $\Gamma \subset \mathbb{P}^{3}$ of genus 5 and degree 7 (see Example 3.4.3).

It is not so difficult to show that Conjectures 1.3 and 1.2 are equivalent, see Corollary 8.7.2. The sufficiency of (i) and (ii) in Conjecture 1.3 and $(\star)$ in Conjecture 1.2 is also known (see Propositions 8.1 and 5.6 and Corollary 8.7.1). The hardest part of these conjectures is the necessity. The necessity of 1.3](i) (ii) and 1.2](*) was proved by Shokurov in the case of conic bundles over minimal rational surfaces (see Sect. 9).

1.4. Remark. Note that the condition (i) of Conjecture 1.3 can be replaced with the following (see Proposition 8.6):

$\left(\mathrm{i}^{\prime}\right) \pi$ is fiberwise birationally equivalent to a standard conic bundle $\pi^{\sharp}: X^{\sharp} \rightarrow S^{\sharp}$, where $S^{\sharp}=\mathbb{F}_{n}$ is a rational geometrically ruled surface and the discriminant curve $\Delta^{\sharp}$ meets a general fiber in at most three points. 
The paper is organized as follows. Sections 2 and 3 are preliminary. There we fix the notation, give basic definitions, and collect general facts about varieties with conic bundle structures. In Section 4 we introduce the Sarkisov program of decomposition of birational maps between Mori fiber spaces. The proof of the main theorem in the threedimensional case is outlined. Section 5 is about surface conic bundles over algebraically non-closed fields. There we recall the classification of two-dimensional Sarkisov links and formulate rationality criterion. This theory is a motivation for the main conjectures 1.2 and 1.3 , as well as, one of the main tools in the proofs.

There are two important birational invariants of algebraic varieties that use transcendental and topological methods: torsions in the middle cohomology groups and the intermediate Jacobian. These notions are discussed in Sections 6 and 7, respectively. In Section 8 we collect examples of some special Sarkisov links in the category of conic bundles. They are used very frequently below. As the first application of the developed theory, following Shokurov [Sho84, in Section 9 we reproduce the proof of Conjecture 1.2 for conic bundles over minimal rational surfaces.

Although Conjectures 1.2 and 1.3 are formulated in terms of nonsingular $X$ and $S$, in order to analyze the corresponding birational maps we use the techniques on the factorization of birational maps between Mori fiber spaces, which, in general, admit terminal singularities. These techniques and results are discussed in Sections 10 13 Thus, Section 10 covers known results on local classification of $\mathbb{Q}$-conic bundles and Section 11 contains numerous examples of Sarkisov links between them. In Sections 12 and 13 we apply developed theory to rationality problems of conic bundles. In particular, prove Sarkisov Theorem 12.2 and Theorem 13.7 which is a very weak version of Conjecture 1.3. We also prove the equivalence of Conjecture 1.3 and some classical conjecture of projective geometry (see Proposition 13.6). In the last section 14 we collect open problems and some results related to birational geometry of conic bundles.

Acknowledgements. The author is grateful to Artem Avilov, Arnaud Beauville, Ivan Cheltsov, Alexander Kuznetsov, Boris Kunyavskii, Constantin Shramov, and Vyacheslav Shokurov for useful advises and discussions, and also to the referees for careful reading the manuscript and numerous corrections of inaccuracies.

\section{Preliminaries}

Everywhere in this paper, if we do not specify anything else, we

work over an algebraically closed field $\mathbb{k}$ of characteristic 0 . In certain 
situations we prefer to work over $\mathbb{C}$. We use the standard terminology and notation of the $(\log )$ Minimal Model Program [KM98]. For an introduction to rationality problems we refer to [KSC04].

2.1. Notation. Throughout this paper

$\equiv$ denotes the numerical equivalence of cycles, $K_{X}$ is the canonical (Weil) divisor of a normal variety $X$, $\mathbb{F}_{n}=\mathbb{P}_{\mathbb{P}^{1}}\left(\mathscr{O}_{\mathbb{P}^{1}} \oplus \mathscr{O}_{\mathbb{P}^{1}}(n)\right)$ is the rational ruled (Hirzebruch) surface,

$\rho(X)=\operatorname{rk} \operatorname{Pic}(X)$ is the Picard number of $X$, $\rho(X / S)$ is the relative Picard number.

A contraction is a surjective projective morphism $\pi: X \rightarrow S$ of normal varieties such that $\pi_{*} \mathscr{O}_{X}=\mathscr{O}_{S}$. A contraction $\pi: X \rightarrow S$ is extremal if $\rho(X / S)=1$.

A contraction is said to be a Mori extremal contraction if $X$ is a $\mathbb{Q}$-factorial variety with at most terminal singularities, the $\mathbb{Q}$-Cartier divisor $-K_{X}$ is relatively ample, and the relative Picard number is $\rho(X / S)=1$. If additionally $\operatorname{dim}(S)<\operatorname{dim}(X)$, then $\pi$ is called a Mori fiber space. In dimension 3 for a Mori fiber space there are only three possibilities listed in 1.1.

Let $\pi: X \rightarrow S$ and $\pi^{\sharp}: X^{\sharp} \rightarrow S^{\sharp}$ be Mori fiber spaces. A birational map $\Phi: X \rightarrow X^{\sharp}$ is said to be fiberwise if there exists a birational map $\alpha: S \rightarrow S^{\sharp}$ making the following diagram

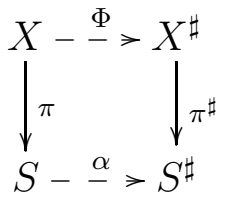

commutative.

For example, $X=\mathbb{P}^{n} \times \mathbb{P}^{n}$ has two Mori fiber space structures $\pi_{i}: X \rightarrow \mathbb{P}^{n}$, the projections to factors. The identity map is not fiberwise birational equivalence but the involution interchanging the factors is.

A Mori fiber space $\pi: X \rightarrow S$ is said to be birationally rigid if given any birational map $\Phi: X \rightarrow X^{\sharp}$ to another Mori fiber space $\pi^{\sharp}: X^{\sharp} \rightarrow S^{\sharp}$, there exists a birational selfmap $\psi: X \rightarrow X$ such that the composition $\Phi \circ \psi: X \rightarrow X^{\sharp}$ is fiberwise:

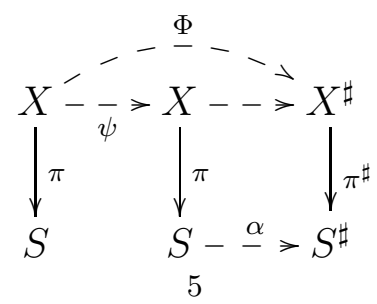


and induces an isomorphism $X_{\eta} \rightarrow X_{\eta}^{\sharp}$ of generic fibers. Examples will be given in Corollary 5.1 .2 and Theorem 12.2. It is important to note that the rigidity implies non-rationality but the rigidity is much stronger.

\section{Conic Bundles}

3.1. Definition. A conic bundle is a proper flat morphism $\pi: X \rightarrow$ $S$ of smooth varieties such that it is of relative dimension one and the anti-canonical divisor $-K_{X}$ is relatively ample. A conic bundle $\pi: X \rightarrow S$ is said to be standard if one of the following equivalent conditions holds:

(a) $\operatorname{Pic}(X)=\pi^{*} \operatorname{Pic}(S) \oplus \mathbb{Z}$,

(b) $\rho(X / S)=1$, i.e. $\pi$ is a Mori extremal contraction,

(c) for any prime divisor $D \subset S$ its preimage $\pi^{*}(D)$ is irreducible.

If $\pi: X \rightarrow S$ is any Mori extremal contraction from a smooth threefold to a surface, then $\pi$ is a standard conic bundle Mor82. There is a similar, but weaker, result in arbitrary dimension: if $X$ is a smooth variety and $\pi: X \rightarrow S$ is a Mori extremal contraction such that the dimension of any fiber equals 1 , then $\pi$ is a standard conic bundle And85.

The following facts are well-known, see [Bea77b, Prop. 1.2], Sar82, $\S 1]$.

3.2. Theorem. Let $\pi: X \rightarrow S$ be a conic bundle. Then we have:

(i) The anti-canonical line bundle $\omega_{X}^{-1}$ is relatively very ample and defines an embedding $X \hookrightarrow \mathbb{P}(\mathscr{E})$, where $\mathscr{E}:=\pi_{*} \omega_{X}^{-1}$ is a locally free sheaf of rank 3.

(ii) In the above embedding $X$ is the zero locus of a section

$$
\sigma \in H^{0}\left(\mathbb{P}(\mathscr{E}), \mathscr{L}^{\otimes 2} \otimes p^{*}\left(\operatorname{det} \mathscr{E}^{\vee} \otimes \omega_{S}^{-1}\right)\right),
$$

where $\mathscr{L}=\mathscr{O}_{\mathbb{P}(\mathscr{E})}(1)$ is the tautological line bundle on $\mathbb{P}(\mathscr{E})$ and $p: \mathbb{P}(\mathscr{E}) \rightarrow S$ is the projection.

(iii) For any point $s \in S$ the scheme-theoretic fiber $X_{s}=\pi^{-1}(s)$ is isomorphic over the residue field $\mathbb{k}(s)$ to a conic (possibly reducible or non-reduced) on the plane $\mathbb{P}(\mathscr{E})_{s}=\mathbb{P}_{\mathbb{k}(s)}^{2}$.

3.3. If $\pi: X \rightarrow S$ is a conic bundle, then a general fiber $X_{s}=\pi^{-1}(s)$ is a non-degenerate conic, i.e. an irreducible smooth rational curve. Put

$$
\begin{aligned}
\Delta & :=\left\{s \in S \mid X_{s} \text { is a degenerate conic }\right\} \\
\Delta_{\mathrm{s}} & :=\left\{s \in S \mid X_{s} \text { is a double line }\right\}
\end{aligned}
$$


Then $\Delta$ is a divisor on $S$. It is called the discriminant (or degeneration) divisor. The set $\Delta_{\mathrm{s}}$ coincides with the singular locus of $\Delta$ (see (3.3.2) below).

In a small affine neighborhood $U \subset S$ of $s \in S$, one can write the equation of $X \subset \mathbb{P}_{x_{0}, x_{1}, x_{2}}^{2} \times U$ in the form

$$
\sum_{0 \leq i, j \leq 2} a_{i, j} x_{i} x_{j}=0
$$

where $a_{i, j} \in \mathbb{C}[U]$. Then $\Delta$ is given by the determinant equation

$$
\operatorname{det}\left\|a_{i, j}(s)\right\|=0 .
$$

3.3.2. Moreover, $\mathrm{rk}\left\|a_{i, j}(s)\right\| \neq 0$ and using $\mathbb{C}[U]$-linear coordinate change one can put the equation (3.3.1) to one of the following forms

$$
\begin{aligned}
b_{0} x_{0}^{2}+b_{1} x_{1}^{2}+b_{2} x_{2}^{2}=0 & \Leftrightarrow \operatorname{rk}\left\|a_{i, j}(s)\right\|=3 \Leftrightarrow s \notin \Delta \\
b_{0} x_{0}^{2}+b_{1} x_{1}^{2}+c_{2} x_{2}^{2}=0 & \Leftrightarrow \operatorname{rk}\left\|a_{i, j}(s)\right\|=2 \Leftrightarrow s \in \Delta \backslash \Delta_{\mathrm{s}} \\
b_{0} x_{0}^{2}+c_{1} x_{1}^{2}+c_{2} x_{2}^{2}+c_{3} x_{1} x_{2}=0 & \Leftrightarrow \operatorname{rk}\left\|a_{i, j}(s)\right\|=1 \Leftrightarrow s \in \Delta_{\mathrm{s}}
\end{aligned}
$$

where $b_{i}(s) \neq 0, c_{i}(s)=0$, and $\operatorname{mult}_{s}\left(c_{1}\right)=\operatorname{mult}_{s}\left(c_{2}\right)=1$.

3.3.3. Corollary ([Sar82, Proof of Proposition 1.8.5]). The discriminant divisor of a conic bundle $\pi: X \rightarrow S$ has only normal crossings in codimension two in $S$.

3.3.4. Remark. Let $\pi: X \rightarrow S$ be a contraction such that there exists a closed subset $Z \subset S$ of codimension $\geq 2$ such that the restriction $\pi^{o}: X^{o} \rightarrow S^{o}$ is a conic bundle, where $S^{o}:=S \backslash Z$ and $X^{o}:=\pi^{-1}\left(S^{o}\right)$. Then we can define the discriminant divisor $\Delta \subset S$ of $\pi$ as the closure of the discriminant curve $\Delta^{o}$ of the conic bundle $\pi^{o}$. In general $\Delta$ is a (reduced) effective Weil divisor. Note however that in this case $\Delta$ is just divisorial part of the maximal subset $R \subset S$ over which $\pi$ is not smooth. We do not assert that $\pi$ is smooth or flat over $S \backslash \Delta$ (see e.g. 10.3.1).

The above definition can be applied to arbitrary Mori extremal contractions $\pi: X \rightarrow S$ such that $\operatorname{dim} X=\operatorname{dim} S+1$ and $X$ has at worst terminal singularities. Indeed, let $S_{\geq 2} \subset S$ be the set of points $s \in S$ such that $\operatorname{dim} \pi^{-1}(s) \geq 2$ and let

$$
Z:=S_{\geq 2} \cup \pi(\operatorname{Sing}(X))
$$

Since $\pi$ is extremal, $\pi^{-1}\left(S_{\geq 2}\right)$ has no divisorial components. Since $X$ has at worst terminal singularities, $\operatorname{codim}_{X} \operatorname{Sing}(X) \geq 3$. Therefore, $\operatorname{codim}_{S} Z \geq 2$. Then $S^{o}:=S \backslash Z$ is smooth and $\pi^{o}$ is a conic bundle over $S^{o}$ (see [Mor82], And85]). 
3.3.5. Lemma. Let $\pi: X \rightarrow S$ and $\pi^{\prime}: X^{\prime} \rightarrow S$ be a standard conic bundles over a (not necessarily proper) variety $S$. Suppose that there is a fiberwise birational equivalence

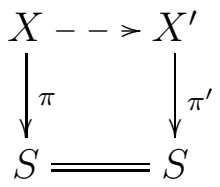

Then discriminant divisors of $\pi$ and $\pi^{\prime}$ coincide.

Proof. Let $\Delta$ and $\Delta^{\prime}$ be discriminant divisors of $\pi$ and $\pi^{\prime}$, respectively. We may assume that $S=\operatorname{Spec} A$ is a small affine neighborhood of a fixed point. Then it is sufficient to consider the case where $\Delta=\varnothing$. Moreover, by shrinking $S$ we may assume that the vector bundles $\pi_{*} \mathscr{O}_{X}\left(-K_{X}\right)$ and $\pi_{*} \mathscr{O}_{X^{\prime}}\left(-K_{X^{\prime}}\right)$ are trivial. Then the anti-canonical divisors define embeddings $X, X^{\prime} \hookrightarrow \mathbb{P}^{2} \times S=\mathbb{P}_{A}^{2}$. Thus $X$ and $X^{\prime}$ are given in $\mathbb{P}_{A}^{2}$ by equations $\sum q_{i, j} x_{i} x_{j}=0$ and $\sum q_{i, j}^{\prime} x_{i} x_{j}=0$, respectively, where $q_{i, j}, q_{i, j}^{\prime} \in A$. By our assumption the matrix $\left\|q_{i, j}\right\|$ is non-degenerate and $\operatorname{det}\left\|q_{i, j}^{\prime}\right\|=0$ is the equation of $\Delta^{\prime}$. Since $X$ and $X^{\prime}$ are birationally equivalent over $S$, the generic fibers $X_{\eta}$ and $X_{\eta}^{\prime}$ are isomorphic over the field $\mathbb{K}:=\operatorname{Frac}(A)$. Hence there exists a projective transformation $\mathbb{P}_{\mathbb{K}}^{2} \rightarrow \mathbb{P}_{\mathbb{K}}^{2}$ that maps $X_{\eta}$ to $X_{\eta}^{\prime}$. Let $T=\left\|t_{i, j}\right\|, t_{i, j} \in \mathbb{K}$ be the corresponding matrix. Then the equation of $\Delta^{\prime}$ is written in the form

$$
\operatorname{det}\left\|q_{i, j}^{\prime}\right\|=\left(\operatorname{det}\left\|t_{i, j}\right\|\right)^{2}\left(\operatorname{det}\left\|q_{i, j}\right\|\right)=0,
$$

where det $\left\|q_{i, j}\right\|$ is an invertible element of $A$. Since the discriminant curve of a standard conic bundle is reduced, the element det $\left\|t_{i, j}\right\|$ must be invertible as well. This means that $\Delta^{\prime}=\varnothing=\Delta$ on $S$.

3.3.6. Corollary. Suppose we have a fiberwise birational equivalence of $\mathbb{Q}$-conic bundles

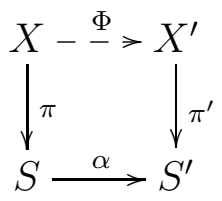

where $\alpha$ is a birational morphism. Then for the corresponding discriminant curves $\Delta \subset S$ and $\Delta^{\prime} \subset S^{\prime}$ one has $\Delta^{\prime}=\alpha(\Delta)$.

The corollary shows that the discriminant curves of fiberwise birational class of $X / S$ define a $b$-divisor on $S$, see Sho03, Isk03. Note however that this is not a b-Cartier b-divisor (if it is not trivial). 
3.4. Lemma. Let $\pi: X \rightarrow S$ be a standard conic bundle. Then there is an isomorphism

$$
\operatorname{Pic}(X) \simeq \begin{cases}\pi^{*} \operatorname{Pic}(S) \oplus \mathbb{Z} \cdot K_{X} & \text { if } \pi \text { has no rational sections, } \\ \pi^{*} \operatorname{Pic}(S) \oplus \mathbb{Z} \cdot D & \text { if } \pi \text { has a rational section } D\end{cases}
$$

From now on, we mainly concentrate on the study of conic bundles over rational surfaces. Let us consider several well-known examples.

3.4.1. Example. Let $X \subset \mathbb{P}^{2} \times \mathbb{P}^{2}$ be a smooth divisor of bidegree $(2, d), d>0$ given by the equation $f\left(x_{0}, x_{1}, x_{2}, y_{0}, y_{1}, y_{2}\right)=0$. This equation is quadratic in $x_{0}, x_{1}, x_{2}$, so it can be viewed as a symmetric $3 \times 3$-matrix $Q$ whose entires are homogeneous polynomials in $y_{0}, y_{1}, y_{2}$ of degree $d$. The projection $X \rightarrow \mathbb{P}^{2}$ to the second factor is a standard conic bundle whose discriminant curve is given by the equation $\operatorname{det}(Q)=0$ of degree $3 d$.

Starting with some well-known rationally connected variety, for example, Fano threefold, in many cases one can construct a conic bundle by using some special birational transformations, so-called Sarkisov links, see Sect. 4. We give several such constructions below.

3.4.2. Example. Let $Y=Y_{3} \subset \mathbb{P}^{4}$ be a smooth cubic hypersurface. It is well known (see e.g. AK77]) that $Y$ contains a two-dimensional family of lines $\Sigma(Y)$. Let $l \subset Y$ be a line and let $\sigma: X \rightarrow Y$ be the blowup of $l$. Let $E$ be the exceptional divisor and let $H^{*}=\sigma^{*} H$ be the pull-back of a hyperplane section. Then the two-dimensional linear system $\left|H^{*}-E\right|$ is base point free and defines a conic bundle structure $\pi: X \rightarrow \mathbb{P}^{2}$. The discriminant curve $\Delta \subset \mathbb{P}^{2}$ is of degree 5. Indeed, a general member $F \in\left|H^{*}-E\right|$ is a cubic surface and the restriction $\left.\pi\right|_{F}$ is a conic bundle over a line $l \subset \mathbb{P}^{2}$. Degenerate fibers of $\left.\pi\right|_{F}$ correspond to points $\Delta \cap l$. By the Noether formula there are exactly 5 such fibers.

For a general choice of $l$ in the corresponding Hilbert scheme, the curve $\Delta$ is smooth. However, for some special choice of $l, \Delta$ can be singular. To illustrate this, we recall that the normal bundle $\mathscr{N}_{\Gamma / Y}$ of any line $\Gamma \subset Y$ has the form $\mathscr{O}_{\mathbb{P}^{1}}(a) \oplus \mathscr{O}_{\mathbb{P}^{1}}(-a)$, where $a=0$ or 1 [KPS18, Proof of Proposition 2.2.8]. The lines with $a=1$ are called special. They are characterized by the property that there exists a plane $\mathbb{P}^{2} \subset \mathbb{P}^{4}$ such that $Y \cap \mathbb{P}^{2}=2 \Gamma+\Gamma^{\prime}$, where $\Gamma^{\prime}$ is also a line which is called complementary to $\Gamma$. The set of complementary lines is a closed one-dimensional subset in $\Sigma(Y)$. Now, if we take in the above construction the line $l=\Gamma^{\prime}$ to be complementary, then the proper transform of $\Gamma$ on $X$ will be a non-reduced fiber of the conic bundle $\pi$ and so the discriminant curve $\Delta$ will be singular at the corresponding 
point. If $l$ is not complementary, then the discriminant curve $\Delta$ is smooth. Moreover, the discriminant curve $\Delta$ can be reducible: suppose that $Y$ contains a cubic cone $Z$. Take a line $l$ so that $l \subset Z$ and let $Z_{X} \subset X$ be the proper transform of $Z$. Then $\Delta_{1}:=\pi\left(Z_{X}\right)$ is an irreducible component of $\Delta \subset \mathbb{P}^{2}$ of degree 1 .

There is another type of conic bundles over $\mathbb{P}^{2}$ with discriminant curve of degree 5 :

3.4.3. Example (cf. [Pan80], BL12]). Let $\Gamma \subset \mathbb{P}^{3}$ be a smooth curve of degree 7 and genus 5. By the Riemann-Roch theorem, the embedding $\Gamma \subset \mathbb{P}^{3}$ is given by the complete linear system $\left|K_{\Gamma}-P\right|$, where $P \in \Gamma$ is a point. Let $\sigma: X \rightarrow \mathbb{P}^{3}$ be the blowup of $\Gamma$ and let $E$ be the exceptional divisor. Let $H^{*}=\sigma^{*} H$ be the pull-back of a hyperplane in $\mathbb{P}^{3}$. Then the linear system $\left|3 H^{*}-E\right|$ is base point free and defines a conic bundle structure $\pi: X \rightarrow \mathbb{P}^{2}$. The fibers of $\pi$ are proper transforms of conics in $\mathbb{P}^{3}$ meeting $\Gamma$ at six points and a general member of $\left|3 H^{*}-E\right|$ is a cubic surface. As above, the discriminant curve $\Delta \subset \mathbb{P}^{2}$ is of degree 5 .

Since $\rho(X)=2$ and both contractions $\pi$ and $\sigma$ are $K_{X}$-negative, $X$ is a Fano threefold with $-K_{X}^{3}=16$ (see [MM82, No. 9]). The morphism $\sigma \times \pi: X \rightarrow \mathbb{P}^{3} \times \mathbb{P}^{2}$ is an embedding and its image is an intersection of two divisors of bidegrees $(1,1)$ and $(2,1)$.

Since any smooth three-dimensional cubic hypersurface is not rational [CG72], these two types of conic bundle (with discriminant curve of degree 5) cannot be birationally equivalent. They differ by the type of the corresponding double cover $\tilde{\pi}: \tilde{\Delta} \rightarrow \Delta$ (see 3.9 below); for a conic bundle originating from a cubic hypersurface, the cover is defined by an odd theta-characteristic, whereas the theta-characteristic for the conic bundle constructed in Example 3.4.3 is even (see 3.9.2 below).

3.4.4. Example ([Bea77b, Example 1.4.4], [IP99, Th. 4.3.3]). Let $Y=Y_{2 \cdot 2 \cdot 2} \subset \mathbb{P}^{6}$ be a smooth complete intersection of three quadrics. It is known that $Y$ contains a one-dimensional family of lines (see e.g. Tyu75, Lemma 5.2]). Let $l \subset Y$ be a line and let $\sigma: \tilde{Y} \rightarrow Y$ be the blowup of $l$. Let $E$ be the exceptional divisor and let $H^{*}=\sigma^{*} H$ be the pull-back of a hyperplane section. The linear system $\left|-K_{\tilde{Y}}\right|$ is base point free and defines a generically finite (but not finite) morphism. For a general choice of line $l$ this morphism is small, i.e. it does not contract divisors. According to [Kol89], there exists a flop $\tilde{Y} \rightarrow X$ and by the Cone Theorem on $X$ there exists a Mori extremal contraction. It is not hard to show that the only possibility for this contraction is conic bundle $\pi: X \rightarrow \mathbb{P}^{2}$ with discriminant curve 
$\Delta \subset \mathbb{P}^{2}$ is of degree 7 . Then the map $\tilde{Y} \rightarrow \mathbb{P}^{2}$ is given by the linear system $\left|2 H^{*}-3 E\right|$, see [IP99, Theorem 4.3.3(ii)] for details.

3.4.5. Example. Let $Y=Y_{2 \cdot 3} \subset \mathbb{P}^{5}$ be an intersection of a quadric and a cubic. Suppose that $Y$ contains a plane $\Pi=\mathbb{P}^{2}$. The projection from $\Pi$ induces a rational curve fibration on $Y$. If $Y$ is sufficiently general, then by blowing up $\Pi$ we obtain a standard conic bundle over $\mathbb{P}^{2}$ with discriminant curve of degree 7, see e.g. [Bea77b, Example 1.4.6]. In this case $Y$ is the midpoint of a Sarkisov link [JPR11, Proposition 7.11].

More examples of conic bundles over $\mathbb{P}^{2}$ with discriminant curve of degree 7 can be found in [BCZ04.

3.4.6. Example ([Bea77b, Example 1.4.3], [CM77a, CM77b]). Let $Y \subset \mathbb{P}^{4}$ be a quartic hypersurface which is singular along a line $l$. Suppose that $Y$ is general. Then by blowing up $l$ we obtain a standard conic bundle over $\mathbb{P}^{2}$ with discriminant curve of degree 8 .

3.4.7. Example ([Bea77b, Example 1.4.5], [Deb90], [CPS15], [Pro17]). Let $\varphi: Y \rightarrow \mathbb{P}^{3}$ be a double cover branched over a quartic surface $B \subset \mathbb{P}^{3}$. Assume that $B$ is singular and its singular locus consists of a unique node $Q$. Then $P:=\varphi^{-1}(Q)$ is a unique singular point of $Y$. In this case, $Y$ is $\mathbb{Q}$-factorial (see e.g. Che09]). Let $\sigma: X \rightarrow Y$ and $\lambda: \tilde{\mathbb{P}}^{3} \rightarrow \mathbb{P}^{3}$ be blowups of $P$ and $Q$, respectively. Then $\varphi$ induces a double cover $\tilde{\varphi}: X \rightarrow \tilde{\mathbb{P}}^{3}$ and the projection from $Q$ induces a $\mathbb{P}^{1}$ bundle $\psi: \tilde{\mathbb{P}}^{3} \rightarrow \mathbb{P}^{2}$. The composition $\pi: X \rightarrow \tilde{\mathbb{P}}^{3} \rightarrow \mathbb{P}^{2}$ is a standard conic bundle whose fibers are double covers of the fibers of $\psi$. The discriminant curve is of degree 6 .

Now if the point $P$ is not a unique singular point of $X$, then in the above construction $X$ is singular and $\pi: X \rightarrow \tilde{\mathbb{P}}^{3} \rightarrow \mathbb{P}^{2}$ is nonstandard conic bundle. The standard forms for $X / \mathbb{P}^{2}$ were described in [CPS15.

3.5. Let $\pi: X \rightarrow S$ be a conic bundle over a surface $S$ and let $\Delta \subset S$ be its discriminant curve. According to discussions in 3.3 the curve $\Delta$ is a reduced normal crossing divisor (possibly $\Delta=\varnothing$ ). Moreover, a fiber $X_{s}, s \in S$ is smooth (resp. a pair of meeting lines, double line) if $s \in S \backslash \Delta($ resp. $s \in \Delta \backslash \operatorname{Sing}(\Delta), s \in \operatorname{Sing}(\Delta))$.

The following facts are easy and well-known.

3.6. Lemma. Let $\pi: X \rightarrow S$ be a conic bundle over a (projective) surface and let $\Delta$ be the discriminant curve. Then

$$
\begin{aligned}
& \chi_{\mathrm{top}}(X)=2 \chi_{\mathrm{top}}(S)-2 \mathrm{p}_{\mathrm{a}}(\Delta)+2 \\
& \mathrm{~b}_{1}(X)=\mathrm{b}_{1}(S) \\
& \mathrm{b}_{3}(X)=2 \mathrm{~b}_{1}(S)+2 \mathrm{~b}_{2}(X)-2 \mathrm{~b}_{2}(S)+2 \mathrm{p}_{\mathrm{a}}(\Delta)-4 \\
& 11
\end{aligned}
$$


(for the case $\Delta=\varnothing$ we put $\mathrm{p}_{\mathrm{a}}(\varnothing)=1$ ).

3.7. Lemma. Let $\pi: X \rightarrow S$ be a conic bundle over a surface and let $\Delta \subset S$ be its discriminant curve.

(i) If $X$ is rationally connected, then the surface $S$ is rational. Conversely, if $\pi: X \rightarrow S$ be a conic bundle over a rational surface, then $X$ is rationally connected KMM92, GHS03.

(ii) If $\pi$ has a rational section, then $X$ is birationally equivalent to $S \times \mathbb{P}^{1}$.

(iii) Assume that the conic bundle is standard and the Brauer group of $S$ is trivial (for example this holds if $S$ is rational). Then $\pi$ has a rational section if and only if $\Delta=\varnothing$.

3.8. Admissible double covers. Let $\tilde{\Delta}$ be a reduced connected curve with at worst nodal singularities and let $\tau: \tilde{\Delta} \rightarrow \tilde{\Delta}$ be an involution (an automorphism of order 2). Let $\Delta:=\tilde{\Delta} / \tau$ be the quotient and let $\tilde{\pi}: \tilde{\Delta} \rightarrow \Delta$ be the natural projection. It is easy to show that the singularities of $\Delta$ are also at worst nodes. We also assume that the following condition is satisfied (the so-called Beauville condition):

$$
\pi(\operatorname{Sing}(\tilde{\Delta}))=\operatorname{Sing}(\Delta), \quad \operatorname{Sing}(\tilde{\Delta})=\{x \in \tilde{\Delta} \mid \tau(x)=x\} .
$$

Then the restriction of $\tilde{\pi}$ to each irreducible component of $\tilde{\Delta}$ does not split and, at a singular point of $\tilde{\Delta}$, two branches are not switched by $\tau$. From (3.8.1) one can easily deduce the following condition:

for every decomposition $\Delta=\Delta_{1}+\Delta_{2}$ with $\Delta_{i} \geq 0$ we have $\#\left(\Delta_{1} \cap \Delta_{2}\right) \equiv 0 \bmod 2$.

Note however, that in our situation $\tilde{\pi}$ is not necessarily flat.

3.9. Now, let $\pi: X \rightarrow S$ be a standard conic bundle over a projective surface $S$ and let $\Delta \subset S$ be its discriminant curve. Let $\tilde{\Delta}$ be the curve parameterizing components of fibers in the ruled surface $X_{\Delta}:=$ $\pi^{-1}(\Delta)$. The induced projection $\tilde{\pi}: \tilde{\Delta} \rightarrow \Delta$ is finite of degree 2 and satisfies the conditions of 3.8 .

3.9.1. Corollary. Any connected component of the discriminant divisor of a standard conic bundle over a surface has arithmetic genus at least 1 .

3.9.2. Recall that a theta-characteristic of a smooth curve $\Delta$ is the linear equivalence class of a divisor $D$ such that $2 D \sim K_{\Delta}$.

A theta-characteristic can be even or odd depending on the parity of the dimension of $H^{0}\left(\Delta, \mathscr{O}_{\Delta}(D)\right.$ ) (see e.g. [Mum71, [Dol12, Ch. 5]). This definition can be naturally generalized to the case of reduced Gorenstein curves. A theta-characteristic of such a curve is a rank-1 torsion-free sheaf $\mathscr{F}$ such that $\mathscr{H} \operatorname{om}_{\mathscr{O}}\left(\mathscr{F}, \omega_{\Delta}\right) \simeq \mathscr{F}$ [Bea77a], [Pio07]. 
3.9.3. Example. Suppose that $S=\mathbb{P}^{2}, \Delta$ is smooth, and the degree of $\Delta$ is odd and $\geq 5$. Write $\operatorname{deg} \Delta=2 m+3$. There is one-to-one correspondence between étale double covers $\tilde{\pi}: \tilde{\Delta} \rightarrow \Delta$ and elements $\sigma$ of order 2 in the Jacobian $\mathrm{J}(\Delta)$. The linear system $|m h|$, where $h$ is the class of hyperplane section of $\Delta$, is a half-canonical linear system, i.e. it is a theta-characteristic. Then $m h+\sigma$ is another theta-characteristic. In other words, the group of 2-torsion points $\mathrm{J}_{2}(\Delta) \subset \mathrm{J}(\Delta)$ acts on the set of all theta-characteristics $\operatorname{Th}(\Delta)$ making it a principal homogeneous space. The choice of a distinguished point $|m h| \in \operatorname{Th}(\Delta)$ establishes an identification $\operatorname{Th}(\Delta) \simeq \mathrm{J}_{2}(\Delta)$. We say that $m h+\sigma$ is the theta-characteristic corresponding to the cover $\tilde{\Delta} \rightarrow \Delta$. Thus there is one-to-one correspondence between étale double covers $\tilde{\pi}: \tilde{\Delta} \rightarrow \Delta$ and theta-characteristics of $\Delta$.

It turns out that the cover $\tilde{\Delta} \rightarrow \Delta$ is the most important invariant. In particular, this cover "almost determine" the conic bundle:

3.10. Proposition. Let $S$ be a rational surface, $\Delta \subset S$ a reduced normal crossing curve, and $\tilde{\pi}: \tilde{\Delta} \rightarrow \Delta$ a double cover satisfying conditions (3.8.1). Then there exists a standard conic bundle $\pi: X \rightarrow S$ with the given $\tilde{\pi}: \tilde{\Delta} \rightarrow \Delta$, and all such standard conic bundles are birationally equivalent over $S$.

Sketch of the proof. Consider so-called Artin-Mumford exact sequence $0 \rightarrow \operatorname{Br} S \rightarrow \operatorname{Br} \mathbb{k}(S) \stackrel{\alpha}{\longrightarrow} \bigoplus_{\substack{\text { curves } \\ C \subset S}} H_{\mathrm{et}}^{1}(C, \mathbb{Q} / \mathbb{Z}) \stackrel{\beta}{\longrightarrow} \bigoplus_{\substack{\text { points } \\ P \in C}} \boldsymbol{\mu}^{-1} \stackrel{\gamma}{\rightarrow} \boldsymbol{\mu}^{-1} \rightarrow 0$ (see [AM72]), where

$$
\boldsymbol{\mu}^{-1}=\cup_{n} \operatorname{Hom}\left(\boldsymbol{\mu}_{n}, \mathbb{Q} / \mathbb{Z}\right),
$$

and $\boldsymbol{\mu}_{n}$ denotes the group of $n$-th roots of unity. Then the double cover $\tilde{\pi}: \tilde{\Delta} \rightarrow \Delta$ determines an element

$$
a \in \bigoplus_{C \subset \Delta} H_{\text {èt }}^{1}(C, \mathbb{Q} / \mathbb{Z})
$$

of order 2 (a collection of local invariants).

From (3.8.1) it follows immediately that $\beta(a)=0$, and hence there is an element $A \in \operatorname{Br}(\mathbb{k}(S))$ such that $\alpha(A)=a$. As $S$ is a rational surface, we have $\operatorname{Br}(S)=0$. Hence, $A \in \operatorname{Br}(\mathbb{k}(S))$ has order 2. By virtue of the well-known Merkur'ev theorem Mer81] $A$ is a product of classes of quaternion algebras over the function field $\mathbb{k}(S)$. Furthermore, since $\mathbb{k}(S)$ is a $\mathrm{c}_{2}$-field, by Albert's theorem [Jac96, Theorem 2.10 .9 the product of classes of quaternion algebras is also represented by a quaternion algebra. Thus there exists a quaternion algebra $\mathscr{A}$ 
over $\mathbb{k}(S)$ whose class in $\operatorname{Br}(\mathbb{k}(S))$ is equal to $A$. According to the classical theorem on central simple algebras $\mathscr{A}$ is uniquely determined up to isomorphism. As in AM72] (see also [Sar82, Theorem 5.3]), one proves that the maximal orders of $\mathscr{A}$ over $S$ are in 1-1 correspondence with standard conic bundles $\pi: X \rightarrow S$ with given local invariants $\tilde{\pi}: \tilde{\Delta} \rightarrow \Delta$ (3.10.1). All such conic bundles are birationally equivalent over $S$ since their generic fibers are isomorphic conics over $\mathbb{k}(S)$ associated to the same quaternion algebra $\mathscr{A}$.

3.10.2. Example. Let $X \subset \mathbb{P}_{u_{0}, u_{1}, u_{1}}^{2} \times \mathbb{P}_{x_{0}, x_{1}, x_{1}}^{2}$ be given by the equation

$$
f_{0}\left(u_{0}, u_{1}, u_{1}\right) x_{0}^{2}+f_{1}\left(u_{0}, u_{1}, u_{1}\right) x_{1}^{2}+f_{2}\left(u_{0}, u_{1}, u_{1}\right) x_{2}^{2}=0
$$

where $f_{i}$ are general homogeneous polynomials of degree $d \geq 1$. The projection $\pi: X \rightarrow \mathbb{P}^{2}$ to the first factor is a standard conic bundle whose discriminant curve is reducible curve of degree $3 d$ given by $f_{0} f_{1} f_{2}=0$. The corresponding quaternion algebra $A$ over $\mathbb{k}\left(\mathbb{P}^{2}\right)$ is generated by four vectors $\mathbf{1}, \mathbf{i}, \mathbf{j}$, $\mathbf{k}$ with standard relations

$$
\mathbf{i} \cdot \mathbf{j}=\mathbf{k}=-\mathbf{j} \cdot \mathbf{i}, \quad \mathbf{i}^{2}=-f_{0} / f_{2}, \quad \mathbf{j}^{2}=-f_{1} / f_{2}, \quad \mathbf{k}^{2}=-f_{0} f_{1} / f_{2}^{2} .
$$

Note however that the recipe given in Proposition 3.10 allows to recover a conic bundle $\pi: X \rightarrow S$ only up to birational equivalence over the base $S$. There is no canonical way of reconstruction of $X / S$ by the double cover $\tilde{\Delta} \rightarrow \Delta$.

3.11. Lemma (cf. [Sar81]). Let $\pi: X \rightarrow S$ be a contraction of projective varieties such that any fiber is one-dimensional, $X$ has at worst terminal singularities, and $-K_{X}$ is $\pi$-ample. Let $\Delta \subset S$ be the discriminant divisor. Then the following numerical equivalence of cycles on $S$ holds, where $K_{X}^{2}$ is regarded as a rational equivalence class of codimension two cycles

$$
-\pi_{*} K_{X}^{2} \equiv 4 K_{S}+\Delta
$$

Proof. We prove (3.11.1) by induction on the dimension. If $\operatorname{dim} X=2$, then $X$ is smooth and (3.11.1) is an immediate consequence of the Noether formula. For $\operatorname{dim} X \geq 3$, let $Z \subset S$ be an effective very ample divisor and let $Y:=\pi^{-1}(Z)$. Take $Z$ to be general in the corresponding linear equivalence class. Then $\pi_{Y}: Y \rightarrow Z$ will satisfy the same conditions as $\pi: X \rightarrow S$. Let $\Delta_{Z}=\Delta \cap Z$ be the corresponding discriminant locus. It is sufficient to show that

$$
\left(\pi_{*} K_{X}^{2}+4 K_{S}+\Delta\right) \cdot Z \equiv 0 .
$$

Using the projection and adjunction formulas we can write

$$
\begin{aligned}
& \left(\pi_{*} K_{X}^{2}+4 K_{S}+\Delta\right) \cdot Z \equiv \pi_{*}\left(K_{X}^{2} \cdot Y\right)+4\left(K_{Z}-Z^{2}\right)+\Delta_{Z} \equiv \\
& \equiv \pi_{*} K_{Y}^{2}-2 \pi_{*}\left(K_{Y} \cdot Y^{2}\right)+4 K_{Z}-4 Z^{2}+\Delta_{Z} \equiv \pi_{*} K_{Y}^{2}+4 K_{Z}+\Delta_{Z} .
\end{aligned}
$$


By the inductive hypothesis this proves (3.11.1).

3.12. Theorem. Given any $\mathbb{Q}$-conic bundle $\pi: X \rightarrow S$, there is a birational contraction $\alpha: S^{\bullet} \rightarrow S$ and a standard conic bundle $\pi^{\bullet}$ : $X \rightarrow S^{\bullet}$ that is fiberwise birationally equivalent to $\pi: X \rightarrow S$. More precisely, there exists a commutative diagram

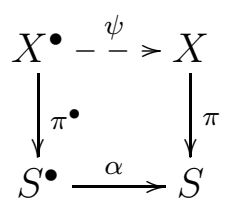

where $\psi$ is a birational map and $\alpha$ is a birational morphism.

This theorem is a particular case of more general fact proved by Sarkisov: for any dominant rational map $g: Y \rightarrow T$ of relative dimension 1 whose generic fiber is an irreducible rational curve there exists a standard conic bundle $\pi^{\bullet}: X \rightarrow S^{\bullet}$ that is fiberwise birationally equivalent to $g$. The proof can be found in Sar82, Theorem 1.13]. Three-dimensional case was outlined earlier in [Zag77] and [Miy83]. See also Avi14 for the three-dimensional equivariant version.

\section{SARKISOV CATEGORY}

In this section we describe the general structure of Sarkisov program. For details we refer to [Cor95, see also [Mat02, Ch. 13], [Isk01], [IS05], [SC11, [HM13.

4.1. First, we recall the definition of a Sarkisov link Cor95, Definition 3.4]. Suppose that $\pi: X \rightarrow S$ and $\pi_{1}: X_{1} \rightarrow S_{1}$ are Mori fiber spaces. A Sarkisov link $\Phi: X_{1} \rightarrow X$ between them is a transformation of one of four types
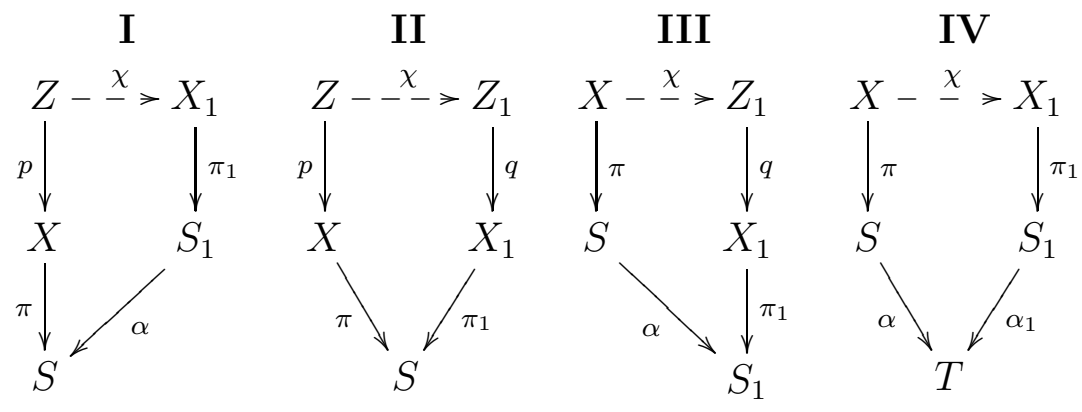

where $p: Z \rightarrow X$ and $q: Z_{1} \rightarrow X_{1}$ are divisorial Mori extremal contractions, $\chi$ is a finite sequence of log-flips, in particular, $\chi$ is an isomorphism in codimension one, and $\alpha$ and $\alpha_{1}$ are extremal contractions in the log terminal category. 
A link of type III is a birational transformation that is inverse to a transformation corresponding to a type $\mathbf{I}$ link. For standard conic bundles, links of types I and III are exactly elementary transformations described in Proposition 8.2. In dimension $\leq 3$, for a type IV link the contractions $S \rightarrow T$ and $S_{1} \rightarrow T$ must be of fiber type (so the link switches the Mori fiber space structures). In higher dimensions the contractions $S \rightarrow T$ and $S_{1} \rightarrow T$ can be small.

4.2. Theorem. Let $\pi: X \rightarrow S$ and $\pi^{\sharp}: X^{\sharp} \rightarrow S^{\sharp}$ be Mori fiber spaces and let

$$
\Phi: X \rightarrow X^{\sharp} \text {. }
$$

be a (not necessarily fiberwise) birational map. Then $\Phi$ is a composition of Sarkisov links.

The three-dimensional version of this program was outlined in preprints [Sar87], SSar89], [Rei91] and a complete proof (with termination) was given in Cor95] (see also [IS05], [SC11]). In higher dimensions the program is established in a weaker form HM13. This approach uses a little different decomposition algorithm. We follow the "standard" variant Cor95 which is more suitable for our applications. The process of decomposition which existence is claimed in Theorem 4.2 is called the Sarkisov program. Below we outline the general structure of this program.

4.3. Let $\mathscr{H}^{\sharp}$ be a very ample linear system on $X^{\sharp}$ and let $\mathscr{H}$ be its proper transform on $X$. Clearly, we can write

$$
\begin{aligned}
\mathscr{H}^{\sharp} & \equiv-\mu^{\sharp} K_{X}+\pi^{\sharp *} A^{\sharp}, \\
\mathscr{H} & \equiv-\mu K_{X}+\pi^{*} A,
\end{aligned}
$$

where $\mu^{\sharp}$ and $\mu$ are positive rational numbers and $A^{\sharp}$ (resp. $A$ ) is a $\mathbb{Q}$-divisor on $S^{\sharp}$ (resp. on $S$ ). We say that the pair $(X, \mathscr{H})$ or the linear system $\mathscr{H}$ has maximal singularity if the pair $\left(X, \frac{1}{\mu} \mathscr{H}\right)$ is not canonical.

The basic tool of the method of maximal singularities is the NoetherFano inequality. Below is its version which is adapted for Sarkisov program.

4.4. Theorem (Noether-Fano inequalities [Cor95, Theorem 4.2], [Isk04, Puk13, Ch. 1, Def. 1.4]). In the above notation the following hold.

(i) $\mu \geq \mu^{\sharp}$ and equality implies that $\Phi$ is fiberwise and induces an isomorphism $\Phi_{\eta}: X_{\eta} \rightarrow X_{\eta}^{\sharp}$ of generic fibers. 
(ii) If $\left(X, \frac{1}{\mu} \mathscr{H}\right)$ is canonical and $K_{X}+\frac{1}{\mu} \mathscr{H}$ is nef, then $\Phi$ is an isomorphism, and it also induces an isomorphism $S \simeq S^{\sharp}$. In particular, $\mu=\mu^{\sharp}$.

4.5. Case where $\mathscr{H}$ has maximal singularity. This step is often called "untwisting maximal singularities". Since $X$ is terminal, the pair $(X, c \mathscr{H})$ is canonical but not terminal for some $0<c<\frac{1}{\mu}$. This number $c$ is called the canonical threshold of $(X, \mathscr{H})$ and denoted by $\mathrm{c}(X, \mathscr{H})$. There exists a log-crepant extremal blowup $p:\left(Z, c \mathscr{H}_{Z}\right) \rightarrow$ $(X, c \mathscr{H})$. Thus we have $\rho(Z / S)=2$ and

$$
K_{Z}+c \mathscr{H}_{Z} \equiv p^{*}\left(K_{X}+c \mathscr{H}\right) .
$$

Moreover, $p$ is a divisorial contraction, $Z$ is $\mathbb{Q}$-factorial and has terminal singularities. Then we run the $\left(K_{Z}+c \mathscr{H}_{Z}\right)$-MMP over $S$. Since $\rho(Z / S)=2$ we obtain one of the links I or II. Then we replace $X$ with $X_{1}$ and $\mathscr{H}$ with its proper transform, and continue the process.

4.5.1. Each step as above either decreases the number e $(X, c \mathscr{H})$ of crepant divisors or (if e $(X, c \mathscr{H})=1$ ) increases the canonical threshold $\mathrm{c}(X, \mathscr{H})$. The number $\mathrm{e}(X, c \mathscr{H})$ is a positive integer, so it cannot decrease infinitely many times. Termination of an increasing sequence of thresholds $\mathrm{c}(X, \mathscr{H})$ is more delicate problem. At the moment it is not known that the set of all canonical thresholds satisfy ascending chain condition (ACC) even in dimension 3. Particular cases of this problem were studied in [Pro08], [Ste11], and [Shr06a]. Termination of the Sarkisov program in [Cor95, Theorem 6.1] was proved by reduction to the log canonical case [Ale94a], [HMX14]. The approach of [HM13] is different (see also [IS05], [SC11]).

\subsection{Case where $\mathscr{H}$ has no maximal singularities. By (4.3.2)}

$$
\mathscr{H}+\mu K_{X} \equiv \pi^{*} A,
$$

where the divisor $A$ is not nef by Theorem 4.4)(ii). If $\rho(S) \leq 1$, then $-\left(\mathscr{H}+\mu K_{X}\right)$ is ample and we just run the $\left(\bar{K}_{X}+\frac{1}{\mu} \mathscr{H}\right)-\mathrm{MMP}$ and get a link of type $\mathbf{I V}$.

Assume that $\rho(S)>1$. In particular, this implies that $S$ is a surface. There is a natural identification of Mori cones $\overline{\mathrm{NE}}(S)=\pi_{*} \overline{\mathrm{NE}}(X)$. By the cone theorem on $X$, the cone $\overline{\mathrm{NE}}(S) \cap\{z \mid A \cdot z<0\}$ is locally polyhedral. Hence there exist extremal rays $R_{S} \subset \overline{\mathrm{NE}}(S)$ and $R_{X} \subset \overline{\mathrm{NE}}(X)$ such that $\left(\mathscr{H}+\mu K_{X}\right) \cdot R_{X}<0, A \cdot R_{S}<0$, and $\pi_{*} R_{X}=R_{S}$. Since $\rho(S)>1$, we have $R_{S}^{2} \leq 0$ and one can show that $R_{S}$ is contractible. Thus there exists its contraction $S \rightarrow T$ with $\rho(S / T)=1$ and $\operatorname{dim} T=2$ or 1 . Then we run the $\left(K_{X}+\frac{1}{\mu} \mathscr{H}\right)$-MMP 
over $T$. This produces a link of type III with $S_{1}=T$ or a link of type IV.

In all these cases, for links of type III or IV, we get a new Mori fiber space $\pi_{1}: X_{1} \rightarrow S_{1}$ such that

$$
\mu_{1} \leq \mu
$$

where $\mu_{1}$ is defined for the proper transform $\mathscr{H}_{X_{1}}$ of $\mathscr{H}$ similar to (4.3.1) and (4.3.2). Since the numbers $\mu$ have bounded denominators (see [Kaw92]), this shows that the sequence of these links also terminates.

\section{SurfaCES OVER NON-CLOSED FIELDS}

In this section all the varieties (surfaces and curves) are supposed to be defined over a perfect field $\mathbb{k}$ of arbitrary characteristic. If $\pi$ : $X \rightarrow B$ is a two-dimensional conic bundle over a curve $B$, then, as in 3.1 , we say that it is standard if $\rho(X / B)=1$. The discriminant locus in this case is a (reduced) zero-dimensional subscheme $\Delta \subset B$ (or empty). Every degenerate geomtric fiber $X_{\bar{b}}, \bar{b} \in B \otimes \overline{\mathbb{K}}$ is a union of two (-1)-curves meeting transveresely at one point (see Fig. 11). If the surface $X$ is geometrically rational, then by the Noether formula one has

$$
K_{X}^{2}+\operatorname{deg} \Delta=8
$$

In particular, $K_{X}^{2} \leq 8$ and the equality $K_{X}^{2}=8$ implies that $\pi$ is a smooth morphism. It is easy to show that for a standard conic bundle $K_{X}^{2} \neq 7$ (see e.g. [Isk80b]).

The Sarkisov program works in the category of surfaces over $\mathbb{k}$. Moreover, in the two-dimensional case all the dashed arrows (log flips) must be isomorphisms. So, the Sarkisov links have the following simple

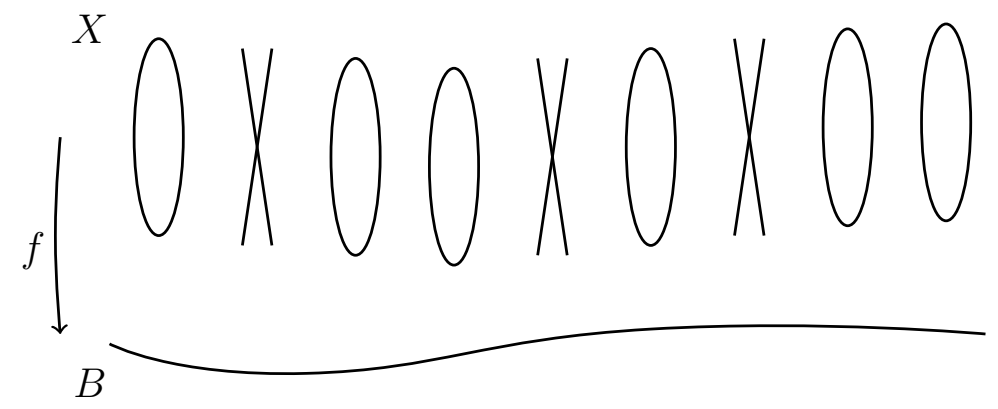

FIGURE 1. 
form:
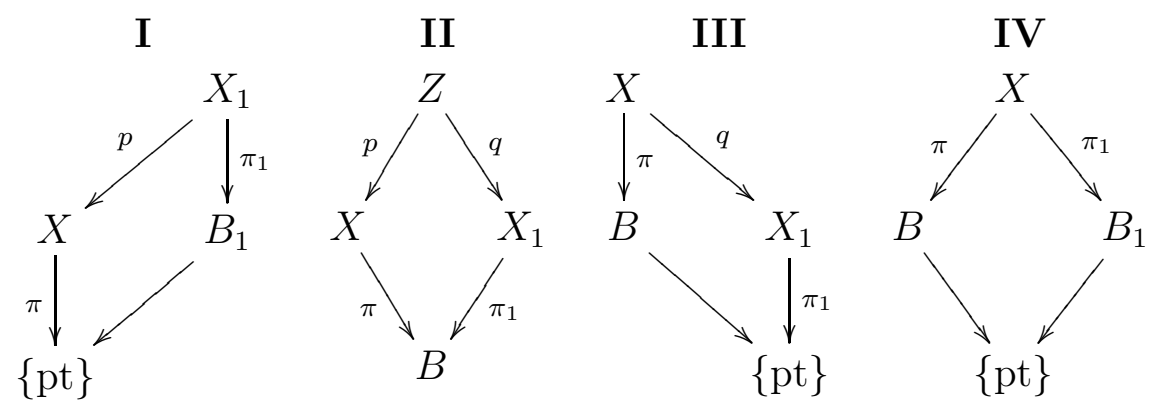

In the case II we distinguish two subcases:

$\mathbf{I I}_{\mathbf{0}} B$ is a point,

II $_{\mathbf{1}} B$ is a curve.

Here is a short description of the links. In all cases morphisms $p$ and $q$ are blowups of closed points.

\begin{tabular}{l|l|l} 
type & $X$ and $\pi$ & $X_{1}$ and $\pi_{1}$ \\
\hline I & $\begin{array}{l}\text { del Pezzo surface with } \\
\rho(X)=1\end{array}$ & $\begin{array}{l}X_{1} \text { is a del Pezzo surface with } \\
\rho\left(X_{1}\right)=2 \\
\pi_{1} \text { is a conic bundle }\end{array}$ \\
\hline $\mathbf{I I}_{\mathbf{0}}$ & $\begin{array}{l}\text { del Pezzo surface with } \\
\rho(X)=1\end{array}$ & $\begin{array}{l}\text { del Pezzo surface with } \\
\rho\left(X_{1}\right)=1\end{array}$ \\
& \multicolumn{1}{|c}{$Z$ is a del Pezzo surface with $\rho(Z)=2$} \\
\hline $\mathbf{I I}_{\mathbf{1}}$ & standard conic bundle & standard conic bundle \\
\hline III & $\begin{array}{l}X \text { is a del Pezzo surface with } \\
\rho(X)=2\end{array}$ & $\begin{array}{l}\text { del Pezzo surface with } \\
\rho\left(X_{1}\right)=1\end{array}$ \\
& $\pi$ is a conic bundle & standard conic bundle \\
\hline IV & \multicolumn{2}{|c}{$X$ istandard conic bundle a del Pezzo surface with $\rho(X)=2$}
\end{tabular}

Iskovskikh [Isk96a classified all links and relations between them in the category of surfaces over non-closed fields. Below we reproduce a part of this classification.

5.1. Theorem ([Isk96a, Theorem 2.6], [Cor95, Theorem A.4], [Pro15b]). Let $X / B \rightarrow X_{1} / B_{1}$ be a Sarkisov link in the category of rational surfaces. Assume that $K_{X}^{2} \leq 4$. Then one of the following holds.

(i) Type $\mathbf{I}$ : $K_{X}^{2}=4, K_{X_{1}}^{2}=3, X_{1}$ is a cubic surface containing a line $l$ defined over $\mathbb{k}, p$ is a contraction of $l, \pi_{1}$ is a projection from $l$. 
(ii) Type II: $B$ is a point, $K_{X}^{2}=2, K_{Z}^{2}=1, p$ and $q$ are blowups of points of degree 1, the link is represented by the Bertini involution on $Z$.

(iii) Type II: $B$ is a point, $K_{X}^{2}=3, K_{Z}^{2}=1, p$ and $q$ are blowups of points of degree 2 , the link is represented by the Bertini involution on $Z$.

(iv) Type II: $B$ is a point, $K_{X}^{2}=3, K_{Z}^{2}=2, p$ and $q$ are blowups of points of degree 1, the link is represented by the Geiser involution on $Z$.

(v) Type II: $B$ is a curve, the link consists of elementary transformations in non-degenerate geometric fibers which are conjugate over $\mathbb{k}$.

(vi) Type III: inverse to (i).

(vii) Type IV: $K_{X}^{2}=1$, the link is represented by the (biregular) Bertini involution on $X$.

(viii) Type IV: $K_{X}^{2}=2$, the link is represented by the (biregular) Geiser involution on $X$.

(ix) Type IV: $K_{X}^{2}=4, X=X_{4} \subset \mathbb{P}^{4}$ is an intersection of two quadrics containing two pencils of conics. Typically, this link is not represented by a biregular involution.

5.1.1. Corollary (โsk67], [sk70], [Isk72], [Isk96a]). Let $\pi: X \rightarrow B$ and $\pi^{\prime}: X^{\prime} \rightarrow B^{\prime}$ be standard conic bundles over rational curves and let $\Phi: X \rightarrow X^{\prime}$ be a birational map.

(i) If $K_{X}^{2} \leq 0$, then $\Phi$ is fiberwise birational.

(ii) If $K_{X}^{2}=1,2$, or 3 , then there exists a birational automorphism $\psi: X \rightarrow X$ such that $\Phi \circ \psi$ is fiberwise birational.

5.1.2. Corollary. Let $\pi: X \rightarrow B$ be a standard conic bundle over a rational curve.

(i) If $K_{X}^{2} \leq 3$, then $\pi: X \rightarrow B$ is birationally rigid.

(ii) If $K_{X}^{2} \leq 0$, then $\pi: X \rightarrow B$ is birationally superrigid.

(iii) If $K_{X}^{2}=4$, then $X$ is not $\mathbb{k}$-rational.

Note that the condition $K_{X}^{2} \leq 0$ is equivalent to $\left|4 K_{B}+\Delta\right| \neq \varnothing$ (cf. Theorem 12.2).

It turns out that a surface with a structure of standard conic bundle and positive self-intersection of the canonical class is "almost" a del Pezzo surface with a few exceptions:

5.2. Proposition ([KM17, Lemma 17], see also [Isk70, [Isk72, [Isk80b], [Pro15b, §8]). Let $\pi: X \rightarrow B$ be a surface standard conic bundle with $0<K_{X}^{2}<8$ and let $\Lambda$ be a geometric fiber. Then the divisor $-K_{X}$ is nef and big except for the following two cases 
(i) $K_{X}^{2}=1$ and there exists a geometrically irreducible rational curve $C \in\left|-K_{X}-\Lambda\right|$ such that $C^{2}=-3$,

(ii) $K_{X}^{2}=2$ and there exists a curve $C \in\left|-K_{X}-2 \Lambda\right|$ which is a pair of conjugate disjoint sections $C_{1}$ and $C_{2}$ with $C_{i}^{2}=-3$.

If furthermore $K_{X}^{2}>2$ and $K_{X}^{2} \neq 4$, then $-K_{X}$ is ample with one exception:

(iii) $K_{X}^{2}=4$ and there exists a curve $C \in\left|-K_{X}-2 \Lambda\right|$ which is a pair of conjugate disjoint sections $C_{1}$ and $C_{2}$ with $C_{i}^{2}=-2$.

In the case (iii) contracting the sections $C_{1}$ and $C_{2}$ we obtain a singular del Pezzo surface of degree 4. Its anticanonical image is (quite special) intersection of two quadrics in $\mathbb{P}^{4}$. This surface is called the Iskovskikh surface [KST89, [CT88.

Proof. Put $d:=K_{X}^{2}$. By the Riemann-Roch formula $\operatorname{dim}\left|-K_{X}\right| \geq$ $d>0$.

Assume that $K_{X} \cdot C>0$ for some reduced irreducible curve $C$. Then $C$ is a fixed component of $\left|-K_{X}\right|$. Hence, $C \sim-K_{X}-\beta \Lambda$ and so $C \cdot \Lambda=2$. In particular, this implies that $C \otimes \overline{\mathbb{k}}$ has at most two components. We have

$$
0>-K_{X} \cdot C=d-2 \beta, \quad 2 \beta>d .
$$

If $C \otimes \overline{\mathbb{K}}$ is connected, then

$$
-2 \leq 2 \mathrm{p}_{\mathrm{a}}(C)-2=\left(K_{X}+C\right) \cdot C=-2 \beta, \quad \beta=1, \quad \mathrm{p}_{\mathrm{a}}(C)=0 .
$$

In this case, $d=1$ and $C^{2}=-3$. We obtain the case (i).

Assume that $C \otimes \overline{\mathbb{k}}$ has two connected components $C_{1}$ and $C_{2}$. In this case, $\mathrm{p}_{\mathrm{a}}\left(C_{i}\right)=0, C_{i}^{2}=\frac{1}{2} d-2 \beta$, and $-K_{X} \cdot C_{i}=\frac{1}{2} d-\beta$. In particular, $d$ is even and $\beta \geq \frac{1}{2} d$. Then

$$
-2=2 \mathrm{p}_{\mathrm{a}}\left(C_{1}\right)-2=\left(K_{X}+C_{1}\right) \cdot C_{1}=-\beta .
$$

Thus $\beta=2=d$. We obtain the case (ii).

Now we consider the case where $-K_{X}$ is nef (but not ample). Let $C$ be an irreducible curve such that $K_{X} \cdot C=0$. Write $C \sim \alpha\left(-K_{X}\right)-\beta \Lambda$ and let $C^{(1)}, \ldots, C^{(k)}$ be connected components of $C \otimes \overline{\mathbb{k}}$. Since every component $C^{(i)}$ is a tree of smooth rational curves, we have $\left(C^{(i)}\right)^{2}=$ -2 and $C^{2}=-2 k$. Further,

$$
0=-K_{X} \cdot C=d \alpha-2 \beta, \quad-2 k=C^{2}=\alpha(d \alpha-4 \beta) .
$$

This gives us $d \alpha=2 \beta,-2 k=\alpha(d \alpha-4 \beta)$, and $k=\alpha \beta$. Let $m:=$ $C^{(i)} \cdot \Lambda$. Then $m k=C \cdot \Lambda=2 \alpha$. Hence, $m \beta=2$ and $d \alpha=4 / m$. Since $d>2$ by our assumptions, the only possibility is $d=4, m=$ $\alpha=1, \beta=2=k$. Since $m=1$, each connected component $C^{(i)}$ is (geometrically) irreducible. We obtain the case (iii) 
Thus in the cases $K_{X}^{2}=3,5,6$ there exists an $K_{X}$-negative extremal ray on $X$ whose contraction $q: X \rightarrow X^{\prime}$ is different from $\pi$ [Mor82]. It is not hard to to find out all the possibilities for $q$ :

5.3. Theorem (【sk80b], [sk96a]). Let $\pi: X \rightarrow B$ be a standard conic bundle, where $B$ is a curve. If $K_{X}^{2} \in\{3,5,6\}$, then there exists a birational contraction $q: X \rightarrow X^{\prime}$, where $X^{\prime}$ is a del Pezzo surface with $\rho\left(X^{\prime}\right)=1$. More precisely, one of the following holds.

(i) If $K_{X}^{2}=3$, then $K_{X^{\prime}}^{2}=4$ and $q$ is a contraction of a geometrically irreducible $(-1)$-curve. In this case $B \simeq \mathbb{P}^{1}$ and the contraction $q: X \rightarrow X^{\prime} \subset \mathbb{P}^{4}$ is given by the linear system $\left|-2 K_{X}-C\right|$, where $C$ is the class of a fiber.

(ii) If $K_{X}^{2}=5$, then $X^{\prime} \simeq \mathbb{P}^{2}$ and $q$ is a contraction of four conjugate $(-1)$-curves. In this case $B \simeq \mathbb{P}^{1}$ and the contraction $q: X \rightarrow \mathbb{P}^{2}$ is given by $\left|-K_{X}-C\right|$, where $C$ is the class of a fiber.

(iii) If $K_{X}^{2}=6$, then $K_{X^{\prime}}^{2}=8$ and $q$ is a contraction of a pair of conjugate $(-1)$-curves. The contraction $q: X \rightarrow X^{\prime} \subset \mathbb{P}^{8}$ is given by $\left|-2 K_{X}+\pi^{*} K_{B}\right|$.

5.4. Corollary. Let $\pi: X \rightarrow B$ be a standard conic bundle, where $X$ is a geometrically rational surface, and $B$ is a curve. Let $\Delta \subset B$ be the discriminant locus.

(i) $\operatorname{deg} \Delta \neq 1$.

(ii) If $\operatorname{deg} \Delta=3$, then $X$ is $\mathbb{k}$-rational.

(iii) If $X$ has a $\mathbb{k}$-point and either $\operatorname{deg} \Delta=2$ or $\Delta=\varnothing$, then $X$ is $\mathbb{k}$-rational.

Putting the above results together one obtains the following criterion of rationality.

5.5. Theorem (see [Isk96a, Theorem 2.6]). Let $X$ be a minimal rational surface over $\mathbb{k}$. Then $X$ is $\mathbb{k}$-rational if and only if $K_{X}^{2} \geq 5$ and $X$ has a $\mathbb{k}$-point.

As a consequence of Corollary 5.4 we have the following.

5.6. Proposition. Let the ground field $\mathbb{k}$ be algebraically closed. Let $\pi: X \rightarrow S$ be a conic bundle over a rational surface with discriminant curve $\Delta \subset S$. Assume that there exists a base point free pencil $\mathscr{L}$ of rational curves on $S$ such that $\mathscr{L} \cdot \Delta \leq 3$. Then $X$ is rational.

Proof. Consider the generic member $L_{\eta}$ of $\mathscr{L}$ over the residue field $\mathbb{k}(\eta)$ of the generic point $\eta \in \mathbb{P}^{1}=\mathscr{L}$. Since $\mathbb{k}(\eta)$ is a $c_{1}$-field, by Tsen's theorem, the curve $L_{\eta}$ is isomorphic to $\mathbb{P}_{\mathbb{k}(\eta)}^{1}$. Consider the generic surface $F_{\eta}:=\pi^{-1}\left(L_{\eta}\right)$ over $\mathbb{k}(\eta)$. Then the morphism, $\pi_{\eta}: F_{\eta} \rightarrow L_{\eta}$ 
defines a standard conic bundle structure on the surface $F_{\eta}$ over the non-closed field $\mathbb{k}(\eta)$. The discriminant divisor of the fibration $\pi_{\eta}$ is $\Delta_{\eta}:=\Delta \cdot L_{\eta}$ and $\operatorname{deg} \Delta_{\eta}=\mathscr{L} \cdot \Delta \leq 3$. Corollary 5.4 implies that $F_{\eta}$ is rational over $\mathbb{k}(\eta)$. This implies the rationality of $X$ over $\mathbb{k}$.

From Proposition 8.2 we immediately obtain the following.

5.6.1. Corollary. Let $\pi: X \rightarrow \mathbb{P}^{2}$ be a conic bundle with discriminant curve $\Delta$ of degree $\operatorname{deg} \Delta \leq 4$. Then $X$ is rational.

\section{The Artin-Mumford invariant}

A natural birational invariant of an algebraic variety is the cohomological Brauer-Grothendieck group

$$
\operatorname{Br}(X):=H_{\text {ét }}^{2}\left(X, \mathbb{G}_{\mathrm{m}}\right),
$$

where $\mathbb{G}_{\mathrm{m}}$ is the sheaf of invertible regular functions in the étale topology on $X$. This is a periodic Abelian group which can be expressed in the classical situation of smooth projective varieties over $\mathbb{C}$ in terms of complex-analytic invariants of a variety and the torsion in $H^{3}(X, \mathbb{Z})$. In particular, if $\operatorname{dim} X=3$ and $\mathrm{p}_{\mathrm{g}}(X)=0$, then

$$
\operatorname{Br}(X) \simeq H^{3}(X, \mathbb{Z})_{\text {tors }}
$$

The birational invariance of the last group can be established immediately using the theorem on resolution of singularities by means of blowups with smooth centers:

6.1. Proposition (AM72]). The torsion subgroup $H^{3}(X, \mathbb{Z})_{\text {tors }}$ is a birational invariant of a complete non-singular complex variety $X$ of any dimension. In particular, $H^{3}(X, \mathbb{Z})_{\text {tors }}=0$ if $X$ is rational.

There is a version of this statement valid in characteristic $p>0$. In this case one has to replace $H^{3}(X, \mathbb{Z})$ with étale $l$-adic cohomology group $H^{3}\left(X, \mathbb{Z}_{l}\right)$, where $l$ is different from $p$ AM72.

6.2. Remark. It is easy to see that $H^{3}(X, \mathbb{Z})_{\text {tors }}$ is also a stably birational invariant: if $X_{1} \times \mathbb{P}^{n_{1}}$ is birationally equivalent to $X_{2} \times \mathbb{P}^{n_{2}}$, where $X_{1}$ and $X_{2}$ are smooth projective varieties, then

$$
H^{3}\left(X_{1}, \mathbb{Z}\right)_{\text {tors }} \simeq H^{3}\left(X_{2}, \mathbb{Z}\right)_{\text {tors }}
$$

Indeed, by Künneth formula one has

$$
H^{3}\left(X_{i} \times \mathbb{P}^{n_{i}}, \mathbb{Z}\right)_{\text {tors }} \simeq H^{3}\left(X_{i}, \mathbb{Z}\right)_{\text {tors }}
$$

The birational invariance of $H^{3}(X, \mathbb{Z})_{\text {tors }}$ was used in AM72 to construct counterexamples to the Lüroth problem in any dimension $\geq$ 
3. They first construct examples of unirational but not rational threedimensional varieties over $\mathbb{C}$ among conic bundles over rational surfaces with $H^{3}(X, \mathbb{Z})_{\text {tors }} \neq 0$.

It turns out that the group $H^{3}(X, \mathbb{Z})_{\text {tors }}$ is effectively computable in the case of conic bundles:

6.3. Theorem ([AM72], [Zag77, Th. 2]). Let $\pi: X \rightarrow S$ be a standard conic bundle over a rational surface $S$ with discriminant curve $\Delta$. Then

$$
H^{3}(X, \mathbb{Z})_{\text {tors }} \simeq \operatorname{Br}(X) \simeq(\mathbb{Z} / 2 \mathbb{Z})^{c-1},
$$

where $c$ is the number of connected components of $\Delta$.

This assertion states, in particular, that the number of connected components of the discriminant curve is a birational invariant:

6.3.2. Corollary. Let $\pi: X \rightarrow S$ and $\pi: X^{\prime} \rightarrow S^{\prime}$ be standard conic bundles over rational surfaces and let $\Delta$ and $\Delta^{\prime}$ be the corresponding discriminant curves. If $X$ and $X^{\prime}$ are birationally equivalent, then $\Delta$ and $\Delta^{\prime}$ have the same number of connected components.

The above result shows that to construct examples of non-rational three-dimensional varieties, it is sufficient to specify local invariants (3.10.1) and a disconnected curve $\Delta$ on some rational surface $S$.

6.3.3. Corollary. Let $\pi: X \rightarrow S$ be a standard conic bundle over a rational surface $S$ with discriminant curve $\Delta$. If $\Delta$ is disconnected, then $X$ is not rational. Moreover, $X$ is not stably rational.

The collection of local invariants (3.10.1) can be chosen in such a way that the resulting conic bundle $X / S$ is unirational. This construction together with the results of Sar82, Theorem 5.10] can also be used to produce examples of unirational non-rational conic bundles with trivial intermediate Jacobian and trivial $H^{3}(X, \mathbb{Z})_{\text {tors }}$ (see Theorem 9.6).

6.4. Example. Let $C_{1}, C_{2} \subset \mathbb{P}^{2}$ be smooth non-rational curves meeting each other transversely. Let $\sigma: S \rightarrow \mathbb{P}^{2}$ the blowup of the intersection points and let $\Delta_{i} \subset S$ be the proper transform of $C_{i}$. Take étale double covers $\tilde{\Delta}_{i} \rightarrow \Delta_{i}$. According to Proposition 3.10 there exists a standard conic bundle $\pi: X \rightarrow S$ with discriminant curve $\Delta=\Delta_{1} \cup \Delta_{2}$. By (6.3.1) the variety $X$ is not stably rational.

For smooth three-dimensional Fano varieties, the Brauer group is trivial. This follows from the classification (see [IP99, Tables $\S \S 12.2-$ 12.7]). However, for Fano varieties with singularities (more precisely, for their desingularizations) it can be non-trivial, as, for example, in 


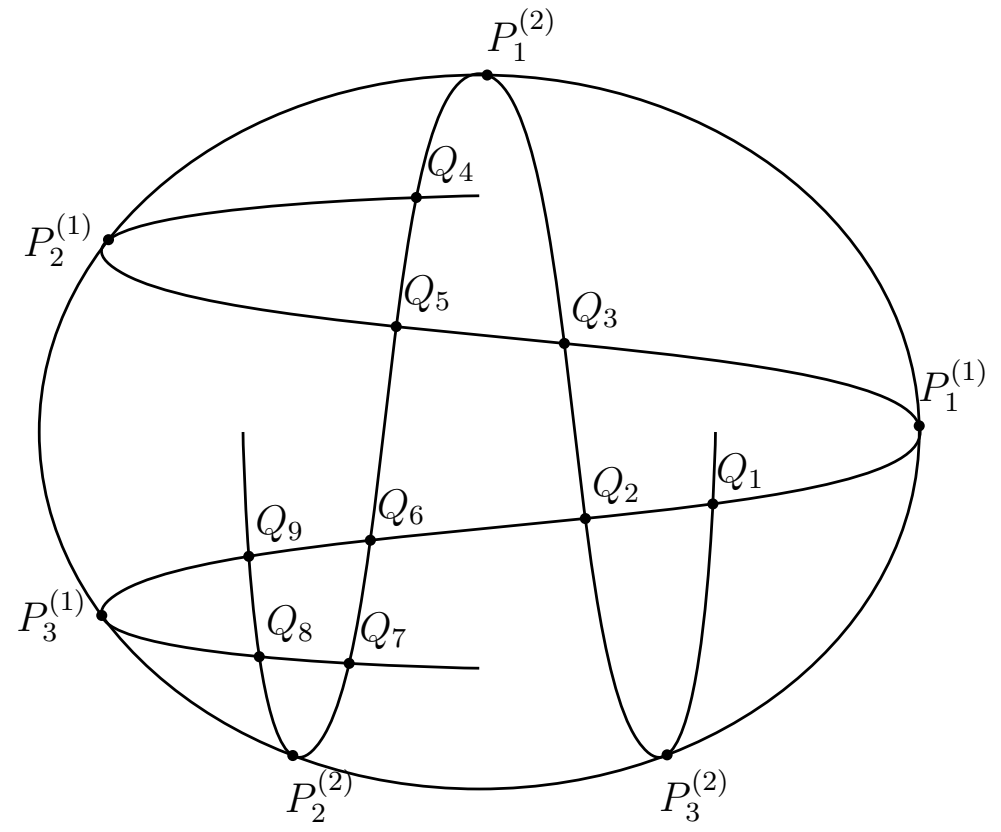

FIGURE 2.

AM72 where the simplest examples of non-rational unirational threedimensional varieties are given by double covers of $\mathbb{P}^{3}$ ramified in singular quartics, that is, by three-dimensional Fano varieties with double singularities. This example is constructed as follows.

6.5. Example. Let $C=\left\{f\left(x_{1}, x_{2}, x_{3}\right)=0\right\} \subset \mathbb{P}^{2}$ be a non-degenerate conic and let $E_{i}=\left\{g_{i}\left(x_{1}, x_{2}, x_{3}\right)=0\right\} \subset \mathbb{P}^{2}, i=1,2$ be smooth cubic curves such that

$$
\begin{aligned}
E_{i} \cap C & =\left\{P_{1}^{(i)}, P_{2}^{(i)}, P_{3}^{(i)}\right\}, \quad i=1,2, \\
E_{1} \cap E_{2} & =\left\{Q_{1}, \ldots, Q_{9}\right\} .
\end{aligned}
$$

where $P_{1}^{(1)}, \ldots P_{3}^{(2)}, Q_{1}, \ldots, Q_{9}$ are mutually distinct points, $E_{i}$ meet $C$ tangentially at $P_{1}^{(i)}, P_{2}^{(i)}, P_{3}^{(i)}$, and $E_{1}$ meets $E_{2}$ transversally at $Q_{1}, \ldots, Q_{9}$ (see Fig. 2). Since curves of degree 3 cut out a complete linear system on $C$, we have

$$
\sum_{i, j} P_{j}^{(i)}=\left.B\right|_{C}
$$

for some third cubic curve $B$, that is, $\left.\left(E_{1}+E_{2}\right)\right|_{C}=\left.2 B\right|_{C}$, as a cycle on $C$. Let $h\left(x_{1}, x_{2}, x_{3}\right)=0$ be an equation of $B$. For suitable choice of $h$ (up to scalar multiplication) we can write

$$
g_{1} g_{2}=h_{25}^{2}-4 f s
$$


for some $s=s\left(x_{1}, x_{2}, x_{3}\right)$ of degree 4 . Let $S \subset \mathbb{P}_{x_{0}, \ldots, x_{3}}^{3}$ be the quartic surface given by a homogeneous polynomial:

$$
f\left(x_{1}, x_{2}, x_{3}\right) x_{0}^{2}+h\left(x_{1}, x_{2}, x_{3}\right) x_{0}+s\left(x_{1}, x_{2}, x_{3}\right)=0 .
$$

It is easy to see that $S$ has exactly 10 nodes and no other singularities. Next, let $Y$ be the double cover of $\mathbb{P}^{3}$ (the "double solid") branched over $S$. Clearly, $Y$ can be given in the weighted projective space $\mathbb{P}(1,1,1,1,2)$ by the weighted homogeneous polynomial:

$$
x_{4}^{2}+f x_{0}^{2}+h x_{0}+s=0, \quad \operatorname{deg} x_{0}=\cdots=\operatorname{deg} x_{3}=1, \quad \operatorname{deg} x_{4}=2 .
$$

Let $\tilde{Y} \rightarrow Y$ be the blowup of the point $O:=(0: 0: 0: 1: 0)$ and let $\pi_{Y}$ : $Y \rightarrow \mathbb{P}^{2}$ be the morphism induced by the projection from $O$. Then $\pi_{Y}$ is a singular conic bundle whose discriminant locus $\Delta_{Y} \subset \mathbb{P}^{2}$ is $E_{1} \cup E_{2}$. A standard form of $\pi_{Y}$ is a conic bundle over the surface $S$ which is the blowup of $\mathbb{P}^{2}$ in $Q_{1}, \ldots, Q_{9}$ and the corresponding discriminant curve is a disjoint union of proper transforms of $E_{1}$ and $E_{2}$.

This example was generalized in IKP14 and [PS16d (see also [Bea16]) to some other types of singular Fano threefolds.

The birational invariance of the cohomological Brauer was used also to construct counterexamples to the E. Noether problem of the rationality of fields of invariants for finite linear groups operating on vector spaces over an algebraically closed field [Sal84], Bog88], [Sha91]. The problem can be formulated in geometrical terms as follows. Let $G$ be a finite group acting by linear transformations on a projective space $\mathbb{P}^{n}$. The problem is whether the quotient $X=\mathbb{P}^{n} / G$ is rational. See [CTS07], [Pro10], [Kan01] for surveys on this subject.

\section{Intermediate JaCOBIANS AND PRYM VARIETIES}

Let $X$ be a smooth projective three-dimensional variety over $\mathbb{C}$ such that $H^{1}\left(X, \mathscr{O}_{X}\right)=H^{3}\left(X, \mathscr{O}_{X}\right)=0$. For example, this holds for rationally connected varieties. Then we have the Hodge decomposition in the following form:

$$
H^{3}(X, \mathbb{C})=H^{2,1}(X) \oplus H^{1,2}(X) .
$$

Therefore the integration of $(2,1)$-forms over 3 -dimensional cycles determines an embedding $H_{3}(X, \mathbb{Z}) /($ torsion $) \hookrightarrow H^{2,1}(X)^{\vee}$ as a full rank lattice into the complex vector space $H^{2,1}(X)^{\vee}$. The alternating integral-valued intersection form on $H^{3}(X, \mathbb{Z})$ is unimodular by Poincaré duality, and the corresponding Hermitian form on $H^{2,1}(X)^{\vee}$ is positive definite. Therefore, by the Riemann-Frobenius criterion, the complex torus

$$
\mathrm{J}(X):=H^{2,1}(X)^{\vee} / \operatorname{image}\left(H^{3}(X, \mathbb{Z})\right)
$$


is a principally polarized Abelian variety with polarization divisor $\Theta$.

7.1. Definition. In the preceding notation, the pair $(\mathrm{J}(X), \Theta)$ is called the intermediate Jacobian of the variety $X$.

Applications of intermediate Jacobians in birational geometry are based on the following two observations.

7.2. Proposition. (i) Every principally polarized Abelian variety $(A, \Theta)$ can be decomposed in a unique way into a direct product of principally polarized simple Abelian varieties

$$
(A, \Theta)=\bigoplus_{i=1}^{m}\left(A, \Theta_{i}\right)
$$

(ii) (see [CG72] and also Tyu73, Lecture 1], [Tyu80, Proposition 1.2.8]) Let $X^{\prime} \rightarrow X$ be the blow-up with a smooth irreducible center $C \subset X$ (that is, $C$ is either a curve or a point). Then there is the following isomorphism of principally polarized Abelian varieties

$$
\mathrm{J}\left(X^{\prime}\right) \simeq \begin{cases}\mathrm{J}(X), & \text { if } C \text { is a point } \\ \mathrm{J}(X) \oplus \mathrm{J}(C), & \text { if } C \text { is a curve, }\end{cases}
$$

where $\mathrm{J}(C)$ is the Jacobian of the curve $C$ with the principal polarization determined by the Poincaré divisor $\Theta$.

This proposition and Hironaka's theorem on resolution of indeterminacies of rational maps immediately imply the birational invariance of that part (the so-called Griffiths component) of the decomposition (7.2.1) which is the product of those factors which are not Jacobians of curves. In other words, if one denotes by $\mathrm{J}_{\mathrm{G}}(X)$ the product of the components in (7.2.1) which are not Jacobians of curves, then $\mathrm{J}_{\mathrm{G}}(X)$ is a birational invariant. In particular, we have the following

7.3. Corollary. If the variety $X$ is rational, then $\mathrm{J}_{\mathrm{G}}(X)=0$.

Thus, to prove the non-rationality of some three-dimensional varieties one should be able to distinguish principally polarized Abelian varieties from Jacobians of curves.

It turns out that intermediate Jacobians of three-dimensional varieties having a structure of standard conic bundle can be described as so called Prym varieties. These varieties were studied first in papers of F. Prym, W. Wirtinger, F. Schottky, and H. Jung in connection with the Schottky problem of distinguishing the Jacobians of curves in the moduli spaces of principally polarized Abelian varieties (see [Bea77a], Cle75]). 
D. Mumford was the first who drew attention to the possibility of applications of Prym varieties to the birational geometry of threedimensional varieties in the appendix to the paper of [CG72]. He also studied double covers $\tilde{\Delta} \rightarrow \Delta$ of smooth curves $\Delta$ from the point of view of distinguishing their $\operatorname{Prym}$ varieties $\operatorname{Pr}(\tilde{\Delta}, \Delta)$ from Jacobians of curves [Mum74]. Mumford's results were extended to singular curves with normal crossings in the subsequent papers [Bea77b], Bea77a], [Sho82, Sho84].

7.4. Definition. Let $(\tilde{\Delta}, \tau)$ be the pair consisting of a complete reduced (possibly reducible) curve $\tilde{\Delta}$ with at most ordinary double points and an involution $\tau$ on $\tilde{\Delta}$ (that is, $\tau^{2}=$ id) acting non-trivially on every irreducible component of the curve $\tilde{\Delta}$. Denote by $\Delta$ the quotient $\tilde{\Delta} / \tau$, and by $\tilde{\pi}: \tilde{\Delta} \rightarrow \Delta$ the corresponding double cover. The involution $\tau$ induces an involution $\tau^{*}$ on the Jacobian $\mathrm{J}(\tilde{\Delta})$, and $\pi$ induces the norm map $\mathrm{Nm}: \mathrm{J}(\tilde{\Delta}) \rightarrow \mathrm{J}(\Delta)$, where $\tilde{\pi}^{*} \circ \mathrm{Nm}:=\mathrm{id}+\tau^{*}$ and $\mathrm{Nm} \circ \tilde{\pi}^{*}=2$. The connected commutative algebraic group

$$
\operatorname{Pr}(\tilde{\Delta}, \Delta):=\operatorname{ker}(\mathrm{Nm})^{0}=\left(\mathrm{id}-\tau^{*}\right) \mathrm{J}(\tilde{\Delta})
$$

is called the (generalized) Prym variety of the pair $(\tilde{\Delta}, \tau)$, where the symbol " 0" denotes the identity component. It is easy to see that

$$
\operatorname{dim} \operatorname{Pr}(\tilde{\Delta}, \Delta)=\mathrm{p}_{\mathrm{a}}(\Delta)-1 .
$$

7.4.1. The notion of polarization can be easily extended to generalized Prym varieties (being algebraic groups, they are not necessarily Abelian varieties when $\Delta$ is singular). Under certain conditions, $\operatorname{Pr}(\tilde{\Delta}, \Delta)$ is an Abelian variety the polarization of which is divisible by 2 and after the division becomes principal. This is true, for example, if the Beauville conditions (3.8.1) are satisfied (see [Bea77a] or [Sho84, Theorem 3.5]). For applications one has to impose a stronger condition:

for every decomposition $\Delta=\Delta_{1}+\Delta_{2}$ with $\Delta_{i}>0$ we have $\#\left(\Delta_{1} \cap \Delta_{2}\right) \geq 4$.

The condition (7.4.2) implies that $\Delta$ is a stable curve. In particular, if a curve $\Delta$ satisfies (7.4.2), then the canonical linear system $\left|\omega_{\Delta}\right|$ is base point free and defines the canonical morphism $\Delta \rightarrow \mathbb{P}^{\mathrm{p}_{\mathrm{a}}(\Delta)-1}$ which is finite onto its image (see [CFHR99]).

The following theorem was proved by Shokurov [Sho84]. For weaker versions see [Mum74, [Bea77a], [Deb89].

7.5. Theorem. Let $(\tilde{\Delta}, \tau)$ be a pair consisting of a curve $\tilde{\Delta}$ of arithmetic genus $2 g-1$, and an involution $\tau$ on $\tilde{\Delta}$ satisfying the Beauville 
conditions (3.8.1), as well as, the condition (17.4.2). Let $\Delta=\tilde{\Delta} / \tau$ be the quotient curve, and let $\operatorname{Pr}(\tilde{\Delta}, \Delta)$ be the corresponding Prym variety with the polarization divisor $\Xi$ so that $\mathrm{p}_{\mathrm{a}}(\Delta)=g$ and $\operatorname{dim} \operatorname{Pr}(\tilde{\Delta}, \Delta)=$ $g-1$. Then $(\operatorname{Pr}(\tilde{\Delta}, \Delta), \Xi)$ is the Jacobian of a curve or a product of Jacobians of curves only in the following cases:

(i) $\Delta$ is a hyperelliptic curve;

(ii) $\Delta$ is a trigonal curve (this case is considered in Rec74);

(iii) $\Delta$ is a quasi-trigonal curve;

(iv) $\Delta$ is a plane quintic curve and the corresponding double cover is given by an even theta-characteristic.

Here, as usual, a (not necessarily smooth) curve $\Delta$ is said to be hyperelliptic (resp. trigonal) if there exists a finite morphism $\Delta \rightarrow \mathbb{P}^{1}$ of degree 2 (resp. 3). A curve $\Delta$ is said to be quasi-trigonal if obtained from a hyperelliptic curve by identifying two smooth points.

7.5.1. Remark. The canonical map $\phi_{\Delta}: \Delta \rightarrow \mathbb{P}^{g-1}$ of the curves listed in Theorem [7.5 satisfy the following properties.

(i) If the curve $\Delta$ is hyperelliptic, then the map $\phi_{\Delta}$ is a finite morphism of degree 2 .

(ii) If $\Delta$ is a trigonal non-hyperelliptic curve, then the map $\phi_{\Delta}$ is an embedding and its image has a one-dimensional family of 3-secant lines whose intersections with $\phi_{\Delta}(\Delta)$ generate the linear series $\mathfrak{g}_{3}^{1}$.

(iii) If $\Delta$ is a quasi-trigonal non-hyperelliptic curve, then the map $\phi_{\Delta}$ is an embedding and its image lies on a two-dimensional cone with vertex at a singular point. Here the generators of the cone are 3 -secant lines of the curve $\phi_{\Delta}(\Delta)$.

(iv) If $\Delta$ is a plane quintic, then $\phi_{\Delta}$ is an embedding and its image lies on the Veronese surface.

7.5.2. Remark. Assume that $\tilde{\Delta}_{1} \cap \tilde{\Delta}_{2}=\left\{P_{1}, P_{2}\right\}$ and let $\tilde{\Delta}_{i}^{\prime}, i=1,2$ be curves obtained from $\tilde{\Delta}_{i}$ by identifying the points $P_{1}$ and $P_{2}$ (see Fig (3).

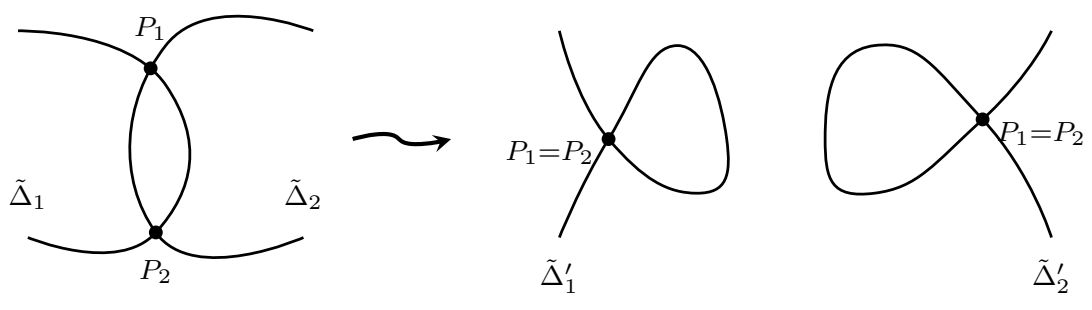

Figure 3. 
Then $\operatorname{Pr}(\tilde{\Delta}, \Delta)=\operatorname{Pr}\left(\tilde{\Delta}_{1}^{\prime}, \Delta_{1}^{\prime}\right) \times \operatorname{Pr}\left(\tilde{\Delta}_{2}^{\prime}, \Delta_{2}^{\prime}\right)$, where $\operatorname{Pr}\left(\tilde{\Delta}_{i}^{\prime}, \Delta_{i}^{\prime}\right)$ are Prym varieties for $\tilde{\Delta}_{i}^{\prime}$ with the induced involution (see Sho84, Corollary 3.16]). This is the reason for the condition (17.4.2) to be included in the hypothesis of the theorem.

Prym varieties are used in the three-dimensional birational geometry mainly in the following situation. Let $\pi: X \rightarrow S$ be a standard conic bundle over a smooth projective rational surface. Let $\Delta \subset S$ be the discriminant curve of $\pi$, and let $\tilde{\Delta}$ be the curve parameterizing irreducible components of degenerate conics over $\Delta$. Then if $\Delta \neq \varnothing$, then $\Delta$ is a reduced curve with normal crossings, and $\pi$ induces a double cover $\tilde{\pi}: \tilde{\Delta} \rightarrow \Delta$ satisfying Beauville conditions (3.8.1).

For the following theorem, see [Bea77b, Ch. 3], [Bel85], [Bea89].

7.6. Theorem. Let $\pi: X \rightarrow S$ be a standard conic bundle over a rational surface $S$ with discriminant curve $\Delta \subset S$ and let $\tilde{\Delta} \rightarrow \Delta$ be the corresponding double cover. Then the intermediate Jacobian $\mathrm{J}(X)$ is isomorphic as a principally polarized abelian variety to the Prym variety $\operatorname{Pr}(\tilde{\Delta}, \Delta)$.

A. Beauville considered the case $S=\mathbb{P}^{2}$ and obtained as a consequence of Theorems 7.5 and 7.6 the following statement [Bea77b, Théorème 4.9]: if $S=\mathbb{P}^{2}$, and $\operatorname{deg} \Delta \geq 6$, then the variety $X$ is nonrational since in this case $\operatorname{Pr}(\tilde{\Delta}, \Delta)$ is neither the Jacobian of a curve nor a product of Jacobians of curves.

Intermediate Jacobian was used to prove non-rationality of many types of (even singular) Fano threefolds, see e.g. [Bea77b, Ch. 5], [Cle83], CM77a, CM77b], [CPS15], [PV83], [Ehn85], AB92].

\section{BirationAl TRANSFORMATIONS}

The following result, based on the construction of [Tyu75], was obtained by I. Panin in Pan80. It is related to the exceptional case (ii) of Conjecture 1.3 .

8.1. Proposition ([Pan80]). Let $\pi: X \rightarrow \mathbb{P}^{2}$ be a standard conic bundle with discriminant curve $\Delta \subset \mathbb{P}^{2}$ of degree 5 . Suppose that the corresponding double cover $\tilde{\pi}: \tilde{\Delta} \rightarrow \Delta$ associated to $\pi$ is given by an even theta-characteristic. Then $X$ is rational. Moreover, $\pi: X \rightarrow \mathbb{P}^{2}$ is fiberwise birational to the conic bundle obtained by blowing up of $\mathbb{P}^{3}$ along a smooth curve of degree 7 and genus 5, see Example 3.4.3.

Sketch of the proof. According to Lemma 3.10, such a conic bundle exists and any two of them are birationally equivalent over $\mathbb{P}^{2}$. Thus it is sufficient to prove the rationality of a particular one. Since $\omega_{\Delta}=\mathscr{O}_{\Delta}(2)$, the curve $\Delta$ is neither hyperelliptic nor (quasi-)trigonal 
because its canonical model $\phi_{K_{\Delta}}(\Delta) \subset \mathbb{P}^{5}$ lies in the Veronese surface. As $\tilde{\pi}$ satisfies the conditions (3.8.1) the Prym variety $\operatorname{Pr}(\tilde{\Delta}, \Delta)$ is a principally polarized Abelian variety (see 7.4.1). Let $\Xi$ be the polarization divisor. By [Mum74, §7], [Sho84, Prop. 5.4] $\operatorname{dim} \operatorname{Sing}(\Xi) \leq 1$. Therefore, $\operatorname{Pr}(\tilde{\Delta}, \Delta)$ is not a product of two or more principally polarized Abelian varieties. Thus the $\operatorname{Prym}$ variety $\operatorname{Pr}(\tilde{\Delta}, \Delta)$ is (by [Mas76] and [Tyu75]) isomorphic as a principally polarized Abelian variety to the Jacobian $\mathrm{J}(\Gamma)$ of a smooth curve of genus 5 . One can show that the curve $\Gamma$ cannot be hyperelliptic nor trigonal (see Sho84, Mas76], [Tyu75]). Thus the canonical image $\Gamma \subset \mathbb{P}^{4}$ is a complete intersection of three quadrics $Q_{1} \cap Q_{2} \cap Q_{3}$. In other words, $\Gamma$ is the base locus of a net of quadrics $|2 H-\Gamma|$, where $H$ is a hyperplane in $\mathbb{P}^{4}$.

8.1.1. Definition ([Tyu75, Thyu76]). Let $\mathcal{C}$ be a net of quadrics in $\mathbb{P}^{2 m}$ such that the base locus $\operatorname{Bs}(\mathcal{C})$ is a smooth variety. Then a general quadric in the net $\mathcal{C}$ is smooth and degenerate quadrics in $\mathcal{C}$ are parametrized by a curve $\Delta \subset \mathcal{C} \simeq \mathbb{P}^{2}$. A general quadric $Q_{s}$ for $s \in \Delta$ has two families of $m$-dimensional linear subspaces. This defines a double cover $\tilde{\Delta} \rightarrow \Delta$ ramified exactly over singular points of $\Delta$. This double cover is called the invariant of the net $\mathcal{C}$.

8.1.2. Theorem ([Tyu75, Bea77b, Théorème 6.3]). Let $\mathcal{C}$ be a net of quadrics in $\mathbb{P}^{4}$ such that the base locus $\operatorname{Bs}(\mathcal{C})$ is a smooth curve $\Gamma$. Let $\tilde{\Delta} \rightarrow \Delta$ be the invariant of the net $\mathcal{C}$. Then the Prym variety $\operatorname{Pr}(\tilde{\Delta}, \Delta)$ is isomorphic to the Jacobian $\mathrm{J}(\Gamma)$ of $\Gamma$ as a principally polarized Abelian variety.

Now starting from this net of quadrics $|2 H-\Gamma|$ we build a standard conic bundle $\pi: X \rightarrow \mathbb{P}^{2}$ with the given double cover $\tilde{\Delta} \rightarrow \Delta$ and prove the rationality of $X$.

8.1.3. Lemma. Let $\Gamma \subset \mathbb{P}^{4}$ be a smooth canonical non-trigonal curve of genus 5 and let $\mathcal{C}$ be the net of quadrics passing through $\Gamma$. Let $\tilde{\Delta} \rightarrow \Delta$ be the invariant of the net $\mathcal{C}$. Fix a point $P \in \Gamma$. Let $\Gamma_{0} \subset \mathbb{P}^{3}$ be the projection of $\Gamma$ from $P$ and let $X \rightarrow \mathbb{P}^{3}$ be the blowup of $\Gamma_{0}$. Then the discriminant curve of the standard conic bundle $\pi: X \rightarrow \mathbb{P}^{2}$ constructed in Example 3.4 .3 is isomorphic to $\Delta$ and the corresponding double cover coincides with $\tilde{\Delta} \rightarrow \Delta$.

Sketch of the proof. Let $T_{P, \mathbb{P}^{4}}$ be the tangent space to $\mathbb{P}^{4}$ at $P$. We identify the target of the projection $\mathbb{P}^{4} \rightarrow \mathbb{P}^{3}$ from $P$ with the projectivization $\mathbb{P}\left(T_{P, \mathbb{P}^{4}}\right)$. Consider the following family of conics in $\mathbb{P}\left(T_{P, \mathbb{P}^{4}}\right)$. For each points $s \in \mathcal{C} \simeq \mathbb{P}^{2}$ we consider the conic $C_{s}$ which is the projectivized tangent cone to the quadric $Q_{s} \cap T_{P, \mathbb{P}^{4}}$ at the point $P$. Since $\Gamma$ is a smooth complete intersection of the members $Q_{s}, s \in \mathcal{C}$, 
none of the quadrics $Q_{s}$ has singular points on $\Gamma$, in particular at $P$. Therefore, we have one of the following possibilities:

$$
\begin{aligned}
& \operatorname{corank}\left(Q_{s}\right)=0 \Longleftrightarrow C_{s} \text { is smooth, } \\
& \operatorname{corank}\left(Q_{s}\right)=1 \Longleftrightarrow C_{s}=\mathbb{P}^{1} \vee \mathbb{P}^{1}, \\
& \operatorname{corank}\left(Q_{s}\right)=2 \Longleftrightarrow C_{s} \text { is a double line. }
\end{aligned}
$$

Moreover, each $C_{s}$ meets $\Gamma_{0}$ at $8-2=6$ points.

Conversely, if $C \subset T_{P, \mathbb{P} 4}$ is a (possibly degenerate) conic meeting $\Gamma_{0}$ at 6 points, then there is a quadric $Q \in \mathcal{C}$ such that $C$ is the projectivized tangent cone to $Q$ at $P$. As in Example 3.4.3, by blowing up $\Gamma_{0}$ we obtain a conic bundle $\pi: X \rightarrow \mathbb{P}^{2}$ whose fibers are proper transforms of conics $C_{s}$. In particular, the degeneracies of $\pi: X \rightarrow \mathbb{P}^{2}$ correspond precisely to the given cover $\tilde{\pi}: \tilde{\Delta} \rightarrow \Delta$ from which the net of quadrics was built.

By Theorems 8.1 .2 and 7.6 we have

$$
\operatorname{Pr}(\tilde{\Delta}, \Delta) \simeq \mathrm{J}(\Gamma) \simeq \mathrm{J}(X)
$$

Since $X$ is rational, Proposition 8.1 follows.

8.2. Proposition ([Sar81, Proposition 2.4]). Let $\pi: X \rightarrow S$ be a standard conic bundle. Let $\alpha: S_{1} \rightarrow S$ be the blowup with center at a point $s \in S$. Then there exists a standard conic bundle $\pi_{1}: X_{1} \rightarrow S_{1}$ and a birational map $\psi: X_{1} \rightarrow X$ such that the diagram

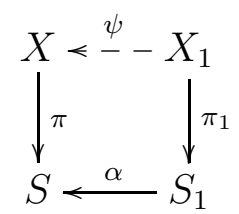

is commutative. More precisely, if $\pi^{-1}(s)$ is a smooth conic, then $\psi$ is regular and is the blowup of $\pi^{-1}(s)$. If $\pi^{-1}(s)$ is a degenerate conic, then (8.2.1) can be completed to the following commutative diagram (cf. 4.1)

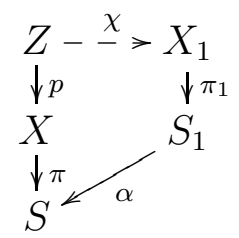

Here $p$ is the blowup of a reduced irreducible component of $\pi^{-1}(s)$ and $\chi$ is a flop. 
Sketch of the proof. Let $\Delta \subset S$ be the discriminant curve and let $l:=$ $\pi^{-1}(s)_{\text {red }}$ be the fiber over $s$. There are three possibilities:

$$
s \in S \backslash \Delta, \quad s \in \Delta \backslash \operatorname{Sing}(\Delta), \quad \text { and } s \in \operatorname{Sing}(\Delta) .
$$

8.2.3. Case $s \in S \backslash \Delta$. Then we can take $X_{1}=X \times_{S} S_{1}$. In other words, $\psi: X_{1} \rightarrow X$ is the blowup with center $l$. Since the curve $l$ is smooth, $X_{1}$ is a smooth variety. Moreover, $\psi$ is a morphism in this case.

8.2.4. Case $s \in \Delta \backslash \operatorname{Sing}(\Delta)$. Then $\pi^{-1}(s)=l=l^{\prime} \vee l^{\prime \prime}$. Let $p: Z \rightarrow X$ be the blowup of $l^{\prime}$ and let $\bar{l}$ be the proper transform of $l^{\prime \prime}$. For the normal bundle of $\bar{l}$ in $Z$ we have

$$
\mathscr{N}_{\bar{l} / Z}=\mathscr{O}_{\mathbb{P}^{1}}(-1) \oplus \mathscr{O}_{\mathbb{P}^{1}}(-1) .
$$

Therefore, there exists an Atiyah-Kulikov flop $Z \rightarrow X_{1}$ with center $\bar{l}$. The anti-canonical divisors of $Z$ and $X_{1}$ are relatively nef over $S$. According to general theory Mor82 there exists a Mori extremal contraction $\pi_{1}: X_{1} \rightarrow S_{1}$ over $S$ that can be completed to the diagram (8.2.2), where $\pi_{1}$ is a standard conic bundle and $\alpha$ is a blowup of $s$.

8.2.5. Case $s \in \operatorname{Sing}(\Delta)$. Then $\pi^{-1}(s)=2 l$ is a double line. Let $p: Z \rightarrow X$ be the blowup of $l$. As above the anti-canonical divisor $-K_{Z}$ is nef over $S$ and there exists a flop $Z \rightarrow X_{1}$ at the negative section of the exceptional divisor $p^{-1}(l) \simeq \mathbb{F}_{3}$. Thus we obtain the diagram (8.2.2).

The transformations described in 8.2.3 8.2.5 are called elementary transformations of conic bundles. They are simplest examples of Sarkisov links.

8.3. Lemma ([Isk96b]). Let $S$ and $S^{\prime}$ be surfaces with rational singularities and let $\alpha: S \rightarrow S^{\prime}$ be a birational contraction. Let $\Delta \subset S$ be a connected reduced curve and let $\Delta^{\prime}:=\alpha(\Delta)$. Then $\mathrm{p}_{\mathrm{a}}(\Delta) \leq \mathrm{p}_{\mathrm{a}}\left(\Delta^{\prime}\right)$.

8.4. Lemma ([Isk91, Lemma 1]). Let $\pi: X \rightarrow S$ and $\pi^{\prime}: X^{\prime} \rightarrow S^{\prime}$ be fiberwise birational equivalent standard conic bundles over smooth rational surfaces and let $\Delta \subset S$ and $\Delta^{\prime} \subset S^{\prime}$ be the corresponding discriminant curves. Then we have

$$
\begin{aligned}
\operatorname{dim}\left|m K_{S}+n \Delta\right| & =\operatorname{dim}\left|m K_{S^{\prime}}+n \Delta^{\prime}\right| \quad \forall m \geq n>0 \\
\mathrm{p}_{\mathrm{a}}(\Delta) & =\mathrm{p}_{\mathrm{a}}\left(\Delta^{\prime}\right) \\
\mathrm{b}_{3}(X) & =\mathrm{b}_{3}\left(X^{\prime}\right) .
\end{aligned}
$$

Moreover, $\Delta$ is smooth if and only if so $\Delta^{\prime}$ is.

Note that (8.4.1) and (8.4.2) fail in the category $\mathbb{Q}$-conic bundles, see Example 11.4. 
Proof. First we note that elementary birational transformations as in Proposition 8.2 do not change $\mathrm{p}_{\mathrm{a}}(\Delta)$ as well as $\left|m K_{S}+n \Delta\right|$. Indeed, let $\alpha: S^{\prime} \rightarrow S$ be the blowing up of $s \in S$ and let $\pi^{\prime}: X^{\prime} \rightarrow S^{\prime}$ be a standard conic bundle obtained from $\pi$ by one of the elementary transformations 8.2.3. 8.2.5, Then

$$
\Delta^{\prime}= \begin{cases}\alpha^{*} \Delta & \text { if } s \in S \backslash \Delta \\ \alpha^{*} \Delta-E & \text { if } s \in \Delta\end{cases}
$$

where $E=\alpha^{-1}(s)$ is an exceptional divisor. Since $K_{S^{\prime}}=\alpha^{*} K_{S}+E$, we have

$$
m K_{S^{\prime}}+n \Delta^{\prime}=\alpha^{*}\left(m K_{X}+n \Delta\right)+ \begin{cases}m E & \text { if } s \in S \backslash \Delta \\ (m-n) E & \text { if } s \in \Delta .\end{cases}
$$

This proves (8.4.1) in the case where $X / S \rightarrow X^{\prime} / S^{\prime}$ is an elementary transformation. (8.4.2) in this case follows from Lemma 8.3.

In general case, applying transformations as in Proposition 8.2 we may replace $\pi$ and $\pi^{\prime}$ with fiberwise birational conic bundles so that $S=S^{\prime}$. In this case $\Delta=\Delta^{\prime}$ because the discriminant curve depends only on the generic fiber (see Lemma 3.3.5). The last equality follows from (3.6.3).

8.5. Proposition ([Isk87, Remark 7], [Isk91, Lemma 4]). Let $\pi: X \rightarrow$ $S$ be a standard conic bundle with discriminant curve $\Delta$. Let $E \subset S$ be a (-1)-curve on $S$ such that one of the following three conditions is satisfied:

(i) $E \cdot \Delta=0, E \not \subset \Delta$;

(ii) $E \cdot \Delta=1, E \not \subset \Delta$;

(iii) $E \cdot \Delta=1, E \subset \Delta$, and $E \cdot(\Delta-E)=2$.

Let $\alpha: S \rightarrow S^{\prime}$ be the contraction of $E$, then there exists a standard conic bundle $\pi^{\prime}: X^{\prime} \rightarrow S^{\prime}$ with discriminant curve $\Delta^{\prime}=\alpha(\Delta)$ birationally equivalent to $\pi: X \rightarrow S$.

Proof. We show that $X^{\prime}$ can be obtained from $X$ by means of explicit birational transformations. Denote $F:=\pi^{-1}(E)$. Since $\rho\left(X / S^{\prime}\right)=2$, the relative Mori cone $\overline{\mathrm{NE}}\left(X / S^{\prime}\right)$ is generated by two extremal rays.

We need the following easy fact.

8.5.1. Lemma. Let $\pi: X \rightarrow S$ be a conic bundle and let $E \subset S$ be a complete smooth rational curve such that $F:=\pi^{-1}(E)$ is a smooth surface. Let $C \subset F$ be a curve such that $\pi_{C}: C \rightarrow E$ is an isomorphism. Then

$$
K_{X} \cdot C=-2-(E)_{S}^{2}-\underset{34}{(C)_{F}^{2}}, \quad \operatorname{deg} \mathscr{N}_{C / X}=(E)_{S}^{2}+(C)_{F}^{2} .
$$


Case (i), Then $F$ is a geometrically ruled surface over $E \simeq \mathbb{P}^{1}$. Let $\Sigma \subset F$ be the exceptional section and let $n:=-(\Sigma)_{F}^{2}$. From (8.5.2) one has $K_{X} \cdot \Sigma=n-1$ and the normal bundle $\mathscr{N}_{\Sigma / X}$ is isomorphic to $\mathscr{O}_{\mathbb{P}^{1}}(-n) \oplus \mathscr{O}_{\mathbb{P}^{1}}(-1)$. If $n=0$ on $F$, then $F \simeq \mathbb{P}^{1} \times \mathbb{P}^{1}$ and $\Sigma \cdot F=-1$ on $X$, since $E^{2}=-1$ on $S$. Therefore, $F \subset X$ can be contracted along the pencil $|\Sigma|$ in the category of smooth varieties and the corresponding contraction is extremal and, in particular, projective. We obtain a standard conic bundle $\pi^{\prime}: X^{\prime} \rightarrow S^{\prime}$. If $n \geq 1$, then blowing $\Sigma$ up and contracting the proper transform of $F$, we obtain a standard conic bundle $\pi_{1}: X_{1} \rightarrow S$ over $S$ with geometric ruled surface $F_{1}=\pi^{\prime-1}(E)$ and the exceptional section $\Sigma_{1} \subset F_{1}$ whose self-intersection index is equal to $-(n-1)$. The birational transformation $X \rightarrow X_{1}$ is a Sarkisov link of type II. After $n$ similar transformations, we arrive at the situation considered above. See [Isk91, Lemma 4] or [Pro05, Lemma 5.4] for details.

Case (ii), Then $F$ is a smooth ruled surface with one blowup point. Let $C \subset F$ be a section with minimal self-intersection number $C^{2}=$ $-n$ on $F$. It is clear that $n \geq 1$ and that $C$ intersects exactly one component of a unique degenerate fiber of the ruling $F \rightarrow E$. We denote this component by $\Gamma$. Again by (8.5.2) one has

$$
K_{X} \cdot C=n-1 \quad \text { and } \quad \mathscr{N}_{C / X} \simeq \mathscr{O}_{\mathbb{P}^{1}}(-n) \oplus \mathscr{O}_{\mathbb{P}^{1}}(-1) .
$$

Suppose first, that $n=1$. Since $K_{X} \cdot C=0$, the divisor $-K_{X}$ is nef over $S^{\prime}$. Then we can make a flop $X \rightarrow Z$ and then the proper transform of $F$ becomes contractible to a curve, i.e there exists a Mori extremal contraction $Z \rightarrow X^{\prime}$ over $S^{\prime}$, and then $\pi^{\prime}: X^{\prime} \rightarrow S^{\prime}$ is a standard conic bundle. The transformation $X \rightarrow X^{\prime}$ is a type III Sarkisov link inverse to the transformation described in 8.2.4,

If $n \geq 2$, then we consider the blowup $p: Z \rightarrow X$ of $C$ and complete it to a Sarkisov link of type II (we use the notation of 4.1). Here $\chi: Z \rightarrow Z_{1}$ is a flop in the proper transform of $\Gamma$ and $q: Z_{1} \rightarrow X_{1}$ is the contraction of the proper transform of $F$ to a curve over $S$. The exceptional divisor $R:=p^{-1}(C)$ over $C$ is isomorphic to $\mathbb{F}_{n-1}$ and its negative section is disjoint from the flipping curve. Thus we obtain a standard conic bundle $\pi_{1}: X_{1} \rightarrow S$. Moreover, the inverse image $F_{1}=\pi_{1}^{-1}(E)$ is obtained by blowing up of $\mathbb{F}_{n-1}$ at a point that does not lie on the negative section. In particular, $F_{1}$ has a section $C_{1}$ with $C_{1}^{2}=-(n-1)$. Therefore, after $n-1$ transformations of the type we have considered, we arrive at the situation $n=1$.

Case (iii), Then the singular locus of the surface $F$ is a curve $\Sigma \subset F$ such that the restriction $\pi_{\Sigma}: \Sigma \rightarrow E$ is an isomorphism. Let $\nu: \tilde{F} \rightarrow F$ be the normalization and let $\tilde{\Sigma}:=\nu^{-1}(\Sigma)$. From the explicit equations in 3.3 one can see that the pair $(X, F)$ is $\log$ 
canonical, the surface $F$ has generically normal crossings along $\Sigma$ and $\nu: \tilde{F} \rightarrow F$ induces a morphism $\nu_{\Sigma}: \tilde{\Sigma} \rightarrow \Sigma$ of degree 2 branched at two points $\pi^{-1}(\overline{\Delta \backslash E}) \cap \Sigma$. The surface $\tilde{F}$ is a minimal rational ruled surface isomorphic to $\mathbb{F}_{e}$ for some $e \geq 0$ with ruling $\tilde{F} \rightarrow \tilde{E}$, where $\tilde{E}$ is a curve that parametrizes the irreducible components of the fibers of $F \rightarrow E$ and $\tilde{E} \rightarrow E$ is the double cover branched at two points $E \cap \overline{\Delta \backslash E}$.

By the projection formula we have

$$
F \cdot \Sigma=\pi^{*} E \cdot \Sigma=E \cdot \pi_{*} \Sigma=E^{2}=-1 .
$$

Therefore,

$$
\left(K_{X}+F\right) \cdot \nu_{*} \tilde{\Sigma}=\left(K_{X}+F\right) \cdot 2 \Sigma=2 K_{X} \cdot \Sigma-2 .
$$

On the other hand, since $(X, F)$ is log canonical, by the adjunction formula [Sho93, 3.1]

$$
\left.\nu^{*}\left(K_{X}+F\right)\right|_{F}=K_{\tilde{F}}+\tilde{\Sigma} .
$$

Hence,

$$
\left(K_{X}+F\right) \cdot \nu_{*} \tilde{\Sigma}=\left(K_{\tilde{F}}+\tilde{\Sigma}\right) \cdot \tilde{\Sigma}=2 \mathrm{p}_{\mathrm{a}}(\tilde{\Sigma})-2=-2 .
$$

Combining the above two relations (8.5.3) and (8.5.4), we obtain

$$
K_{X} \cdot \Sigma=0 .
$$

Let $m:=(\tilde{\Sigma})_{\tilde{F}}^{2}$. Let $\Lambda$ be the ruling of $\mathbb{F}_{e}=\tilde{F} \rightarrow \tilde{E}$. Since $\pi: X \rightarrow S$ is a standard conic bundle, we have $\nu^{*} K_{X} \cdot \Lambda=-1$. Hence $\nu^{*} K_{X}=$ $-\tilde{\Sigma}+a \Lambda$ for some $a \in \mathbb{Z}$. The relation (8.5.5) implies $a=m$, i.e. $\nu^{*} K_{X}=-\tilde{\Sigma}+m \Lambda$. Therefore,

$$
\left(\nu^{*} K_{X}\right)^{2}=(-\tilde{\Sigma}+m \Lambda)^{2}=m-2 m=-m .
$$

On the other hand, using (3.11.1) we can compute:

$$
\begin{aligned}
-m=\left(\nu^{*} K_{X}\right)^{2}=\pi_{*} \nu_{*} \nu^{*}\left(K_{X}^{2}\right) & =\pi_{*}\left(\pi^{*} E \cdot K_{X}^{2}\right)= \\
& =E \cdot \pi_{*}\left(K_{X}^{2}\right)=E \cdot\left(-4 K_{S}-\Delta\right)=3 .
\end{aligned}
$$

Hence $\tilde{F} \simeq \mathbb{F}_{3}$ and $\tilde{\Sigma}$ is the negative section of $\tilde{F} \rightarrow \tilde{E}$. Since any curve on $\tilde{F}$ is linearly equivalent to a convex linear combination of $\tilde{\Sigma}$ and $\Lambda$, we conclude that $-K_{X}$ is nef over $S^{\prime}$ and $\Sigma$ is the only curve contracted by $\left|-K_{X}\right|$ over $S^{\prime}$. Let $X \rightarrow Z$ be the flop in $\Sigma$. Then $Z$ has a Mori extremal contraction $Z \rightarrow X^{\prime}$ over $S^{\prime}$ and the induced morphism $\pi^{\prime}: X^{\prime} \rightarrow S^{\prime}$ is a standard conic bundle. The transformation described above is a type III Sarkisov link inverse to the transformation described in 8.2.5.

The proof of Proposition 8.5 is complete. 
8.6. Proposition ([sk91]). Let $\pi: X \rightarrow S$ be a standard conic bundle over a rational surface $S$ and let $\Delta \subset S$ be the discriminant curve. Assume that $\left|2 K_{S}+\Delta\right|=\varnothing$. Then $2 K_{S}+\Delta$ is not nef and there exists a standard conic bundle $\pi^{\sharp}: X^{\sharp} \rightarrow S^{\sharp}$ with discriminant curve $\Delta^{\sharp}$ and the following commutative diagram

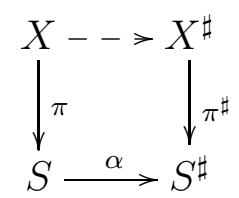

where $S^{\sharp} \simeq \mathbb{F}_{n}$ or $\mathbb{P}^{2}$, the morphism $\alpha$ is birational and $X \rightarrow X^{\sharp}$ is a birational map. Moreover, we have $\Delta^{\sharp} \cdot \Lambda^{\sharp} \leq 3$ for a fiber $\Lambda^{\sharp}$ of $\mathbb{F}_{n}$ (resp. $\left.\operatorname{deg} \Delta^{\sharp} \leq 5\right)$ if $S^{\sharp}=\mathbb{F}_{n}$ (resp. if $S^{\sharp}=\mathbb{P}^{2}$ ). In particular, the discriminant curve $\Delta$ is connected.

Proof. By the Riemann-Roch theorem

$$
\mathrm{h}^{0}\left(S, K_{S}+\Delta\right)+\mathrm{h}^{0}(S,-\Delta) \geq \mathrm{p}_{\mathrm{a}}(\Delta) \geq 1 .
$$

Therefore, $\left|K_{S}+\Delta\right| \neq \varnothing$. Apply Lemma 8.6 .2 below with $m=2$. Then $2 K_{S}+\Delta$ is not nef. If $C$ is a $(-1)$-curve on $S$ with $\left(2 K_{S}+\Delta\right) \cdot C<0$, then $\Delta \cdot C<2$. Then we can apply Proposition 8.5 and obtain a new conic bundle $\pi^{\prime}: X^{\prime} \rightarrow S^{\prime}$ with $\left|2 K_{S^{\prime}}+\Delta^{\prime}\right|=\varnothing($ see (8.4.1)). We can continue the process until we get a model with $S^{\sharp} \simeq \mathbb{F}_{n}$ or $\mathbb{P}^{2}$.

The connectedness of $\Delta^{\sharp}$ is easy to check on $S^{\sharp}=\mathbb{F}_{n}$ and $\mathbb{P}^{2}$. On the other hand, the number of connected components of the discriminant curve is preserved under our birational transformations.

8.6.2. Lemma (【sk91). Let $S$ be a smooth rational projective surface and $\Delta$ a reduced effective divisor on $S$ such that for any smooth rational curve $C \subset \Delta$ one has $C \cdot(\Delta-C) \geq 2$. Let $m$ and $n$ be positive integers such that $m>n$,

$$
\left|m K_{S}+n \Delta\right|=\varnothing, \quad\left|(m-1) K_{S}+n \Delta\right| \neq \varnothing .
$$

Then the divisor $m K_{S}+n \Delta$ is not nef and there exists an extremal ray $R \subset \overline{\mathrm{NE}}(S)$ such that $K_{S} \cdot R<0$ and $\left(m K_{S}+n \Delta\right) \cdot R<0$. Moreover, one of the following holds:

(i) there exists a base point free pencil of rational curves $\mathscr{L}$ on $S$ such that

$$
2 m-2 \leq n \Delta \cdot \mathscr{L}<2 m ;
$$

(ii) there exists a birational morphism $\varphi: S \rightarrow \mathbb{P}^{2}$ such that

$$
3 m-3 \leq n \operatorname{deg} \varphi(\Delta)<3 m .
$$


Proof. Put $\Delta_{k, l}:=k K_{S}+l \Delta$. We claim that the divisor $\Delta_{m, n}$ is not nef. Indeed, otherwise by the Riemann-Roch formula

$$
\mathrm{h}^{0}\left(S, \mathscr{O}_{S}\left(\Delta_{m, n}\right)\right)+\mathrm{h}^{0}\left(S, \mathscr{O}_{S}\left(-\Delta_{m-1, n}\right)\right) \geq \frac{1}{2} \Delta_{m, n} \cdot \Delta_{m-1, n}+1>0 .
$$

By our assumption,

$$
H^{0}\left(S, \mathscr{O}_{S}\left(\Delta_{m, n}\right)\right)=0
$$

Hence, $H^{0}\left(S, \mathscr{O}_{S}\left(-\Delta_{m-1, n}\right)\right) \neq 0$ and by our assumption $\Delta_{m-1, n}=0$. In this case, $\Delta_{m, n}=K_{S}$ is not nef because $S$ is a rational surface, a contradiction.

Thus, the divisor $\Delta_{m, n}$ is not nef. We regard $\Delta_{m, n}$ as a linear function on the Mori cone $\overline{\mathrm{NE}}(S)$. Put

$$
\overline{\mathrm{NE}}_{+}(S)=\left\{z \in \overline{\mathrm{NE}}(S) \mid z \cdot K_{S} \geq 0\right\} .
$$

We claim that $\Delta_{m, n}$ is non-negative on $\overline{\mathrm{NE}}_{+}(S)$. Indeed, assume that $C \cdot K_{S}:=d>0$ and $C \cdot \Delta_{m, n}<0$ for some irreducible curve $C$. Then

$$
n C \cdot \Delta=C \cdot \Delta_{m, n}-m C \cdot K_{S}<-d m .
$$

Hence $C$ is a component of $\Delta$ and $C^{2} \leq C \cdot \Delta<-d m / n$. By the genus formula

$$
2 \mathrm{p}_{\mathrm{a}}(C)-2=C^{2}+C \cdot K_{S}<d-d m / n<0 .
$$

Hence $\mathrm{p}_{\mathrm{a}}(C)=0$. Then by our assumptions

$$
-2-d=-2-C \cdot K_{S}=C^{2} \leq C \cdot \Delta-2<-d m / n-2 .
$$

The contradiction shows that $\Delta_{m, n}$ is non-negative on $\overline{\mathrm{NE}}_{+}(S)$.

Thus, $\Delta_{m, n}$ is negative on some $K_{S}$-negative extremal ray $R \subset$ $\overline{\mathrm{NE}}(S)$. According to [Mor82 there are only three possibilities:

1) $R=\mathbb{R}_{\geq 0}[E]$, where $E$ is the (-1) -curve,

2) $S \simeq \mathbb{F}_{e}$ and $R=\mathbb{R}_{\geq 0}[L]$, where $L$ is the ruling,

3) $S \simeq \mathbb{P}^{2}$ and $R=\mathbb{R}_{\geq 0}[L]$, where $L \subset \mathbb{P}^{2}$ is a line.

In case 1), let $\sigma: S \rightarrow S^{\prime}$ be the contraction of $E$. Put $\Delta^{\prime}:=\sigma_{*} \Delta$ and $\Delta_{k, l}^{\prime}:=k K_{S^{\prime}}+l \Delta^{\prime}=\sigma_{*} \Delta_{k, l}$. Clearly, the divisor $\Delta^{\prime}$ is reduced, $\Delta^{\prime} \geq 0$, and $\left|\Delta_{m-1, n}^{\prime}\right| \neq \varnothing$. Moreover, $C^{\prime} \cdot\left(\Delta^{\prime}-C^{\prime}\right) \geq 2$ for any irreducible rational component $C^{\prime} \subset \Delta^{\prime}$. Further,

$$
\Delta_{m, n}=\sigma^{*} \Delta_{m, n}^{\prime}-\left(\Delta_{m, n} \cdot E\right) E .
$$

Since $\Delta_{m, n} \cdot E<0$, one can see that the divisor $\Delta_{m, n}^{\prime}$ is not effective, as does the divisor $\Delta_{m, n}$. Therefore, all the conditions of our lemma are satisfied for $S^{\prime}$ and $\Delta^{\prime}$. Thus it is sufficient to show that either (i) or (ii) holds on $S^{\prime}$. Continuing the process of contraction of extremal $(-1)$-curves, we arrive at the situation of 2 ) or 3 ). 
In case 2) we have

$$
-2 \leq \Delta_{m-1, n} \cdot L+K_{S} \cdot L=\Delta_{m, n} \cdot L=n \Delta \cdot L-2 m<0,
$$

Put $\mathscr{L}:=|L|$. Then $2 m-2 \leq n \Delta \cdot \mathscr{L}<2 m$.

In case 3) we have

$$
-3 \leq \Delta_{m-1, n} \cdot L+K_{S} \cdot L=n \Delta \cdot L-3 m<0 .
$$

Hence, $3 n-3 \leq n \operatorname{deg} \varphi(\Delta)<3 m$. The lemma is proved.

Using similar arguments one can prove the following.

8.6.3. Lemma. Let $S$ be a smooth rational projective surface and $\Delta a$ reduced effective divisor on $S$ such that for any smooth rational curve $C \subset \Delta$ one has $C \cdot(\Delta-C) \geq 2$. Let $\Delta_{1}$ be a connected component of $\Delta$. Then

$$
\operatorname{dim}\left|K_{S}+\Delta\right| \geq \mathrm{p}_{\mathrm{a}}\left(\Delta_{1}\right)-1 \geq 0 .
$$

Furthermore, if $\left(K_{S}+\Delta\right) \cdot E<0$ for some irreducible curve $E$, then $E$ is a (-1)-curve disjoint from $\Delta$.

8.6.5. Corollary. Let $S$ be a smooth rational projective surface and let $\Delta$ be a reduced effective divisor on $S$ such that for any smooth rational curve $C \subset \Delta$ one has $C \cdot(\Delta-C) \geq 2$. Then there exists a sequence of contractions of $(-1)$-curves $f: S \rightarrow S^{\prime}$ contained in $S \backslash \operatorname{Supp}(\Delta)$ such that $K_{S^{\prime}}+f_{*} \Delta$ is nef.

8.7. Recall that a $\mathbb{R}$-divisor is said to be pseudo-effective if its class is a limit of classes of effective $\mathbb{R}$-divisors. Now let $S$ be a projective variety and let $\Delta$ be an $\mathbb{R}$-divisor on $S$. Define the effective threshold (see [Rei86], Rei91]) as follows

$$
\text { et }(S, \Delta):=\sup \left\{t \in \mathbb{R} \mid t K_{S}+\Delta \text { is pseudo-effective }\right\} \text {. }
$$

Assume now that $S$ is a smooth projective rational surface and $\Delta$ is an effective reduced divisor on $S$ such that $K_{S}+\Delta$ is nef. To compute $\lambda:=\operatorname{et}(S, \Delta)$ in this situation we can run $(K+\tau \Delta)$-MMP for suitable sequence $\tau=\tau_{i}$ and reduce the problem to the case where $\lambda K_{S}+\Delta$ is nef and then $S \simeq \mathbb{P}^{2}$ or $\mathbb{F}_{n}$. Then it is easy to see that $\lambda:=\operatorname{et}(S, \Delta) \in \frac{1}{2} \mathbb{Z} \cup \frac{1}{3} \mathbb{Z}$, see [Rei86] for details. In particular, et $(S, \Delta)$ is a rational number. This consideration shows also $\lambda K_{S}+\Delta$ has a Zariski decomposition

$$
\lambda K_{S}+\Delta \equiv N+P
$$

whose positive part $P$ is semi-ample and $P^{2}=0$. The negative part $N$ is contracted by $|n P|, n \gg 0$. Moreover, if et $(S, \Delta)=m / n$, then $\left|m K_{S}+n \Delta\right| \neq \varnothing$. Hence, the condition $\left|2 K_{S}+\Delta\right|=\varnothing$ in Conjecture 1.2 is equivalent to et $(S, \Delta)<2$.

From Proposition [5.6 we obtain the following. 
8.7.1. Corollary. Let $\pi: X \rightarrow S$ be a standard conic bundle over a rational surface $S$ and let $\Delta \subset S$ be the discriminant curve. Suppose that $\left|2 K_{S}+\Delta\right|=\varnothing$ and $X$ is not rational. Then there exists a diagram of the form (8.6.1) with $S^{\sharp} \simeq \mathbb{P}^{2}$ and $\operatorname{deg} \Delta^{\sharp}=5$. Moreover, the corresponding double cover $\tilde{\Delta}^{\sharp} \rightarrow \Delta^{\sharp}$ is defined by an odd theta characteristic (see Proposition 8.11).

8.7.2. Corollary ([Isk91]). Conjectures 1.2 and 1.3 are equivalent.

Proof. Assume that (i) or (ii) of Conjecture 1.3 holds. Then in the notation of Conjecture [1.3 we have $\left|2 K_{S^{\prime}}+\Delta^{\prime}\right|=\varnothing$ on $S^{\prime}$ and $\mid 2 K_{S}+$ $\Delta \mid=\varnothing$ on $S$ by Lemma 8.4. On the other hand, $X$ is rational (in the case (i) this follows from Proposition [5.6). Hence, $\mathrm{J}_{\mathrm{G}}(X)=0$.

Conversely, assume that $\left|2 K_{S}+\Delta\right|=\varnothing$. Apply Proposition 8.6. If $S^{\sharp} \simeq \mathbb{F}_{n}$, then the ruling of $\mathbb{F}_{n}$ give us the desired pencil on $S^{\sharp}$ and $S$ (because $\Delta^{\sharp}=\alpha(\Delta)$ ). Hence we have the case 1.3)(i) in this situation. Assume that $S^{\sharp} \simeq \mathbb{P}^{2}$ and $\operatorname{deg} \Delta^{\sharp} \leq 5$. If $\operatorname{deg} \Delta^{\sharp} \leq 4$, then the pencil of lines on $S^{\sharp} \simeq \mathbb{P}^{2}$ passing through a smooth point $P^{\sharp} \in \Delta^{\sharp}$ again gives us a pencil as in the case 1.3)(i) (after blowing up $P^{\sharp}$, see Proposition 8.2). Let deg $\Delta^{\sharp}=5$. Then $\mathrm{p}_{\mathrm{a}}\left(\Delta^{\sharp}\right)=\mathrm{p}_{\mathrm{a}}(\Delta)=6$. Since in this case $\mathrm{J}_{\mathrm{G}}(X)=$ 0 (see 1.2[ $(\star)]$, by Theorems 7.6 and 7.5 the corresponding double cover $\tilde{\Delta}^{\sharp} \rightarrow \Delta^{\sharp}$ is given by an even theta-characteristic. Applying Panin's construction 8.1 we obtain the case 1.3)(ii).

The following is a very weak version of Conjecture 1.3,

8.8. Proposition ([Isk91]). Let $\pi: X \rightarrow S$ be a standard conic bundle over a rational surface $S$ with discriminant curve $\Delta \subset S$. Assume that there exists a birational map $\Phi: X \rightarrow X^{\sharp}$ to another Mori fiber space $\pi^{\sharp}: X^{\sharp} \rightarrow S^{\sharp}$ such that $\Phi$ is not fiberwise. Then one of the following conditions is satisfied:

(i) there is a base point free pencil of curves of genus zero $\mathscr{L}$ on $S$ such that $\Delta \cdot \mathscr{L} \leq 7$;

(ii) there is a birational contraction $\varphi: S \rightarrow \mathbb{P}^{2}$ such that $\varphi(\Delta)=$ $\Delta^{\prime} \subset \mathbb{P}^{2}$ is a curve, not necessarily with normal crossings, of degree $\operatorname{deg} \Delta^{\prime} \leq 11$.

Proof. It follows from Theorem 12.2 below that $\left|4 K_{S}+\Delta\right|=\varnothing$. If $\left|2 K_{S}+\Delta\right|=\varnothing$, then the assertion follows from Proposition 8.6. Thus we can take $m \in\{3,4\}$ so that $\left|m K_{S}+\Delta\right|=\varnothing$, and $\left|(m-1) K_{S}+\Delta\right| \neq$ $\varnothing$. Then by Lemma 8.6.2 we obtain (i) or (ii),

8.9. Proposition ([Tre90, [Bea00, Proposition 4.2 b]). Let $\pi: X \rightarrow$ $\mathbb{P}^{2}$ be a standard conic bundle with smooth discriminant curve $\Delta$ of degree 5 such that the cover $\tilde{\Delta} \rightarrow \Delta$ is defined by an odd thetacharacteristic. Then $\pi: X \rightarrow \mathbb{P}^{2}$ is fiberwise birational to a standard 
conic bundle $\pi^{\prime}: X^{\prime} \rightarrow \mathbb{P}^{2}$ obtained from the projection of a smooth cubic hypersurface $Y_{3} \subset \mathbb{P}^{4}$ from a line (Example 3.4.2).

Note that the cubic $Y_{3} \subset \mathbb{P}^{4}$ can be naturally reconstructed from $\tilde{\Delta} \rightarrow \Delta$ : there is an isomorphism of principally polarized Abelian varieties $\operatorname{Pr}(\tilde{\Delta}, \Delta) \simeq \mathrm{J}(X)$ and the theta-divisor $\Theta$ of $\mathrm{J}(X)$ has a unique singular point whose tangent cone is isomorphic to the affine cone over $Y_{3} \subset \mathbb{P}^{4}$ Tyu71, Tyu80, Proposition 2.1.5], [Bea82].

\section{Conic Bundles over minimal surfaces}

In the case when the base of a conic bundle is a minimal rational surface the criterion 1.2 was proved by Shokurov [Sho84]. Below we reproduce his arguments.

9.1. Theorem. Let $\pi: X \rightarrow \mathbb{P}^{2}$ be a standard conic bundle and let $\Delta \subset \mathbb{P}^{2}$ be the discriminant curve. Then the following conditions are equivalent:

(i) $X$ is rational.

(ii) Either $\operatorname{deg} \Delta \leq 4$ or $\operatorname{deg} \Delta=5$ and the corresponding double cover $\tilde{\Delta} \rightarrow \Delta$ is defined by an even theta-characteristic.

(iii) The intermediate Jacobian $\mathrm{J}(X)$ is isomorphic as a principally polarized Abelian variety to a product of Jacobians of smooth curves, i.e. its Griffiths component $\mathrm{J}_{\mathrm{G}}(X)$ is trivial.

For the case $\operatorname{deg} \Delta \geq 6$ this theorem was proved by Beauville [Bea77b, Théorème 4.9]. In the case $\operatorname{deg} \Delta \leq 4$ the variety $X$ is rational by Corollary [5.6.1. The case $\operatorname{deg} \Delta=5$ follows from [Tyu73, §3], [Tyu75, [Mas76], [Pan80].

9.2. Theorem ([Sho84]). Let $\pi: X \rightarrow S$ be a standard conic bundle over a minimal rational ruled surface $S$, that $i s, S \simeq \mathbb{F}_{n}$ with $n \neq 1$, and let $\Delta \subset S$ be the discriminant curve. Denote by $\Lambda$ a fiber of the ruling $\mathbb{F}_{n} \rightarrow \mathbb{P}^{1}$. Then the following conditions are equivalent:

(i) $X$ is rational.

(ii) $\left|2 K_{S}+\Delta\right|=\varnothing$.

(iii) $\Delta \cdot \Lambda \leq 3$ (for suitable choice of the ruling $\mathbb{F}_{0} \rightarrow \mathbb{P}^{1}$ if $n=0$ ).

(iv) $\mathrm{J}_{\mathrm{G}}(X)=0$.

9.2.1. Remark. In the case $S=\mathbb{F}_{1}$, one can show that the equivalences (i) $\Leftrightarrow$ (ii) $\Leftrightarrow$ (iii) $\Leftrightarrow$ (iv) hold whenever $\Delta \cdot \Sigma>1$. If $\Delta \cdot \Sigma \leq 1$, then we can apply Proposition 8.5 to reduce the problem to $\mathbb{P}^{2}$. Thus the equivalence (i) $\Longleftrightarrow$ (iv) holds for $S=\mathbb{P}^{2}$ and $\mathbb{F}_{n}$ with arbitrary $n$.

9.3. Corollary. Conjectures 1.2 and 1.3 are true for $S=\mathbb{P}^{2}$ and $\mathbb{F}_{n}$, $n \geq 0$. 
Proof of Theorem 9.2. The implication (i) $\Longrightarrow$ (iv) is an immediate consequence of Corollary 7.3 and (iii) $\Longrightarrow$ (i) is a consequence of Propositions 5.6. The implication (iii) $\Longrightarrow$ (ii) is obvious.

Let us prove (ii) $\Longrightarrow$ (iii), Denote by $\Sigma$ the $(-n)$-section of $S=\mathbb{F}_{n}$. Write $\Delta \sim a \Sigma+b \Lambda$. We assume that $\left|2 K_{S}+\Delta\right|=\varnothing$ and $a \geq 4$. If $\Sigma \not \subset \Delta$, then clearly $b \geq$ an (see e.g. [Har77, Ch. V, Th. 2.17]). If $\Sigma \subset \Delta$, then

$$
(\Delta-\Sigma) \cdot \Sigma=b-a n+n \geq 2
$$

by (3.8.2). Thus, in both cases,

$$
b \geq \min \{a n, n(a-1)+2\} .
$$

Further,

$$
2 K_{S}+\Delta \sim(a-4) \Sigma+(b-2 n-4) \Lambda,
$$

where $b-2 n-4<(a-4) n$ because $\left|2 K_{S}+\Delta\right|=\varnothing$. This gives us $n=0$ and $b<4$. Replacing the ruling of $\mathbb{F}_{0}=\mathbb{P}^{1} \times \mathbb{P}^{1}$ with another one we get (iii).

It remains to prove the implication (iv) $\Longrightarrow$ (iii). Assume to the contrary that $\Delta \cdot \Lambda \geq 4$.

9.4. Lemma. In the above assumptions one of the following holds:

(i) $\left|K_{S}+\Delta\right|$ is very ample.

(ii) $\left|K_{S}+\Delta\right|$ is base point free but not very ample, the corresponding morphism is birational and contracts $\Sigma$. Moreover, $(\Delta-\Sigma) \cdot \Sigma=2$.

Proof. As above, we write

$$
\Delta \sim a \Sigma+b \Lambda, \quad K_{S}+\Delta \sim(a-2) \Sigma+(b-2-n) \Lambda .
$$

By our assumption $a \geq 4$. If $n=0$, then by symmetry we may assume that $b \geq 4$.

The linear system $\left|K_{S}+\Delta\right|$ is base point free if and only if

$$
b \geq a n+2-n .
$$

This relation is obvious if $n=0$. Thus we assume that $n \geq 2$. If $b \geq a$, then (9.4.1) holds. Consider the case $b<a n$. Then $\Sigma$ is a component of $\Delta$. Put $\Delta^{\prime}:=\Delta-\Sigma$. Then

$$
2 \leq \Delta^{\prime} \cdot \Sigma=b-a n+n
$$

which is equivalent to (9.4.1). Moreover, if $\left|K_{S}+\Delta\right|$ is not very ample, then in (9.4.1) the equality holds and we get (ii).

The following assertion can be proved similar to Lemma 9.4. 
9.5. Lemma. If the curve $\Delta$ does not satisfy the condition (7.4.2), that is, there exists a decomposition $\Delta=\Delta_{1}+\Delta_{2}$ with $\Delta_{1} \cdot \Delta_{2}=$ 2 , then up to permutation of $\Delta_{i}$ we may assume that $\Delta_{2}=\Sigma$ and $\left(K_{S}+\Delta\right) \cdot \Delta_{2}=0$. In particular, $n>0$. Thus $\Delta$ satisfies (7.4.2) if and only if the linear system $\left|K_{S}+\Delta\right|$ is very ample.

Now we are in position to finish the proof of the implication (iv) $\Longrightarrow$ (iii) in Theorem 9.2. Consider the morphism $\phi: S \rightarrow \mathbb{P}^{N}$ given by the linear system $\left|\omega_{S}(\Delta)\right|$ (see Lemma 9.4). From the exact sequence

$$
0 \longrightarrow \omega_{S} \longrightarrow \omega_{S}(\Delta) \longrightarrow \omega_{\Delta} \longrightarrow 0
$$

and the vanishing $H^{0}\left(S, \omega_{S}\right)=H^{1}\left(S, \omega_{S}\right)=0$ it follows that the linear system $\left|\omega_{S}(\Delta)\right|$ on $S$ restricts isomorphically to the canonical linear system $\left|\omega_{\Delta}\right|$ on $\Delta$. Therefore, $\phi$ induces the canonical morphism on $\Delta$

$$
\phi_{\Delta}: \Delta \rightarrow \mathbb{P}^{\mathrm{p}_{\mathrm{a}}(\Delta)-1} .
$$

According to Lemma 9.4 the morphism $\phi$ is birational. Hence $\Delta$ is not hyperelliptic.

Assume that $K_{S}+\Delta$ is very ample. Then $\phi_{\Delta}$ is an embedding and $\Delta$ satisfies the condition (7.4.2). By Theorem 7.5 the curve $\Delta$ is either trigonal, quasitrigonal or isomorphic to a plane quintic. The surface $\phi(S)$ is an intersection of quadrics. Therefore it contains all the 3secants of the curve $\phi(\Delta)$. If $\Delta$ is trigonal or quasitrigonal, then this implies that $\phi(S)$ is swept out by 3 -secants (see Remark 7.5.1), and these 3 -secants must be the images of the fibers $\Lambda$. Hence, in this case, $\Delta \cdot \Lambda \leq 3$ which contradicts our assumption. If $\Delta$ is a plane quintic, then the intersection of quadrics passing through $\phi(\Delta)$ is the Veronese surface. But this surface cannot be an image of $\mathbb{F}_{n}$ for $n \neq 1$. Again we get a contradiction.

Finally assume that the divisor $K_{S}+\Delta$ is not very ample. By Lemma 9.5 we have $\Delta=\Sigma+\Delta_{1}, \Sigma \cdot \Delta_{1}=2$, and $\left(K_{S}+\Delta\right) \cdot \Sigma=0$. Moreover the surface $\phi(S)$ is isomorphic to a cone over a rational normal curve. According to Remark 7.5.2 $\operatorname{Pr}(\tilde{\Delta}, \Delta) \simeq \operatorname{Pr}\left(\tilde{\Delta}^{\prime}, \Delta^{\prime}\right)$, where $\Delta^{\prime}=\phi\left(\Delta_{1}\right)$. It is easy to see that the embedding $\Delta^{\prime}=\phi\left(\Delta_{1}\right) \subset$ $\mathbb{P p}_{\mathrm{a}}(\Delta)-1$ is canonical. Now we can apply Theorem 7.5 and Remark 7.5.1 and obtain a contradiction as above.

In view of Theorems 9.1 and 9.2 one can expect that the rationality of a three-dimensional variety is equivalent to the triviality of Griffiths component $\mathrm{J}_{\mathrm{G}}(X)=0$. However this statement is wrong in general as the following Sarkisov's result shows. 
9.6. Theorem ([Sar82, Theorem 5.10]). There exists a smooth algebraic rationally connected non-rational threefold $X$ whose threedimensional integral cohomology group $H^{3}(X, \mathbb{Z})$ is trivial.

Proof. Let $\Delta_{0} \subset \mathbb{P}^{2}$ be an irreducible curve with at most ordinary double singular points whose normalization $\Delta$ has genus 1 . Assume that $\operatorname{deg} \Delta_{0}>12$. Let $\sigma:(S, \Delta) \rightarrow\left(\mathbb{P}^{2}, \Delta_{0}\right)$ be the minimal embedded resolution of singularities. Finally, let $\tilde{\pi}: \tilde{\Delta} \rightarrow \Delta$ be a nontrivial étale double cover. Then from Lemma 3.10 it follows that there exists a standard conic bundle $\pi: X \rightarrow S$ with discriminant divisor $\Delta$ such that the corresponding double cover is isomorphic to $\tilde{\pi}: \tilde{\Delta} \rightarrow \Delta$. It is easy to see that the curve $\Delta$ satisfies the condition $\left|4 K_{S}+\Delta\right| \neq$ $\varnothing$ and therefore $X$ is a non-rational variety by Theorem 12.2 below. On the other hand, by (3.6.3) we have $H^{3}(X, \mathbb{C})=0$ and since the discriminant curve of $\pi: X \rightarrow S$ connected, it follows that $H^{3}(X, \mathbb{Z})$ is torsion-free by (6.3.1). This proves the theorem.

\section{0. $\mathbb{Q}$-CONIC BUNDLES}

To study the local structure of $\mathbb{Q}$-conic bundles one needs the corresponding concept that works in the analytic category.

10.1. Definition. Let $(X, C)$ be an analytic germ of a threefold with terminal singularities along a reduced complete curve. We say that $(X, C)$ is a $\mathbb{Q}$-conic bundle germ if there is a contraction

$$
\pi:(X, C) \rightarrow(S, o)
$$

to a normal surface germ $(S, o)$ such that $C=\pi^{-1}(o)_{\text {red }}$ and the divisor $-K_{X}$ is $\pi$-ample.

Note that in this definition we assume neither $X$ is (analytically) $\mathbb{Q}$ factorial nor $\rho_{\mathrm{an}}(X / S)=1$. This is because these properties are not stable under passing from algebraic to analytic category, cf. Kaw88, $\S 1]$.

Q-conic bundle germs were studied in the series of papers [Pro97a], [Pro97b, Pro99a], Pro99b, MP08a, MP08b, MP09, MP11, [MP14], [MP16], [MP17. These works essentially use the ideas and techniques introduced by S. Mori Mor88 and developed in [KM92.

If $X$ has only Gorenstein (terminal) singularities along $C$, then, as in the standard conic bundle case, the base surface $S$ is smooth at $o$ and $\pi$ is a conic bundle, i.e. $X$ admits a local embedding to $\mathbb{P}^{2} \times S$ so that fibers of $\pi$ are conics [Cut88. In this case, the singularities of $X$ and the discriminant curve are partially described in Pro15a, Prop. 5.2]. From now on we assume that $X$ is not Gorenstein. 
Recall that the index of a $\mathbb{Q}$-Gorenstein singularity $(X, P)$ (or a $\mathbb{Q}$-Gorenstein variety $X)$ is called the smallest positive integer $m$ such that $m K_{X}$ is a Cartier divisor. For the classification of threedimensional terminal singularities and corresponding notations we refer to [Rei87, §6] and [KM98, Theorem 5.43].

If the total space $X$ has only singularities of index two and the base is smooth, then the description $\mathbb{Q}$-conic bundle germs is very explicit:

10.2. Theorem ([MP08a], Pro97b]). Let $\pi:(X, C) \rightarrow(S, o)$ be a $\mathbb{Q}$ conic bundle germ. Assume that $X$ is not Gorenstein, $(S, o)$ is smooth and the singularities of $X$ have indices $\leq 2$. Fix an isomorphism $(Z, o) \simeq\left(\mathbb{C}^{2}, 0\right)$. Then there is an embedding

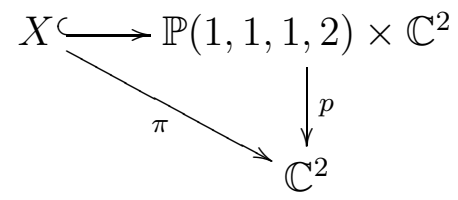

such that $X$ is given by two equations

$$
\begin{aligned}
& q_{1}\left(y_{1}, y_{2}, y_{3}\right)=\psi_{1}\left(y_{1}, \ldots, y_{4} ; u, v\right), \\
& q_{2}\left(y_{1}, y_{2}, y_{3}\right)=\psi_{2}\left(y_{1}, \ldots, y_{4} ; u, v\right),
\end{aligned}
$$

where $\psi_{i}$ and $q_{i}$ are quasihomogeneous polynomials in $y_{1}, \ldots, y_{4}$, quadratic with respect to the weight $\operatorname{wt}\left(y_{1}, \ldots, y_{4}\right)=(1,1,1,2)$ and $\psi_{i}\left(y_{1}, \ldots, y_{4} ; 0,0\right)=0$. The only non-Gorenstein point of $X$ is $(0,0,0,1 ; 0,0)$.

In the case where the base of a $\mathbb{Q}$-conic bundle is singular, we also have a complete classification:

10.3. Theorem ([MP08a], [MP08b]). Let $\pi:(X, C) \rightarrow(S, o)$ be a $\mathbb{Q}$-conic bundle germ. Assume that $(S, o)$ is singular. Then one of the following holds:

10.3.1. $(X, C)$ is biholomorphic to the quotient of $\mathbb{P}_{x}^{1} \times \mathbb{C}_{u, v}^{2}$ by the $\boldsymbol{\mu}_{m}$-action

$$
(x ; u, v) \longmapsto\left(\varepsilon x ; \varepsilon^{a} u, \varepsilon^{-a} v\right),
$$

where $\varepsilon$ is a primitive $m$-th root of unity and $\operatorname{gcd}(m, a)=\operatorname{gcd}(m, b)=$ 1. The singular locus of $X$ consists of two cyclic quotient singularities of types $\frac{1}{m}(1, a,-a)$ and $\frac{1}{m}(-1, a,-a)$. The base surface $\mathbb{C}^{2} / \boldsymbol{\mu}_{m}$ has a singularity of type $\mathrm{A}_{\mathrm{m}-1}$.

10.3.2. $(X, C)$ is biholomorphic to the quotient of the smooth $\mathbb{Q}$-conic bundle

$$
X^{\prime}=\left\{y_{1}^{2}+u y_{2}^{2}+v y_{3}^{2}=0\right\} \subset \mathbb{P}_{y_{1}, y_{2}, y_{3}}^{2} \times \mathbb{C}_{u, v}^{2} \longrightarrow \mathbb{C}_{u, v}^{2}
$$


by the $\boldsymbol{\mu}_{m}$-action

$$
\left(y_{1}, y_{2}, y_{3}, u, v\right) \longmapsto\left(\varepsilon^{a} y_{1}, \varepsilon^{-1} y_{2}, y_{3}, \varepsilon u, \varepsilon^{-1} v\right) .
$$

Here $m=2 a+1$ is odd and $\varepsilon$ is a primitive $m$-th root of unity. The singular locus of $X$ consists of two cyclic quotient singularities of types $\frac{1}{m}(a,-1,1)$ and $\frac{1}{m}(a+1,1,-1)$. The base surface $\mathbb{C}^{2} / \boldsymbol{\mu}_{m}$ has a singularity of type $\mathrm{A}_{\mathrm{m}-1}$.

10.3.3. $(X, C)$ is the quotient of an index two $\mathbb{Q}$-conic bundle of the form (10.2.2) with $q_{1}=y_{1}^{2}-y_{2}^{2}, q_{2}=y_{1} y_{2}-y_{3}^{2}$ by the $\boldsymbol{\mu}_{4}$-action

$$
\left(y_{1}, y_{2}, y_{3}, y_{4} ; u, v\right) \longmapsto\left(-\mathrm{i} y_{1}, \mathrm{i} y_{2},-y_{3}, \mathrm{i} y_{4} ; \mathrm{i} u,-\mathrm{i} v\right) \text {. }
$$

The base surface $(S, o)$ is Du Val of type $\mathrm{A}_{3}, X$ has a cyclic quotient singularity $P$ of type $\frac{1}{8}(5,1,3)$ and has no other singular points.

10.3.4. $(X, C)$ is a quotient of a Gorenstein conic bundle given by the following equation in $\mathbb{P}_{y_{1}, y_{2}, y_{3}}^{2} \times \mathbb{C}_{u, v}^{2}$

$$
y_{1}^{2}+y_{2}^{2}+\psi(u, v) y_{3}^{2}=0, \quad \psi(u, v) \in \mathbb{C}\left\{u^{2}, v^{2}, u v\right\}
$$

by the $\boldsymbol{\mu}_{2}$-action

$$
\left(y_{1}, y_{2}, y_{3} ; u, v\right) \longmapsto\left(-y_{1}, y_{2}, y_{3} ;-u,-v\right) .
$$

Here $\psi(u, v)$ has no multiple factors. In this case, $X$ has a unique singular point and it is of type $\mathrm{cA} / 2$ or $\mathrm{cAx} / 2$ and the base surface $(S, o)$ is Du Val of type $\mathrm{A}_{1}$.

10.3.5. $(X, C)$ is the quotient of an index two $\mathbb{Q}$-conic bundle of the form (10.2.2) with $q_{1}=y_{1}^{2}-y_{2}^{2}, q_{2}=y_{3}^{2}$ by the $\boldsymbol{\mu}_{2}$-action

$$
\left(y_{1}, y_{2}, y_{3}, y_{4} ; u, v\right) \longmapsto\left(y_{1},-y_{2}, y_{3},-y_{4} ;-u,-v\right) \text {. }
$$

The base $(S, o)$ is Du Val of type $\mathrm{A}_{1}$ and $X$ has a unique nonGorenstein point which is either a cyclic quotient singularity of type $\frac{1}{4}(1,1,-1)$ or a singularity of type $\mathrm{cAx} / 4$.

10.3.6. $(X, C)$ is the quotient of an index two $\mathbb{Q}$-conic bundle of the form (10.2.2) with $q_{1}=y_{1}^{2}-y_{3}^{2}, q_{2}=y_{2}^{2}-y_{3}^{2}$ by the $\boldsymbol{\mu}_{2}$-action

$$
\left(y_{1}, y_{2}, y_{3}, y_{4} ; u, v\right) \longmapsto\left(-y_{1},-y_{2}, y_{3},-y_{4} ;-u,-v\right) \text {. }
$$

The base $(S, o)$ is Du Val of type $\mathrm{A}_{1}$ and $X$ has a unique nonGorenstein point which is either a cyclic quotient singularity of type $\frac{1}{4}(1,1,-1)$ or a singularity of type $\mathrm{cAx} / 4$.

The central curve $C$ is irreducible except for the case 10.3.6 where $C$ has two irreducible component meeting at the non-Gorenstein point.

The main step in the proof of Theorem 10.3 is the following version of Reid's "general elephant conjecture". 
10.4. Theorem ([MP08a, MP09]). Let $\pi:(X, C) \rightarrow(S, o)$ be a $\mathbb{Q}$ conic bundle germ. Assume that $C$ is irreducible. Then a general member of the linear system $\left|-K_{X}\right|$ has only Du Val singularities.

The proof uses the techniques developed by Mori in the study of flipping extremal curve germs [Mor88, [KM92].

As an immediate consequence of Theorem 10.3 above we have the following

10.5. Theorem ([MP08a]). If $\pi: X \rightarrow S$ is a $\mathbb{Q}$-conic bundle, then $S$ can have at most Du Val singularities of type A.

If $(S \ni o)$ is of type $\mathrm{A}_{\mathrm{m}-1}$, then we say that the germ $(X, C)$ has topological index $\mathrm{m}$. In this case $(X, C)$ is a quotient of a $\mathbb{Q}$-conic bundle germ over a smooth base by $\boldsymbol{\mu}_{m}$ (see [MP08a, (2.4)], [Pro97b, Construction 1.9]).

10.6. Note that the condition that $X$ has terminal singularities is crucial in Theorem 10.5. In general (for contractions in the log terminal category), the singularities of the base are worse than canonical. It is known that the base $S$ of an arbitrary Mori fiber space $\pi: X \rightarrow S$ is a normal $\mathbb{Q}$-factorial variety with at worst log terminal singularities [KMM87, Lemma 5-1-5], [Fuj99.

10.6.1. Example. Consider the following action of $\boldsymbol{\mu}_{m}$ on $\mathbb{P}_{x}^{1} \times \mathbb{C}_{u, v}^{2}$ :

$$
(x ; u, v) \longmapsto\left(\varepsilon x ; \varepsilon u, \varepsilon^{m-2} v\right),
$$

where $m$ is odd and $\varepsilon$ is a primitive $m$-th root of unity. Let $X:=$ $\mathbb{P}^{1} \times \mathbb{C}^{2} / \boldsymbol{\mu}_{m}, S:=\mathbb{C}^{2} / \boldsymbol{\mu}_{m}$ and let $\pi: X \rightarrow S$ be the natural projection. Since $\boldsymbol{\mu}_{m}$ acts freely in codimension one, $-K_{X}$ is $f$-ample. Two fixed points on $\mathbb{P}^{1} \times \mathbb{C}^{2}$ gives two quotient singularities of $X$ which are terminal of type $\frac{1}{m}(-1,1,-2)$ and canonical Gorenstein of type $\frac{1}{m}(1,1,-2)$. In this case, $f$ is an extremal contraction in the category threefolds with canonical singularities. The base surface $S$ has a quotient singularity of type $\frac{1}{m}(1,-2)$ which is not canonical but $\delta$-log canonical with $\delta=(m+1) / 2 m$.

However, V. Shokurov conjectured that for any extremal $K$-negative contraction from threefold with only canonical singularities to a surface, the singularities of the base are 1/2-log canonical. More generally, there is the following conjecture posed by J. McKernan.

10.6.2. Conjecture. For fixed integer $n>0$ and a real number $\varepsilon>0$, there exists a constant $\delta=\delta(n, \varepsilon)>0$ such that the following holds: If $\pi: X \rightarrow S$ is an extremal contraction, where $X$ is a $\mathbb{Q}$-factorial $n$-dimensional variety with only $\varepsilon$-log terminal singularities, then the singularities of $S$ are $\delta$-log terminal. 
This conjecture in the toric case was proved by Alexeev and Borisov AB14. Partial results in general case were obtained in [Bir16b] and related to log adjunction (cf. [Sho13, Sho17]).

As a consequence of Theorem 10.3 we also have the following two facts.

10.7. Corollary. Let $\pi:(X, C) \rightarrow(S, o)$ be a $\mathbb{Q}$-conic bundle germ and let $\Delta \subset S$ be the discriminant curve. Then $\Delta$ is a Cartier divisor at o except for the cases [10.3.3, 10.3.5, 10.3.6.

10.8. Corollary. Let $\pi:(X, C) \rightarrow(S, o)$ be a $\mathbb{Q}$-conic bundle germ and let $\Delta \subset S$ be the discriminant curve. Then the following holds.

(i) $\Delta \not \supset o$ if and only if $(X, C)$ is of type 10.3.1.

(ii) If $\Delta \ni o$ and the pair $(S, \Delta)$ is plt at o, then $X$ is smooth near $C$ (and $S$ is smooth at $o$ ).

10.9. Results in the case of smooth base surface $S$ are not complete. However this case studied well under additional assumption that central curve $C$ is irreducible [MP09], MP11, MP14, [MP16], MP17]. The main strategy is as follows. Using a "good" member $D \in\left|-K_{X}\right|$ (Theorem 10.4) it is possible to analyze a general hyperplane section $H \subset X$ passing through $C$. Then the threefold $X$ can be viewed as the total space of a one-parameter deformation of $H$. If $H$ is normal, then its singularities are rational.

10.9.1. Example ([MP14]). Consider a normal surface germ $(H, C)$ along a curve $C \simeq \mathbb{P}^{1}$ whose minimal resolution $H_{\text {min }}$ have the following dual graph

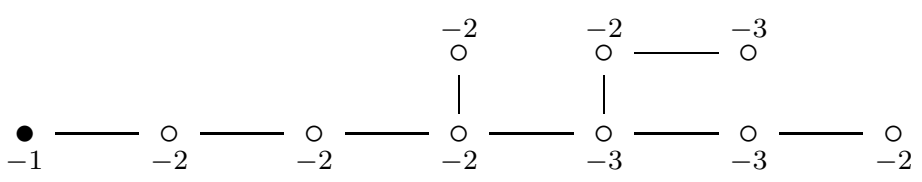

Here the white vertices correspond to exceptional rational curves and the black vertex corresponds to the curve $C$. The number attached to a vertex is the self-intersection of the corresponding curve. It is easy to see that the configuration of white vertices can be contracted to a rational singularity $H \ni P$ and the whole configuration is a fiber of a rational curve fibration $H_{\min } \rightarrow \Gamma$. Detailed computations KM92, 10.8] show that, locally, $H \ni P$ can be realized as a hyperplane section of a threefold terminal singularity

$$
(X, P) \simeq \mathbb{C}_{y_{1}, y_{2}, y_{4}}^{3} / \boldsymbol{\mu}_{5}(2,3,1)
$$

such that $H \supset C$, where

$$
C=\left\{y_{1}^{3}-y_{2}^{2}=y_{4}=0\right\} / \boldsymbol{\mu}_{5} .
$$


The deformations of $H$ are unobstructed, so a general one-parameter deformation of $H$ is a threefold $X \supset H$ with terminal singularity at $P$. We get a $\mathbb{Q}$-conic bundle $\pi:(X, C) \rightarrow(S, o)$ with smooth $S$.

The situation is more complicated in the case where $H$ is not normal. Let us give the simplest example.

10.9.2. Example ([MP11]). Let $\lambda_{1}, \lambda_{2} \in \mathbb{C}$ be some general constants, and let $X$ be the threefold given in $\mathbb{P}(1, a, m-a, m) \times \mathbb{C}_{t}$ by

$$
x_{1}^{2 m-2 a} x_{2}^{2}+x_{1}^{2 a} x_{3}^{2}+x_{2} x_{3} x_{4}+\left(\lambda_{1} x_{1}^{m}-x_{4}\right)\left(\lambda_{2} x_{1}^{m}-x_{4}\right) t=0 .
$$

Then a small analytic neighborhood of the curve

$$
C:=\left\{x_{2}=x_{3}=t=0\right\}
$$

is a $\mathbb{Q}$-conic bundle germ. The singular locus of $X$ near $C$ consists of a cyclic quotient singularity of type $\frac{1}{m}(1, a, m-a)$ and two (Gorenstein) ordinary double points. A general hyperplane section passing through $C$ is not normal.

In the sequel we need the following simple lemmas.

10.10. Lemma. Let $\pi:(X, C) \rightarrow(S, o)$ be a $\mathbb{Q}$-conic bundle germ over smooth base and let $\Delta$ be the discriminant curve. Assume that $X$ is singular and $\operatorname{mult}_{o}(\Delta)=2$. Take a standard model $\pi^{\bullet}: X^{\bullet} \rightarrow S^{\bullet}$ fitting in diagram (3.12.1) so that the relative Picard number $\rho\left(S^{\bullet} / S\right)$ is minimal. Let $\Delta^{\bullet} \subset S^{\bullet}$ be the discriminant curve. Then $\Delta^{\bullet}$ and the the exceptional divisor $\alpha^{-1}($ o) have no common components.

Proof. According to Corollary 3.3.6 we have $\Delta^{\bullet} \leq \alpha^{*} \Delta$ and $\alpha\left(\Delta^{\bullet}\right)=$ $\Delta$. The curve germ $o \in \Delta$ locally can be given by the equation $y^{2}-x^{n}=$ 0 and the inverse image $\alpha^{-1}(o)$ is a tree of rational curves. Let $\Delta^{\prime} \subset S^{\bullet}$ be the proper transform of $\Delta$. Clearly, $\Delta^{\prime} \subset \Delta^{\bullet}$. If $\Delta^{\prime}$ is singular at a point $P \in \alpha^{-1}(o)$, then $\Delta^{\prime} \cap \alpha^{-1}(o)=\{P\}$ and none of the components of $\alpha^{-1}(o)$ are contained in $\Delta^{\bullet}$ by Corollaries 3.3 .3 and 3.9.1. From now on we assume that $\Delta^{\prime}$ is smooth.

Suppose that $n$ is even. Then $\Delta$ has two smooth analytic branches at $o$ and their proper transforms $\Delta_{1}^{\bullet}$ and $\Delta_{2}^{\bullet}$ on $S^{\bullet}$ are disjoint. Furthermore, $\Delta_{i}^{\mathbf{i}}$ and $\Delta_{2}^{\mathbf{\bullet}}$ are connected by a chain of smooth rational curves $E_{1}, \ldots, E_{n} \subset \alpha^{-1}(o)$ and all components of $\alpha^{-1}(o)$ other than $E_{1}, \ldots, E_{n}$ are not contained in $\Delta^{\boldsymbol{\bullet}}$ by Corollary 3.9.1 and (3.8.2). Thus we may assume that $\Delta^{\bullet}$ contains some $E_{i}$. Again by (3.8.2)

$$
\Delta^{\bullet}=\Delta_{1}^{\bullet}+E_{1}+\cdots+E_{n}+\Delta_{2}^{\bullet}, \quad E_{i} \subset \Delta^{\bullet} \quad \forall i=1, \ldots, n .
$$

Clearly, $\alpha^{-1}(o)$ contains at least one $(-1)$-curve, say $E$, and $\Delta^{\bullet} \cdot E \leq 1$. Then by Proposition 8.5 we can blow down $E$ and get a standard model $X^{\prime} / S^{\prime}$ with smaller value $\rho\left(S^{\prime} / S\right)$. This contradicts our assumptions. 
Suppose that $n$ is odd. Then $\Delta^{\prime}$ is irreducible (and smooth) and $\Delta^{\prime} \cap \alpha^{-1}(o)$ is a single point, say $P$. By (3.8.2) the curves $\Delta^{\bullet}$ and $\alpha^{-1}(o)$ have no common components.

10.11. Lemma. Let $f:(X, C) \rightarrow(Z, o)$ be a $\mathbb{Q}$-conic bundle germ. Assume that there exists an effective Weil divisor $H$ on $X$ such that the pair $(X, H)$ is canonical and $K_{X}+H$ is numerically trivial. Then the divisor $K_{X}+H$ is Cartier.

Proof. Assume the converse, i.e. $K_{X}+H$ is not Cartier at some point $P \in C \subset X$ of index $m$. Since the point $P \in X$ is terminal, $k K_{X}+H$ is a Cartier divisor in a neighborhood of $P$ for some $k$ (see Kaw88, Corollary 5.2]). According to our assumption $k \not \equiv 1 \bmod m$. In particular, $m>1$. We may assume that $1<k \leq m$.

Assume that $H \ni P$. It follows from the main result of [Kaw93] that there exists an exceptional divisor $E$ over $P \in X$ with discrepancy $a(E, X, 0)=1 / m$. Since $k K_{X}+H$ is a Cartier divisor at $P$,

$$
-k a(E, X, 0)+\operatorname{mult}_{E}(H)=-k / m+\operatorname{mult}_{E}(H) \in \mathbb{Z} .
$$

Hence, $\operatorname{mult}_{E}(H) \geq k / m>1 / m$ and

$$
a(E, X, H)=a(E, X, 0)-\operatorname{mult}_{E}(H) \leq 1 / m-k / m<0 .
$$

This contradicts canonicity of the pair $(X, H)$. Thus, $H$ does not contain $P$.

Clearly, the point $P$ must be non-Gorenstein [Kaw88, Lemma 5.1]. Since the pair $(X, H)$ is canonical, it is easy to see from the main result of [Kaw93] that $P \notin H$. Since $\operatorname{Pic}(X) \simeq H^{2}(C, \mathbb{Z})$ and $K_{X}+H \equiv 0$, the divisor $K_{X}+H$ is a torsion element in the Weil divisor class group and it defines a non-trivial étale in codimension one cover of $(X, C)$ (see [MP08a, Corollaries 2.3.1 and 2.7.1]). In particular, $(Z, o)$ is a singular point. For any component $C_{i} \subset C$ we have

$$
H \cdot C_{i}=-K_{X} \cdot C_{i}<1
$$

(see [MP08a, Lemma 2.8]). Therefore $H$ is not a Cartier divisor at some point $P_{1} \in C \subset X, P_{1} \neq P$. Thus $X$ has at least two nonGorenstein points. By Theorem 10.3 the germ $(X, C)$ is of type 10.3 .1 or 10.3.2. Then $C$ is irreducible and $H \cap C=\left\{P_{1}\right\}$. The torsion subgroup $\mathrm{Cl}(X)_{\text {tors }}$ of the Weil divisor class group $\mathrm{Cl}(X)$ is cyclic [MP08a, Corollary 2.7.1]. One can see from the constructions that in both cases 10.3 .1 and 10.3 .2 the restrictions $\mathrm{Cl}(X)_{\text {tors }} \rightarrow \mathrm{Cl}(X, P)$ and $\mathrm{Cl}(X)_{\text {tors }} \rightarrow \mathrm{Cl}\left(X, P_{1}\right)$ to the local class groups are isomorphisms. In particular, this means that $K_{X}+H$ is not a Cartier divisor at $P_{1}$. According to the discussions in the beginning of the proof we have $H \not \supset P_{1}$, a contradiction. 


\section{Examples of SARKisov LinkS ON $\mathbb{Q}$-CONIC BUNDLES}

In this section we consider Sarkisov links on non-Gorenstein $\mathbb{Q}$-conic bundles. Such links are elementary steps in the decomposition of birational transformations of conic bundles (see $\S$ 12). For applications it would be very useful to have their classification or at least substantive theorems, analogous to Theorem 5.1, describing their structure. Since very little is known in this direction, we shall restrict ourselves to a number of examples.

In this section we consider some examples of Sarkisov links on nonGorenstein $\mathbb{Q}$-conic bundles. We use the following natural construction.

11.1. Construction. Let $\pi:(X, C) \rightarrow(S, o)$ be a $\mathbb{Q}$-conic bundle germ, let $C_{1}, \ldots, C_{r}$ be irreducible components of $C$, and let $P \in C \subset$ $X$ be a point of index $m_{1}>1$. We consider only the cases described in Theorems 10.2 or 10.3. In all these cases all the curves $C_{i}$ pass through $P$. Then for any $i=1, \ldots, r$ we have

$$
-K_{X} \cdot C_{i}= \begin{cases}1 / 2 & \text { in the cases 10.2, } \\ 1 & 10.3 .3,10.3 .5,10.3 .6 \\ 2 / m_{1} & \text { in the case 10.3.4, } \\ 1 / m_{1} & \text { in the case 10.3.1, }\end{cases}
$$

These equalities can be checked directly or deduced from MP08a, Lemma 2.8] and [Mor88, (2.3), (4.9)]. According to [Kaw93, Hay99, Hay00 there exists an extraction $p: Z \rightarrow X$ in Mori category with exceptional divisor $E$ such that $p(E)=P$ and the discrepancy of $E$ equals $1 / m_{1}$. Let $\tilde{C}_{i} \subset Z$ be the proper transform of $C_{i}$. Then

$$
K_{Z} \cdot \tilde{C}_{i}=K_{X} \cdot C_{i}+\frac{1}{m_{1}} E \cdot \tilde{C}_{i}
$$

Assume that

$$
K_{Z} \cdot \tilde{C}_{i} \leq 0
$$

(thus $Z$ is the central object of the corresponding link [SC11]). This inequality holds and can be checked directly in many cases. Then there exists a flop or a flip along $\tilde{C}_{i}$ and we can run the MMP on $Z$ over $S$ in the direction different from the contraction to $X$. After some number of flops and flips

$$
\chi: Z \rightarrow Z_{1}
$$

we get a non-small Mori extremal contraction. There are two possibilities: either this contraction $q: Z_{1} \rightarrow X_{1}$ is divisorial or it is a $\mathbb{Q}$-conic bundle $\pi_{1}: Z_{1}=X_{1} \rightarrow S$. In other words, we obtain a link of type II 
or of type I, respectively (see 4.1). Moreover, for a type II link, the morphism $q$ contracts the proper transform of $E$. Thus $X$ and $X_{1}$ are isomorphic in codimension one. Since divisors $-K_{X}$ and $-K_{X_{1}}$ are ample over $S$, the varieties $X$ and $X_{1}$ are, in fact, isomorphic. If in the case of a type $\mathbf{I}$ link the base $(S, o)$ is singular, then the morphism $\alpha: S_{1} \rightarrow S$ must be a crepant contraction according to the following fact.

11.2. Proposition ([Mor85]). Let $S^{\prime} \rightarrow S^{\prime \prime}$ be a proper birational contraction between surfaces with Du Val singularities. Then $\alpha$ can be decomposed as a sequence $\alpha_{i}: S_{i} \rightarrow S_{i+1}$, where each $\alpha_{i}$ is either a crepant contraction or a weighted blowup with weights $\left(1, n_{i}\right)$ of a smooth point.

11.3. Recall that Shokurov's difficulty $\mathrm{d}_{\amalg}(V)$ of a variety $V$ with terminal singularities is defined as the number of exceptional divisors on $V$ with discrepancy $<1$ (see [Sho86, Definition 2.15]). It is known that this number is well-defined and finite. Moreover, in the threedimensional case, it is strictly decreasing under flips [Sho86, Corollary 2.16]. If $V \ni P$ is a terminal cyclic quotient of index $m$, then $\mathrm{d}_{\amalg}(V \ni$ $P)=m-1$. Thus, in our case (11.1.4) we have

$$
\mathrm{d}_{\amalg}\left(Z_{1}\right) \geq \mathrm{d}_{\amalg}(Z)
$$

and the inequality is strict if $\chi$ contains at least one flip.

Below we consider explicit examples. We use the notation of Construction 11.1.

11.4. Example. Let $(X, C) \subset \mathbb{P}(1,1,1,2)$ be the index two $\mathbb{Q}$-conic bundle germ given by

$$
\left\{\begin{array}{l}
y_{1}^{2}-y_{2}^{2}=u y_{4} \\
y_{1} y_{2}-y_{3}^{2}=v y_{4} .
\end{array}\right.
$$

(see (10.2.2) $)$ and let $\pi:(X, C) \rightarrow(S, o)=\left(\mathbb{C}^{2}, 0\right)$ be the corresponding contraction. Then $X$ has a unique singular point $P=$ $(0,0,0,1 ; 0,0)$ which is terminal quotient of type $\frac{1}{2}(1,1,1)$. The central curve has four components $C_{1}, \ldots, C_{4}$. All of them pass through $P$ and they do not meet each other elsewhere. The discriminant curve is given by $\left(u^{2}+4 v^{2}\right) u=0$. Let $p: Z \rightarrow X$ be the blowup of $P$, let $E$ be the exceptional divisor, and let $\tilde{C}_{i} \subset Z$ be the proper transform of $C_{i}$. Then $Z$ is smooth, $E \simeq \mathbb{P}^{2}$, and $\mathscr{O}_{E}(E) \simeq \mathscr{O}_{\mathbb{P}^{2}}(-2)$ (cf. [Mor82]). Furthermore, the curves $\tilde{C}_{1}, \ldots \tilde{C}_{4}$ are disjoint. By (11.1.1) and (11.1.2) we have $K_{Z} \cdot \tilde{C}_{i}=0$. There exists a flop $\chi: Z \rightarrow X_{1}$ with center $\tilde{C}$ which is the simplest Atiyah-Kulikov flop along each curve $\tilde{C}_{i}$. Since $-K_{X_{1}}$ is nef, there exists a Mori extremal contraction $\pi_{1}: X_{1} \rightarrow S_{1}$ 
over $S$. The restriction $\chi_{E}: E \rightarrow E_{1} \subset X_{1}$ is the inverse to the blowup of four points $E \cap \tilde{C}_{i}$. Hence, the proper transform $E_{1} \subset X_{1}$ of $E$ is a del Pezzo surface of degree 5. Thus $\pi_{1}: X_{1} \rightarrow S_{1}$ is a standard conic bundle (see e.g. [Mor82]). Then we get a type I link, where $S_{1}$ is a smooth surface and $\alpha: S_{1} \rightarrow S$ is the blowup of $o$. The discriminant curve $\Delta_{1}$ of $\pi_{1}$ is the proper transform of $\Delta$ (and so $\Delta_{1}$ has three smooth irreducible branches near $\left.\alpha^{-1}(o)\right)$.

11.5. Example. Let $\pi:\left(X, C \simeq \mathbb{P}^{1}\right) \rightarrow(S, o)$ be the toric $\mathbb{Q}$-conic bundle germ as in 10.3.1. In this case the discriminant curve is empty. Consider the Kawamata weighted blowup $p: Z \rightarrow X$ of the point $P \in X$ of type $\frac{1}{m}(1, a, m-a)$ (see [Kaw96]). It is easy to see that $Z$ has on $E$ two terminal quotient singularities of types

$$
\frac{1}{a}(1,-m, m) \quad \text { and } \quad \frac{1}{m-a}(1, a,-m),
$$

and $E \cap \tilde{C}$ is a smooth point of $Z$. By (11.1.1) and (11.1.2) we have $K_{Z} \cdot \tilde{C}=-1 / m$. Hence $\tilde{C}$ is a flipping curve. Run the MMP on $Z$ over $S$ as in (11.1.4). We have

$$
\mathrm{d}_{\amalg}(Z)=2 m-3, \quad \mathrm{~d}_{\amalg}\left(Z_{1}\right) \leq 2 m-4 .
$$

If we are in the situation of type II link, then $\pi_{1}: X_{1} \rightarrow S$ must be $\mathbb{Q}$-conic bundle of the same type as $\pi$ (because its discriminant divisor is trivial). On the other hand, $\mathrm{d}_{\amalg}\left(X_{1}\right) \leq \mathrm{d}_{\amalg}\left(Z_{1}\right)+1 \leq 2 m-3$, so $\mathrm{d}_{\amalg}\left(X_{1}\right)<\mathrm{d}_{\amalg}(X)$, a contradiction. Therefore, we have a type $\mathbf{I}$ link and $\alpha: S_{1} \rightarrow S$ is a crepant blowup of $o \in S$ (see Proposition 11.2). Thus $S_{1}$ has Du Val singularities of types $\mathrm{A}_{\mathrm{m}_{1}-1}$ and $\mathrm{A}_{\mathrm{m}_{2}-1}$ with $m_{1}+$ $m_{2}=m$. We may assume that $m_{1} \geq m_{2}$ (it is possible that $m_{2}=1$ ). The discriminant divisor of $\pi_{1}$ is trivial, so $\pi_{1}$ is also of type 10.3.1 More precisely, $\pi_{1}$ has two (or one) germs of type 10.3.1 which are quotients by $\boldsymbol{\mu}_{m_{1}}$ and $\boldsymbol{\mu}_{m_{2}}$. In this case,

$$
\mathrm{d}_{\amalg}\left(X_{1}\right)=2 m_{1}-2+2 m_{2}-2=2 m-4 .
$$

Thus in (11.5.2) the equality holds. This implies that $\chi$ is a single flip and so $X_{1}$ has singularities of types (11.5.1). This means that up to permutation we have $m_{1}=a$ and $m_{2}=m-a$.

11.6. Example. Let $\pi:\left(X, C \simeq \mathbb{P}^{1}\right) \rightarrow(S, o)$ be the $\mathbb{Q}$-conic bundle germ as in 10.3.2. In this case the discriminant curve $\Delta$ is given by $\{u v=0\} / \boldsymbol{\mu}_{m}$. In particular, the pair $(S, \Delta)$ is log canonical. Consider the Kawamata weighted blowup $p: Z \rightarrow X$ of the point $P \in X$ of type $\frac{1}{m}(a+1,1,-1)=\frac{1}{m}(1,2, m-2)$. Then $E \cap \tilde{C}$ is a singularity of type $\frac{1}{2}(1,1,1)$ on $Z$. By (11.1.1) and (11.1.2) we have $K_{Z} \cdot \tilde{C}=$ $-1 / 2 m$. Running the MMP as in Construction 11.1 we obtain a type $\mathbf{I}$ Sarkisov link, where $\pi_{1}$ is a $\mathbb{Q}$-conic bundle and $\alpha: S_{1} \rightarrow S$ is a 
crepant contraction. For the discriminant curve $\Delta_{1} \subset S_{1}$ we have $\Delta_{1}=$ $\alpha^{*}(\Delta)_{\text {red }}$ (see Corollary 10.8) and the pair $\left(S_{1}, \Delta_{1}\right)$ is lc. This implies that $\pi_{1}$ cannot contain germs of type 10.3.1. The same computations with difficulty as in Example 11.5 show that $\chi$ is a single flip and so $X_{1}$ contains a singularity of type $\frac{1}{m-1}(1,2,-2)$. Therefore, $S_{1}$ has two $\mathrm{Du}$ Val points of types $\mathrm{A}_{\mathrm{m}-3}$ and $\mathrm{A}_{1}$ and over $\mathrm{A}_{\mathrm{m}-3}$ we have a germ of type 10.3.2 of index $m-2$. Then by the classification 10.3 for the germ over $\mathrm{A}_{1}$ there is only one possibility: case 10.3.4 with $\psi=u v$.

11.7. Example. Consider a $\mathbb{Q}$-conic bundle germ $\pi:(X, C) \rightarrow(S, o)$ of type 10.3 .3 which is the $\boldsymbol{\mu}_{4}$-quotient of $X^{\prime} \subset \mathbb{P}(1,1,1,2)$ defined by (11.4.1). The discriminant curve $\Delta$ is given by $\left\{\left(u^{2}+4 v^{2}\right) u=0\right\} / \boldsymbol{\mu}_{4}$ on $S=\mathbb{C}^{2} / \boldsymbol{\mu}_{4}(1,-1)$. One can see that the dual graph of the minimal resolution of $(S \supset \Delta \ni o)$ has the following form

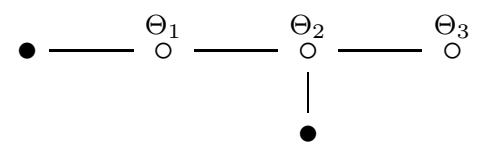

where the vertices $\circ$ correspond to (crepant) exceptional divisors over $o$ and the vertices $\bullet$ correspond to the components of $\Delta$. Consider the Kawamata weighted blowup $p: Z \rightarrow X$ of the point $P \in X$ of type $\frac{1}{8}(5,1,3)$. Then $Z$ has two cyclic quotient points $P_{1}$ and $P_{2}$ of types $\frac{1}{5}(-3,1,3)$ and $\frac{1}{3}(2,1,1)$. The curve $\tilde{C}$ passes through $P_{1}$. Using (11.1.1) and (11.1.2) we can compute $K_{Z} \cdot \tilde{C}<0$. Thus there is a flip along $\tilde{C}$. We have $\mathrm{d}_{\amalg}(Z)=6$ and $\mathrm{d}_{\amalg}\left(Z_{1}\right) \leq 5$. As above we get a type I link, where $\alpha: S_{1} \rightarrow S$ is a crepant contraction. Let $\Delta_{1} \subset S_{1}$ be the discriminant curve. If $\alpha$ is the blowup of $\Theta_{2}$, then $S_{1}$ has two points, say $o_{1}$ and $o_{2}$, of type $A_{1}$. By Corollary 10.8 we have $\alpha^{-1}(o) \subset \Delta_{1}$. But then the pair $\left(S_{1}, \Delta_{1}\right)$ is plt at one the $o_{i}$. This is impossible again by Corollary 10.8. Thus $S_{1}$ has a point $o_{1}$, of type $\mathrm{A}_{2}$. Then by the classification 10.3 the germ over $o_{2}$ is of type 10.3 .2 and so the pair $\left(S_{1}, \Delta_{1}\right)$ is lc at $o_{1}$. Therefore, $\alpha^{-1}(o) \not \subset \Delta_{1}$ and $\alpha$ is the extraction of $\Theta_{3}$.

11.8. Example ([Avi14]). Consider a $\mathbb{Q}$-conic bundle germ $\pi$ : $(X, C) \rightarrow(S, o)$ such that $(S, o)$ is smooth and the discriminant curve $\Delta$ has a node at $o$. Denote $U:=S \backslash\{o\}$ and $V:=X \backslash C=\pi^{-1}(U)$. Let $j: U \hookrightarrow S$ be the embedding. The sheaf $\mathscr{E}_{0}:=\pi_{*} \mathscr{O}_{V}\left(-K_{X}\right)$ is locally free on $U$. It has a unique extension to $S$ as a locally free sheaf. Indeed, the sheaf $\left(j_{*} \mathscr{E}_{0}\right)^{\vee \vee}$ is reflexive and therefore it is locally free. Clearly, $V$ is embedded to $\mathbb{P}_{U}\left(\mathscr{E}_{0}\right) \subset \mathbb{P}_{S}(\mathscr{E})$. Let $X^{\prime} \subset \mathbb{P}_{S}(\mathscr{E})$ be the closure of $V$. We may assume that $(S, o)$ is an open (analytic) subset of $\mathbb{C}_{u, v}^{2}$ and $\Delta$ is given by $u v=0$. 
Now assume that $X$ is singular. Then easy computations show that $X^{\prime}$ can be given locally by the equation

$$
u v x_{0}^{2}+x_{1}^{2}+x_{2}^{2}=0
$$

in $\mathbb{P}_{S}(\mathscr{E})=\mathbb{P}_{x_{0}, x_{1}, x_{2}}^{2} \times \mathbb{C}_{u, v}^{2}$. In particular, the projection $\pi^{\prime}: X^{\prime} \rightarrow S$ is flat and $X^{\prime}$ has a unique singular point $P \in X^{\prime}$ which is a node. On the other hand the varieties $X^{\prime}$ and $X$ are isomorphic in codimension one over $S$ and their anti-canonical divisors are relatively ample. Hence the varieties $X^{\prime}$ and $X$ are isomorphic over $S$ and we can identify them. In particular, $X$ can be embedded to $\mathbb{P}_{S}(\mathscr{E})$.

Let $p: Z \rightarrow X$ be the blowup of $P$. Then $Z$ is smooth and $-K_{Z}$ is nef and big over $S$. There exists a flop $Z \rightarrow X_{1}$ with center the proper transform of $C$ and on $X_{1}$ there exists a Mori extremal contraction $\pi_{1}: X_{1} \rightarrow S_{1}$ over $S$. Since $X_{1}$ is smooth, so $S_{1}$ is. Thus we obtain a type I Sarkisov link, where $\alpha: S_{1} \rightarrow S$ is the blowup of $o$. Moreover, the exceptional curve $\alpha^{-1}(o)$ is not contained in the discriminant divisor $\Delta_{1} \subset S_{1}$ of $\pi_{1}$ (otherwise we are in the situation of Proposition 8.5)(iii) and $X$ is smooth). Therefore, $\Delta_{1}$ is the proper transform of $\Delta$.

11.8.1. Corollary. Let $\pi:(X, C) \rightarrow(S, o)$ be a $\mathbb{Q}$-conic bundle germ and let $\Delta$ be the discriminant curve. Assume that the pair $(S, \Delta)$ is lc but not plt at o. Then either $\pi$ is a standard conic bundle, or $\pi$ is as in Example 11.8, or as in 10.3.2 or 10.3.4 with $\psi=u v$.

Proof. By Theorem 10.5 the germ $(S, o)$ is the quotient of a smooth germ $\left(S^{\prime}, o^{\prime}\right)$ by $\boldsymbol{\mu}_{m}, m \geq 1$. Let $X^{\prime}$ be the normalization of the fiber product $X \times{ }_{S} S^{\prime}$ (see [MP08a, (2.4)], [Pro97b, Construction 1.9]). Then the projection $\pi^{\prime}: X^{\prime} \rightarrow S^{\prime}$ is a $\mathbb{Q}$-conic bundle germ over smooth base. By Example 11.8 the variety $X^{\prime}$ is Gorenstein. Then the assertion follows from [Pro97b, Theorem 2.4].

11.8.2. Corollary. Let $\pi:(X, C) \rightarrow(S, o)$ be a $\mathbb{Q}$-conic bundle germ such that its discriminant curve $\Delta$ is non-empty and smooth at $o$. Then both $X$ and $S$ are smooth and $\pi$ is a standard conic bundle.

Proof. By Theorem 10.3 the base $(S, o)$ is either smooth or Du Val of type $\mathrm{A}_{1}$ or $\mathrm{A}_{3}$. In these cases the pair $(S, \Delta)$ must be lc because the curve $\Delta$ is smooth. Then the assertion follows from Corollaries 11.8 .1 and 10.8 .

A. Avilov Avi14 used Sarkisov links to show the existence of standard models for three-dimensional $\mathbb{Q}$-conic bundles over a non-closed field of characteristic 0 (cf. Theorem 3.12). His construction is canonical and works in the category of $G$-varieties. In particular, he used the following easy observation. 
11.9. Lemma ([Avi14, Lemma 3]). Let $\pi:(X, C) \rightarrow(S, o)$ be a $\mathbb{Q}$ conic bundle germ with singular $(S, o)$. Then there exists a Sarkisov link of type $\mathbf{I}$, where $\alpha: S_{1} \rightarrow S$ is a crepant blowup of o.

Outline of the proof. Take a linear system $\mathscr{L}$ of hyperplane sections of $S$ passing trough $o$ and put $\mathscr{M}:=\pi^{*} \mathscr{L}$. Take $c$ so that the pair $(X, c \mathscr{M})$ is maximally canonical (in other words, $c=c(X, \mathscr{M})$ is the canonical threshold of $(X, \mathscr{M}))$. Then, as in 4.5, there exists a logcrepant extremal blowup $p:\left(Z, c \mathscr{M}_{Z}\right) \rightarrow(X, c \mathscr{M})$. Here $\rho(Z / S)=2$ and

$$
K_{Z}+c \mathscr{M}_{Z} \equiv p^{*}\left(K_{X}+c \mathscr{M}\right) .
$$

Moreover, $p$ is a divisorial contraction, and the variety $Z$ is $\mathbb{Q}$-factorial and has only terminal singularities. Then we run the $\left(K_{Z}+c \mathscr{M}_{Z}\right)$ MMP over $S$. Since $\operatorname{dim} S=2$, we obtain a link of type I. By Proposition 11.2 the contraction $\alpha: S_{1} \rightarrow S$ is a crepant blowup.

Avilov's proof of the existence of standard models is based on (weak) Sarkisov program and essentially uses Theorem 10.4. In fact, since the number of crepant divisors on $S$ is finite, any sequence of transformations as in Lemma 11.9 terminates. Therefore, applying such transformations one can resolve the singularities of the base.

\section{Birational transformations of $\mathbb{Q}$-CONiC BUNDLES, I}

Below we basically follow Iskovskikh's paper [Isk96b] with some improvements.

12.1. Theorem. Let $\pi: X \rightarrow S$ be a $\mathbb{Q}$-conic bundle over a projective rational surface $S$. Assume that there exist another Mori fiber space $\pi^{\sharp}: X^{\sharp} \rightarrow S^{\sharp}$ and a birational map

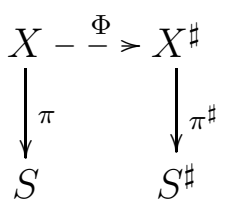

which cannot be completed to a commutative square. Then there exists the following commutative diagram

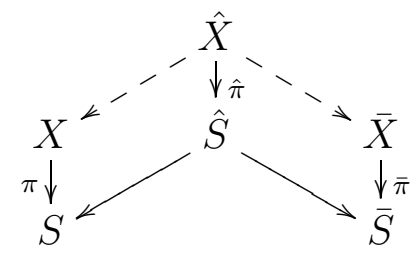

where $\hat{X} / \hat{S}$ and $\bar{X} / \bar{S}$ are $\mathbb{Q}$-conic bundles, $\hat{X} / \hat{S} \rightarrow X / S$ is a sequence of Sarkisov links of types I or II, $\hat{X} / \hat{S} \rightarrow \bar{X} / \bar{S}$ is a sequence of 
Sarkisov links of types III, and the contractions $\hat{S} \rightarrow S$ and $\hat{S} \rightarrow \bar{S}$ are described in Proposition 11.2. Furthermore, let $\bar{\Delta} \subset \bar{S}$ be the discriminant curve of $\bar{\pi}: \bar{X} \rightarrow \bar{S}$. Then one of the following holds:

(i) $\rho(\bar{S})=1$ and the divisor $-\left(4 K_{\bar{S}}+\bar{\Delta}\right)$ is ample;

(ii) $\rho(\bar{S})=2$ and there is a base point free pencil of rational curves $\overline{\mathscr{L}}$ such that

$$
2 \leq \overline{\mathscr{L}} \cdot \bar{\Delta}<-4 \overline{\mathscr{L}} \cdot K_{\bar{S}}=8
$$

Moreover, if $\pi$ is a standard conic bundle, then we have

$$
\mathrm{p}_{\mathrm{a}}(\hat{\Delta})=\mathrm{p}_{\mathrm{a}}(\Delta) \leq \mathrm{p}_{\mathrm{a}}(\bar{\Delta}) .
$$

Proof. We apply Sarkisov program (see Theorem 4.2). First, we untwist the maximal singularities. This means that we apply a sequence of type $\mathbf{I}$ and II links as explained in 4.5 :

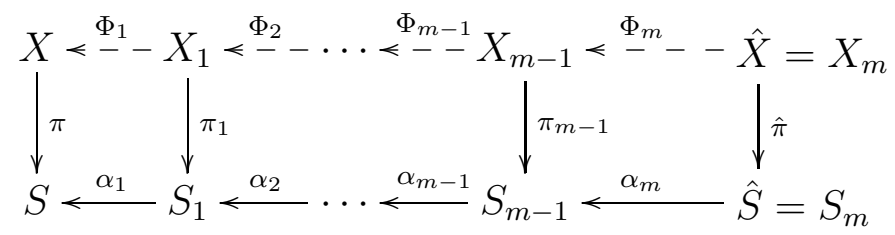

Here $\alpha_{i}$ are birational contractions or isomorphisms and each of the squares $\left(\Phi_{i}, \alpha_{i}\right)$ is commutative. Moreover, the proper transforms $\mathscr{H}_{X_{i}}$ of the linear system $\mathscr{H}_{X}$ have the form $\mathscr{H}_{X_{i}}=-\mu K_{X_{i}}+\pi_{i}^{*} A_{i}$, where $A_{i}$ is a $\mathbb{Q}$-divisor on $S_{i}$. On the last step, the linear system $\mathscr{H}_{\hat{X}}$ has no maximal singularities.

Next, we follow the step 4.6 of the Sarkisov program. The divisor $\hat{A}$ is not nef by the Noether-Fano inequality (Theorem 4.4), where $\hat{A}$, as usual, satisfies the relation

$$
\mathscr{H}_{\hat{X}} \equiv-\mu K_{\hat{X}}+\hat{\pi}^{*} \hat{A} .
$$

Therefore, there is a type III or type IV link $\Phi_{m+1}: \hat{X} / \hat{S} \rightarrow$ $X_{m+1} / S_{m+1}$. Assume that this link is of type III with birational contraction $\alpha_{m+1}: \hat{S} \rightarrow S_{m+1}$. Then $\pi_{m+1}: X_{m+1} \rightarrow \hat{S}_{m+1}$ satisfies the same conditions as $\hat{\pi}: \hat{X} \rightarrow \hat{S}$ and the procedure may be repeated:

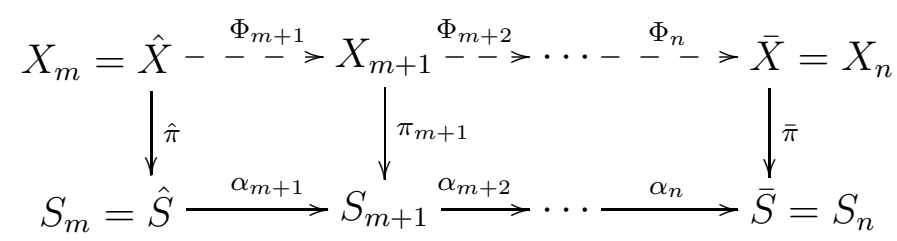

such that all the $\alpha_{m+i}, i=1, \ldots, n-m$, are birational contractions, $\rho\left(S_{m+i} / S_{m+i+1}\right)=1$, each of the squares $\left(\Phi_{m+i}, \alpha_{m+i}\right)$ is commutative, the proper transforms of $\mathscr{H}_{\hat{X}}$ on $X_{m+i}$ have the form $\mathscr{H}_{X_{m+i}}=$ 
$-\mu K_{X_{m+i}}+\pi_{m+i}^{*} A_{m+i}$, where $A_{m+i}$ is some non-nef $\mathbb{Q}$-divisor on $S_{m+i}$, and $\mathscr{H}_{X_{m+i}}$ has no maximal singularities.

After a finite number of steps we arrive at a link $\bar{\Phi}: \bar{X} / \bar{S} \rightarrow X^{b} / S^{b}$ of type III with a non-birational contraction $\delta=\bar{\alpha}: \bar{S} \rightarrow T=\bar{S}_{1}$ or of type IV. As above, for the proper transform $\mathscr{H}_{\bar{X}}$ of $\mathscr{H}$, we can write

$$
\mathscr{H}_{\bar{X}} \equiv-\mu K_{\bar{X}}+\bar{\pi}^{*} \bar{A},
$$

where $\bar{A}$ is not nef by the Noether-Fano inequality (Theorem 4.4). By the projection formula and (3.11.1) we have

$$
\Gamma:=\pi_{*}\left(\mathscr{H}_{\bar{X}}^{2}\right) \equiv 4 \mu \bar{A}-\mu^{2}\left(4 K_{\bar{S}}+\bar{\Delta}\right)
$$

and this cycle is an effective non-zero integral Weil divisor on $\bar{S}$. By the Noether-Fano inequality (Theorem 4.4) the divisor $-\bar{A}$ is not nef. We have two possibilities:

12.1.9. The link $\bar{\Phi}: \bar{X} / \bar{S} \rightarrow X^{\mathrm{b}} / S^{\mathrm{b}}$ is of type IV and $T$ is a point. Then $\rho(\bar{S})=1$. In this case, the divisors $-\bar{A},-K_{\bar{S}}$, and $-\left(4 K_{\bar{S}}+\bar{\Delta}\right)$ are ample by (12.1.8).

12.1.10. The link $\bar{\Phi}: \bar{X} / \bar{S} \rightarrow X^{b} / S^{b}$ is of type III (resp. IV), where in the notation of 4.1, the variety $S_{1}$ (resp. $T$ ) is a curve. Then this curve must be smooth rational and $\rho(\bar{S})=2$. Thus we have an extremal contraction $\delta: \bar{S} \rightarrow \mathbb{P}^{1}$. Let $\overline{\mathscr{L}}$ be the pencil of fibers. Then $\bar{A} \cdot \overline{\mathscr{L}}<0$ by the construction of the link $\bar{\Phi}$ (see 4.6). Further, $\left(4 K_{\bar{S}}+\bar{\Delta}\right) \cdot \overline{\mathscr{L}}<0$ by (12.1.8). This proves (12.1.3).

To prove the last equality, consider a standard conic bundle $\pi^{\bullet}$ : $X^{\bullet} \rightarrow S^{\bullet}$ that is birationally equivalent to $\hat{\pi}: \hat{X} \rightarrow \hat{S}$, as in Theorem 3.12. Let $\Delta^{\bullet} \subset S^{\bullet}$ be the corresponding discriminant curve. Then by Lemma 8.3 ,

$$
\mathrm{p}_{\mathrm{a}}\left(\Delta^{\bullet}\right) \leq \mathrm{p}_{\mathrm{a}}(\hat{\Delta}) \leq \mathrm{p}_{\mathrm{a}}(\Delta) \leq \mathrm{p}_{\mathrm{a}}(\bar{\Delta}) .
$$

But $\mathrm{p}_{\mathrm{a}}(\Delta)=\mathrm{p}_{\mathrm{a}}\left(\Delta^{\bullet}\right)$ by Lemma 8.4. This completes the proof of the theorem.

As an easy consequence of Theorem 12.1 we obtain the following.

12.2. Theorem (Sarkisov's theorem [Sar81]). Let $\pi: X \rightarrow S$ be a standard conic bundle over a rational surface $S$ and let $\Delta$ be the discriminant curve. Assume that $\left|4 K_{S}+\Delta\right| \neq \varnothing$. Then any birational map $X \rightarrow X^{\sharp}$ to another Mori fiber space $\pi^{\sharp}: X^{\sharp} \rightarrow S^{\sharp}$ is fiberwise (in particular, $\pi: X \rightarrow S$ is birationally rigid).

Note that the condition $\left|4 K_{S}+\Delta\right| \neq \varnothing$ in terms of effective threshold is equivalent to et $(S, \Delta) \geq 4$ (see 8.7). 
Proof. Suppose that $4 K_{S}+\Delta$ is effective. Then by Lemma 8.4 the divisor $4 K_{S} \bullet+\Delta^{\bullet}$ on the standard model $\pi^{\bullet}: X^{\bullet} \rightarrow S^{\bullet}$ is also effective (see Theorem 3.12). Its images under the birational contraction $\hat{S} \rightarrow \bar{S}$ is also an effective $\mathbb{Q}$-divisor. This is a contradiction to Theorem 12.1](i) and (ii).

This theorem was proved by V. Sarkisov in [Sar81]. It has a higherdimensional generalization [Sar82, Theorem 4.1]. Another proof can be found in [Puk00b], see also [SC11, Corollary 7.3] for more general approach.

12.3. Remark. Note that the condition $\left|4 K_{S}+\Delta\right| \neq \varnothing$ follows from the condition that the 1-cycle $-\left(K_{X}\right)^{2}$ is effective.

12.4. Corollary. In the notation and assumptions of Theorem 12.2 the variety $X$ is not birationally equivalent to a $\mathbb{Q}$-Fano threefold and $X$ is not birational to a variety with a structure of $\mathbb{Q}$-del Pezzo fibration. In particular, $X$ is not rational.

Given rationally connected variety $X$, it is an interesting question to describe all birational structures of fibrations on $X$ into varieties with trivial canonical divisor. This is an old classical problem raised by M. Halphen Hal82, Dol66]. For conic bundles, the following necessary condition was obtained by I. Cheltsov:

12.5. Theorem ([Che04]). Let $\pi: X \rightarrow S$ be a standard conic bundle (of any dimension) and let $\Delta$ be the discriminant divisor. If $4 K_{S}+\Delta$ is big, then $X$ is not birationally equivalent to a fibration whose general fiber is a smooth variety with numerically trivial canonical divisor. In particular, $X$ has no birational structure of an elliptic fibration.

This fact can be proved in the same style as 12.2. One has to modify the Noether-Fano inequalities for more general situation.

\section{Birational transformations of $\mathbb{Q}$-CONiC Bundles, II}

In this section we study the transformation $X / S \rightarrow-\rightarrow \bar{X} / \bar{S}$ obtained in Theorem 12.1 in details. We also discuss various applications to rationality problems.

From Theorem 12.1 and the general structure of the Sarkisov program one immediately obtains the following.

13.1. Corollary. In the notation of Theorem 12.1 there exists a link $\bar{\Phi}: \bar{X} / \bar{S} \rightarrow X^{b} / S^{b}$ of one of the following types: 
(i) Type III over a point. Then $X^{b}$ is a $\mathbb{Q}$-Fano threefold with $\rho\left(X^{b}\right)=1$.

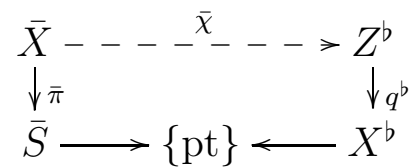

(ii) Type IV over a point. Then $\pi^{b}: X^{b} \rightarrow S^{b}$ is either $a \mathbb{Q}$-conic bundle with $\rho\left(S^{b}\right)=1$ or $S^{b} \simeq \mathbb{P}^{1}$ and $\pi^{b}$ is a $\mathbb{Q}$-del Pezzo fibration.

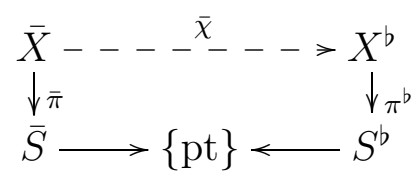

(iii) Type III over a curve. Then $\pi^{b}: X^{b} \rightarrow \mathbb{P}^{1}$ is a $\mathbb{Q}$-del Pezzo fibration.

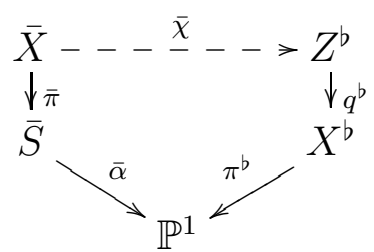

(iv) Type IV over a curve. Then $\pi^{b}: X^{b} \rightarrow S^{b}$ is a $\mathbb{Q}$-conic bundle.

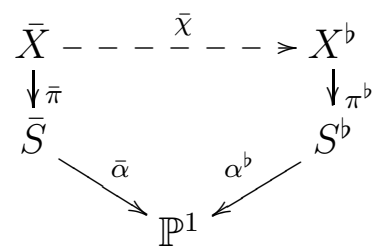

Moreover the link $\bar{\Phi}: \bar{X} / \bar{S} \rightarrow X^{b} / S^{b}$ decreases the coefficient $\mu$ (see (4.3.2)), that is,

$$
\mu^{\mathrm{b}}<\mu,
$$

where $\mu^{b}$ is defined by

$$
\mathscr{H}_{X^{b}} \equiv-\mu^{b} K_{X^{b}}+\pi^{b *} A^{b} .
$$

13.2. Assume that we are in the situation of (13.1.1) or (13.1.2). Then, in particular, $-K_{\bar{S}}$ is ample, i.e. $\bar{S}$ is a del Pezzo surface with at worst $\mathrm{Du}$ Val singularities. All such surfaces have been classified (see, for example, [MZ88, [Dol12, Ch. 8]). In our case, we have additional restrictions: $\rho(\bar{S})=1$ and the singularities of $\bar{S}$ are of type A. According to the classification, there are 28 combinatorial types of Du Val del Pezzo surfaces of Picard number one and among them there are 
exactly 16 types with at worst type A singularities. We reproduce here the complete list, where the notation $\mathrm{nA}_{\mathrm{m}}$ specifies $n$ is the number of $\mathrm{A}_{\mathrm{m}}$ points:

\begin{tabular}{l|l}
$K_{S}^{2}$ & \multicolumn{1}{|c}{$\operatorname{Sing}(S)$} \\
\hline 1 & $4 \mathrm{~A}_{2}, 2 \mathrm{~A}_{1}+2 \mathrm{~A}_{3}, 2 \mathrm{~A}_{4}, \mathrm{~A}_{1}+\mathrm{A}_{2}+\mathrm{A}_{5}, \mathrm{~A}_{1}+\mathrm{A}_{7}, \mathrm{~A}_{8}$ \\
2 & $\mathrm{~A}_{1}+2 \mathrm{~A}_{3}, \mathrm{~A}_{2}+\mathrm{A}_{5}, \mathrm{~A}_{7}$ \\
3 & $3 \mathrm{~A}_{2}, \mathrm{~A}_{1}+\mathrm{A}_{5}$ \\
4 & $2 \mathrm{~A}_{1}+\mathrm{A}_{3}$ \\
5 & $\mathrm{~A}_{4}$ \\
6 & $\mathrm{~A}_{1}+\mathrm{A}_{2}(S \simeq \mathbb{P}(1,2,3))$ \\
8 & $\mathrm{~A}_{1}(S \simeq \mathbb{P}(1,1,2))$ \\
9 & $\varnothing\left(S \simeq \mathbb{P}^{2}\right)$
\end{tabular}

By Theorem 12.1](i) the divisor $-\left(4 K_{\bar{S}}+\bar{\Delta}\right)$ is ample. Clearly, there is only a restricted number of possibilities for $\bar{\Delta}$. Note however that $\bar{\Delta}$ is not necessarily a Cartier divisor on $\bar{S}$ (see Corollary 10.7).

By the adjunction formula we have the following.

13.2.1. Lemma. Let $\bar{S}$ be a del Pezzo surface with at worst Du Val singularities and $\rho(\bar{S})=1$. Let $d:=K_{\bar{S}}^{2}$ and let $\bar{\Delta} \subset \bar{S}$ be a reduced curve. Let $J$ be the ample generator of $\mathrm{Cl}(\bar{S}) /$ tors $\simeq \mathbb{Z}$. Write $-K_{\bar{S}} \equiv$ $\iota J$ and $\bar{\Delta} \equiv a J$ for some positive integers $\iota$ and $a$. Then one of the following holds:

\begin{tabular}{llll}
$d$ & $\bar{S}$ & $\iota$ & $\mathrm{p}_{\mathrm{a}}(\bar{\Delta})$ \\
\hline 9 & $\mathbb{P}^{2}$ & 3 & $(a-3) a / 2+1$ \\
8 & $\mathbb{P}(1,1,2)$ & 4 & $\leq(a-4) a / 4+1$ \\
$\leq 6$ & & $d$ & $\leq(a-d) a / 2 d+1$
\end{tabular}

13.2.2. Corollary. In the above notation, assume that $-\left(4 K_{\bar{S}}+\bar{\Delta}\right)$ is ample, then $a<4 \iota$ and $\mathrm{p}_{\mathrm{a}}(\bar{\Delta}) \leq 45$.

13.2.3. Remark. In some cases the inequalities above can be significantly improved. For example, if in the above notation the divisor $-\left(4 K_{\bar{S}}+\bar{\Delta}\right)$ is ample and $K_{\bar{S}}^{2}=5$, then by Corollary [10.7 the divisor $\bar{\Delta}$ on $\bar{S}$ is Cartier, $a \leq 15$ and $\mathrm{p}_{\mathrm{a}}(\bar{\Delta}) \leq 16$.

13.3. Now assume that we are in the situation of (13.1.4) or (13.1.3). Then there exists a $K_{\bar{S}}$-negative extremal contraction $\bar{\alpha}: \bar{S} \rightarrow \mathbb{P}^{1}:=$ $T$. It is easy to describe the degenerate fibers of this fibration. We leave the following statement as an exercise.

13.3.1. Lemma. Let $(F, C)$ be the germ of a surface with Du Val singularities of type $\mathrm{A}$ along an irreducible reduced curve $C$ such that there exists a $K_{F}$-negative contraction $\delta:(F, C) \rightarrow(T, o)$ to a curve. Then 
$C \simeq \mathbb{P}^{1}$ and for the dual graph of the minimal resolution of $(F, C)$ there are only two possibilities:

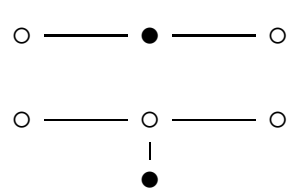

where the vertices $\circ$ correspond to exceptional $(-2)$-curves and $\bullet$ corresponds to $C$. In particular, the singular locus of $F$ consist of two points of type $\mathrm{A}_{1}$ in the case (13.3.2) and one point of type $\mathrm{A}_{3}$ in the case (13.3.3).

13.3.4. Lemma. Let $\varphi: Y \rightarrow Z$ be a $\mathbb{Q}$-conic bundle over a projective rational surface and let $\mathscr{M}$ be a linear system on $Y$ without fixed components. Write $\mathscr{M}+\lambda K_{Y} \equiv \varphi^{*} B$, where $B$ is a $\mathbb{Q}$-divisor on $Z$ and $\lambda \in \mathbb{Q}$. Assume that there exists a base point free pencil $\mathscr{L}$ on $Z$ such that $B \cdot \mathscr{L}<0$. Let $C \subset Y$ be an irreducible curve such that $K_{Y} \cdot C \geq 0$. Then one of the following holds:

(i) $C \subset$ Bs $\mathscr{M}$,

(ii) $\varphi(C) \cdot \mathscr{L}>0$, or

(iii) $\varphi(C)$ is a component of a degenerate fiber of $\mathscr{L}$.

Proof. Clearly, $C$ is not contained in the fibers of $\varphi$. We have

$$
0 \leq \lambda K_{Y} \cdot C=-\mathscr{M} \cdot C+\varphi^{*} B \cdot C .
$$

If $C \not \subset$ Bs $\mathscr{M}$, then $\mathscr{M} \cdot C \geq 0$ and so $\varphi^{*} B \cdot C>0$. In this case, by the projection formula $B \cdot \varphi(C)>0$. Hence, the curve $\varphi(C)$ cannot coincide with a full fiber of the pencil $\mathscr{L}$.

13.3.5. Corollary. In the above notation, let $Y_{\eta}$ be the generic fiber of the composition

$$
Y \stackrel{\varphi}{\longrightarrow} Z \longrightarrow \mathbb{P}^{1}
$$

Then $-K_{Y_{\eta}}$ is ample.

Let $\overline{\mathscr{L}}$ be the base point free pencil of rational curves generated by the fibers of $\bar{\alpha}: \bar{S} \rightarrow \mathbb{P}^{1}$. Let $\bar{X}_{\eta}$ and $\bar{S}_{\eta}$ be generic fibers over $T$ of $\bar{\alpha} \circ \bar{\pi}: \bar{X} \rightarrow T$ and $\bar{\alpha}: \bar{S} \rightarrow T$, respectively. Then $\bar{X}_{\eta}$ is a smooth surface over the non-closed field $\mathbb{k}(T)$ and $\bar{S}_{\eta} \simeq \mathbb{P}_{\mathrm{kk}(T)}^{1}$ is a smooth rational curve. The contraction $\bar{\pi}$ induces a conic bundle structure $\bar{\pi}_{\eta}: \bar{X}_{\eta} \rightarrow \bar{S}_{\eta}$ with

$$
\operatorname{Pic}\left(\bar{X}_{\eta}\right)=\mathbb{Z} \cdot \bar{\Lambda}_{\eta} \oplus \mathbb{Z} \cdot K_{\bar{X}_{\eta}},
$$

where $\bar{\Lambda}_{\eta}$ is the class of the fiber of $\bar{\pi}_{\eta}$. By Corollary 13.3.5 $\bar{X}_{\eta}$ is a del Pezzo surface with $\rho\left(\bar{X}_{\eta}\right)=2$. Since $\log$ flips $\bar{\chi}$ do not change the generic fiber, we also have the following. 
13.3.6. Corollary. The Sarkisov link $\bar{\Phi}: \bar{X} / \bar{S} \rightarrow X^{b} / S^{b}$ induces a link on $\bar{X}_{\eta}$ which is of type $\mathbf{I V}$ in the case (13.1.4) and of type III in the case (13.1.3).

13.3.7. Using the classification of two-dimensional Sarkisov links in Theorems 5.1 and 5.3 we obtain the following possibilities:

(i) $K_{\bar{X}_{\eta}}^{2}=1, \overline{\mathscr{L}} \cdot \bar{\Delta}=7, \pi^{\text {b }}$ is a $\mathbb{Q}$-conic bundle, the link is of type IV and is induced by the Bertini involution $\beta_{\eta}: \bar{X}_{\eta} \rightarrow$ $\bar{X}_{\eta}$. Hence in (13.1.4) we have $\pi^{b}=\lambda \circ \bar{\pi} \circ \beta$ for some birational maps $\beta: \bar{X} \rightarrow \bar{X}$ and $\lambda: S^{b} \rightarrow S^{b}$.

(ii) $K_{\bar{X}_{\eta}}^{2}=2, \overline{\mathscr{L}} \cdot \bar{\Delta}=6, \pi^{b}$ is a $\mathbb{Q}$-conic bundle, the link is of type $\mathbf{I V}$ and is induced by the Geiser involution $\gamma_{\eta}: \bar{X}_{\eta} \rightarrow \bar{X}_{\eta}$. As above, $\pi^{b}=\lambda \circ \bar{\pi} \circ \gamma$ for some birational maps $\gamma: \bar{X} \rightarrow \bar{X}$ and $\lambda: S^{b} \rightarrow S^{b}$.

(iii) $K_{\bar{X}_{\eta}}^{2}=3, \overline{\mathscr{L}} \cdot \bar{\Delta}=5$, the link is of type III and is induced by a contraction of a $(-1)$-curve on $\bar{X}_{\eta} . \pi^{b}: X^{b} \rightarrow \mathbb{P}^{1}$ is a $\mathbb{Q}$-del Pezzo fibration of degree 4.

(iv) $K_{\bar{X}_{\eta}}^{2}=4, \overline{\mathscr{L}} \cdot \bar{\Delta}=4, \pi^{\mathrm{b}}$ is a $\mathbb{Q}$-conic bundle, the link is of type IV. In general, $\bar{\Phi}$ is not induced by a birational self-map of $\bar{X}$.

(v) $K_{\bar{X}_{\eta}}^{2}=5, \overline{\mathscr{L}} \cdot \bar{\Delta}=3$, the link is of type III and is induced by a contraction of four conjugate (-1)-curves on $\bar{X}_{\eta} . \pi^{b}: X^{b} \rightarrow \mathbb{P}^{1}$ is a generically $\mathbb{P}^{2}$-bundle.

(vi) $K_{\bar{X}_{\eta}}^{2}=6, \overline{\mathscr{L}} \cdot \bar{\Delta}=2$, the link is of type III and is induced by a contraction of a pair of conjugate $(-1)$-curves on $\bar{X}_{\eta}$. $\pi^{b}: X^{b} \rightarrow \mathbb{P}^{1}$ is a generically quadric bundle.

13.3.8. Remark. In the cases 13.3.7 (i) and (ii) we obtain a new conic bundle $\pi^{b}: X^{b} \rightarrow S^{b}$ which is fiberwise birational to the original one. On the other hand, by (13.1.5) we have $\lambda^{b}<\lambda$. In order to prove the hard part of Conjecture 1.3, we may assume that the map $X \rightarrow \mathbb{P}^{3}$ is chosen so that $\mu$ is minimal and then these cases 13.3.7](i) (ii) do not occur.

13.3.9. Remark. In the cases 13.3.7 (i), (ii), and(iv), by the construction, for the pencil $\mathscr{L}^{b}$ of fibers of $\alpha^{b}: S^{b} \rightarrow \mathbb{P}^{1}$ and the discriminant curve $\Delta^{b} \subset S^{b}$ we have $\mathscr{L}^{b} \cdot \Delta^{b}=\overline{\mathscr{L}} \cdot \bar{\Delta}$ (see Corollary 13.3.6).

Now we consider an easy case of Conjecture 1.3. More precisely, we assume that in the notation of the proof of Proposition 12.1 the constant $\mu$ equals 1 . Note that in these notation $\mu \in \frac{1}{2} \mathbb{Z}, \mu>0$ and $\mu=1 / 2$ implies that $\Delta=\varnothing$ and $\pi: X \rightarrow S$ is a $\mathbb{P}^{1}$-bundle. 
13.4. Proposition ([Isk87]). Let $\pi: X \rightarrow S$ be a standard conic bundle with discriminant curve $\Delta \subset S$. Assume that there exists a birational map $\Phi: X \rightarrow \mathbb{P}^{3}$ such that for the proper transform $\mathscr{H}$ on $X$ of the linear system of planes on $\mathbb{P}^{3}$ one has $\mathscr{H} \equiv-K_{X}+\pi^{*} A$. Then, for $\pi: X \rightarrow S$, either the condition (i) or the condition (ii) of Conjecture 1.3 is satisfied.

This fact was proved by Iskovskikh in [Isk87, Theorem 2]. We propose below a slightly different proof.

Proof. Clearly, we may assume that $\Delta \neq \varnothing$. Apply Theorem 12.1. Thus we have the diagram (12.1.2) and one of that in Corollary 13.1 with all the required properties. Thus there is a $\mathbb{Q}$-conic bundle $\bar{\pi}$ : $\bar{X} \rightarrow \bar{S}$ that is fiberwise birational to $\pi$ and satisfies the properties in Theorem 12.1. In particular, for the proper transform $\overline{\mathscr{H}}$ of the linear system $\mathscr{H}$ on $\bar{X}$ we have

$$
\overline{\mathscr{H}} \equiv-K_{\bar{X}}+\bar{\pi}^{*} \bar{A}
$$

and the pair $(\bar{X}, \overline{\mathscr{H}})$ is canonical (see the proof of Theorem 12.1).

Assume that we are in the situation(ii) of Theorem 12.1, i.e. $\rho(\bar{S})=$ 2. Thus one of the possibilities 13.3.7(i) (vi) occurs. In the cases 13.3.7) (ii) (ii) and (iv) the fibration $\pi^{b}$ must be a $\mathbb{Q}$-conic bundle with non-trivial discriminant curve by Remark 13.3.9. Since $\mu^{b}<\mu=1$, these cases do not occur. In the case 13.3.7](iii) the fibration $\pi^{b}$ must be a $\mathbb{Q}$-del Pezzo fibration of degree 4 with $\mu^{b}<\mu=1$. Again this is impossible. In the cases 13.3.7) (v) -(vi) the proper transform of $|\overline{\mathscr{L}}|$ on a good model is a desired pencil as in Conjecture 1.3 (i).

Assume now that we are in the case (i) of Theorem 12.1, i.e. $\rho(\bar{S})=$ 1. By (12.1.8) we have

$$
4\left(-K_{\bar{S}}+\bar{A}\right) \equiv \bar{\Delta}+\Gamma,
$$

where $\Gamma=\bar{\pi}_{*}\left(\mathscr{H}_{\bar{X}}\right)^{2}$ is an effective non-zero divisor on $\bar{S}$, and $\bar{A}$ is Cartier by Lemma 10.11. Since $\rho(\bar{S})=1$, by the Noether-Fano inequality (Theorem 4.4) the divisor $-\bar{A}$ is ample. If $\bar{S} \not \mathbb{P}^{2}, \mathbb{P}(1,1,2)$, then $\operatorname{Pic}(\bar{S})=\mathbb{Z} \cdot K_{\bar{S}}$ and we get a contradiction.

Consider the case $\bar{S} \simeq \mathbb{P}(1,1,2)$. Then by (13.4.2) the only possibility is $\bar{A} \equiv \frac{1}{2} K_{\bar{S}}$ and $\bar{\Delta}<-2 K_{\bar{S}}$. Consider the minimal resolution $\mathbb{F}_{2} \rightarrow \bar{S}$. According to Lemma 11.9 there exists a $\mathbb{Q}$-conic bundle $\pi^{\prime}: X^{\prime} \rightarrow \mathbb{F}_{2}$ which completes the map $\mathbb{F}_{2} \rightarrow \bar{S}$ to a Sarkisov link of type I. Let $\Delta^{\prime} \subset \mathbb{F}_{2}$ be the discriminant curve. Write $\Delta^{\prime} \sim a \Sigma+b \Lambda$, where $\Sigma$ and $\Lambda$ are negative section and a fiber of the ruling, respectively. Since $\bar{\Delta}<-2 K_{\bar{S}}$, we have $b \leq 7$. We may assume that $a \geq 4$ (otherwise $|\Lambda|$ gives us a pencil desired in Conjecture 1.3)(i)). But 
then $\Sigma$ is a rational component of $\Delta^{\prime}$ and $\left(\Delta^{\prime}-\Sigma\right) \cdot \Sigma \leq 1$. This is impossible for a discriminant curve (see (3.8.2) and 3.9.1).

Finally, consider the case $\bar{S} \simeq \mathbb{P}^{2}$. We may assume that $\operatorname{deg} \bar{\Delta} \geq 5$. Again by (13.4.2) we have $\operatorname{deg} \bar{\Delta}+\operatorname{deg} \Gamma=8$ and $-\bar{A}$ is the class on a line $l \subset \mathbb{P}^{2}$. Let $\bar{H} \in \mathscr{H}_{\bar{X}}$ and $\bar{M} \in\left|\bar{\pi}^{*} l\right|$ be general members of the corresponding linear systems. Thus, by (13.4.1),

$$
-K_{\bar{X}} \sim \bar{H}+\bar{M} \text {. }
$$

By the adjunction formula the divisor $-K_{\bar{M}}=\left.\bar{H}\right|_{\bar{M}}$ is ample, i.e. $\bar{M}$ is a (smooth) del Pezzo surface. Since the restriction $\left.\mathscr{H}_{\bar{X}}\right|_{\bar{M}}$ defines a birational map to a surface in $\mathbb{P}^{3}$, we have $K_{\bar{M}}^{2} \geq 3$. Using the projection formula and (13.4.2) one obtains

$$
3 \leq K_{\bar{M}}^{2}=\bar{H}^{2} \cdot \bar{M}=\operatorname{deg} \Gamma, \quad \operatorname{deg} \bar{\Delta} \leq 5 .
$$

By our assumption $\operatorname{deg} \bar{\Delta}=5$ and then by the Noether formula $K_{\bar{M}}^{2}=$ 3 .

Suppose that $\bar{\pi}$ is not a standard conic bundle. Thus $\bar{X}$ is singular at some point of a fiber $\bar{\pi}^{-1}(o), o \in \bar{S}$. Let $\pi^{\bullet}: X^{\bullet} \rightarrow S^{\bullet}$ be a standard model of $\bar{\pi}: \bar{X} \rightarrow \bar{S}$ as in Theorem 3.12 and let $\Delta^{\bullet}$ be the corresponding discriminant curve. Thus we have a birational morphism $\alpha: S^{\bullet} \rightarrow \bar{S}$ so that

$$
\Delta^{\bullet} \leq\left(\alpha^{*} \bar{\Delta}\right)_{\text {red }} \quad \text { and } \quad \alpha\left(\Delta^{\bullet}\right)=\bar{\Delta}
$$

(see Corollary 3.3.6). Let $\overline{\mathscr{L}}$ be the pencil of lines on $\bar{S}=\mathbb{P}^{2}$ passing through $o$ and let $\mathscr{L}^{\bullet}$ be its proper transform on $S^{\bullet}$. By the projection formula

$$
\mathscr{L}^{\bullet} \cdot \alpha^{*} \bar{\Delta}=\mathscr{L} \cdot \bar{\Delta}=5 .
$$

By Corollary 10.8 the point $o$ is singular on $\bar{\Delta}$. Let $m_{o}$ the multiplicity of $\bar{\Delta}$ at $o$. Then a general member of $\mathscr{L}^{\bullet}$ meets some component of $\alpha^{*} \bar{\Delta}$ whose coefficient equals $m_{o}$. Hence,

$$
\mathscr{L}^{\bullet} \cdot \Delta^{\bullet} \leq \mathscr{L}^{\bullet} \cdot \alpha^{*} \bar{\Delta}-\left(m_{o}-1\right) \leq 6-m_{o} .
$$

If $m_{o} \geq 3$, then $\mathscr{L}^{\bullet} \cdot \Delta^{\bullet} \leq 3$ and so the condition (i) of Conjecture 1.3 is satisfied. Thus we may assume that $m_{o}=2$. Then by Lemma 10.10 the curves $\alpha^{-1}(o)$ and $\Delta^{\bullet}$ have no common components. Again

$$
\mathscr{L}^{\bullet} \cdot \Delta^{\bullet} \leq \mathscr{L}^{\bullet} \cdot \alpha^{*} \bar{\Delta}-m_{o} \leq 3
$$

and the condition (i) of Conjecture 1.3 is satisfied. Thus we may assume that $\bar{\pi}$ is a standard conic bundle. If the corresponding double cover of $\bar{\Delta}$ is defined by an odd theta-characteristic, then $X$ is not rational by Theorems 7.5 and 7.6. AÓcording to Proposition 8.1 we have the case (ii) of 1.3 . 
Proposition 13.4 shows that Conjecture 1.3 is equivalent to the following classical conjecture of S. Kantor. Recall that a congruence of curves in $\mathbb{P}^{3}$ is an irreducible two-dimensional family of curves that cover $\mathbb{P}^{3}$. The index of a congruence is the number of curves passing through a general point.

13.5. Conjecture (S. Kantor, cf. Kan01, Mil60]). For any congruence of index 1 of rational curves $\mathcal{C}$ in $\mathbb{P}^{3}$ there exists a Cremona transformation $\tau: \mathbb{P}^{3} \rightarrow \mathbb{P}^{3}$ that sends $\mathcal{C}$ to a two-dimensional family of conics or lines.

In other words, Kantor's conjecture can be formulated as follows.

13.5.1. Conjecture. Let $\pi: X \rightarrow S$ be a standard conic bundle. Suppose that there exists a birational map $\Phi: X \rightarrow \mathbb{P}^{3}$. Then there exists a Cremona transformation $\tau: \mathbb{P}^{3} \rightarrow \mathbb{P}^{3}$ such that the composition $\tau \circ \Phi: X \rightarrow \mathbb{P}^{3}$ sends a general fiber either to a conic or to a line.

13.6. Proposition. Conjectures 1.3 and 13.5 are equivalent.

Proof. Let $\pi: X \rightarrow S$ be a standard conic bundle and let $\Phi: X \rightarrow \mathbb{P}^{3}$ be a birational map.

Assume that Conjecture 13.5 holds true. By 13.5.1 we may replace $\Phi$ with another birational map $\Phi^{\prime}=\tau \circ \Phi$ which sends a general fiber either to a conic or to a line. This means that $\mu \leq 1$ (in the notation of the proof of Proposition 12.1). Then by Proposition 13.4 either the condition (i) or the condition (ii) of Conjecture 1.3 is satisfied.

Conversely, assume that Conjecture 1.3 is true. If we are in the situation of 1.3[(ii), then by Proposition 8.1 and Example 3.4 .3 modulo Cremona transformations we have $\mu=1$. In the case 1.3] (i) by Theorem 5.3 we again can see that the images of the fibers of $X^{\prime} / S^{\prime}$ are conics.

13.7. Theorem ([Isk96b]). Let $\pi: X \rightarrow S$ be a standard conic bundle with discriminant curve $\Delta \subset S$. Assume that $\Delta$ is irreducible and smooth. If $X$ is rational, then one of the following holds

(i) $\mathrm{p}_{\mathrm{a}}(\Delta) \leq 45$,

(ii) the condition (i) of Conjecture 1.3 is satisfied,

(iii) $\Delta$ is hyperelliptic and $\pi: X \rightarrow S$ is fiberwise birationally equivalent to a standard conic bundle $\pi^{\bullet}: X^{\bullet} \rightarrow S^{\bullet}$ with discriminant curve $\Delta^{\bullet} \simeq \Delta$ such that there exists a base point free pencil $\mathscr{L}^{\bullet}$ of rational curves on $S^{\bullet}$ such that $\Delta^{\bullet} \cdot \mathscr{L}^{\bullet}=4$.

Iskovskikh Isk96b proposed a stronger assertion. However, the work Isk96b contains serious errors and gaps in the proofs which the author of the review could not eliminate. 
Proof. If $\Delta=0$, then $\pi: X \rightarrow S$ is a locally trivial $\mathbb{P}^{1}$-bundle and there is nothing to prove. Assume now that $\Delta \neq \varnothing$ and $\Phi: X \rightarrow \mathbb{P}^{3}$ is a birational map

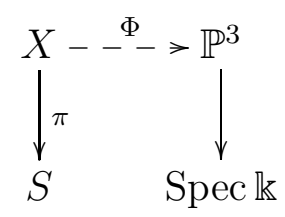

Let $\mathscr{H}_{X} \equiv-\mu K_{X}+\pi^{*} A$ be the proper transform on $X$ of the linear system of planes on $\mathbb{P}^{3}$. Since $\Delta \neq \varnothing$, we have $\mu \in \mathbb{Z}, \mu \geq 1$ and (13.7.1) cannot be completed to a commutative diagram that induces an isomorphism of fibers over generic points, Hence, the hypotheses of Theorem 12.1 are satisfied. Hence, we have the diagram (12.1.2) and one of that in Corollary [13.1 with all the required properties.

The case $\mu=1$ is a consequence of Proposition 13.4.

Assume now that the map $\Phi$ chosen in such a way that $\mu$ has the least possible value. We can thus formulate the following inductive hypothesis:

13.7.2. Conjecture 1.3 holds for all standard conic bundles over a rational surface for which there is a birational map onto $\mathbb{P}^{3}$ with $\mu^{\prime}<\mu$.

We can make use of this hypothesis if we succeed in finding a birational self-map of $X$ that transfers $\pi$ to another birational conic bundle structure on $X$ for which the map $\Phi$ has degree $\mu^{\prime}<\mu$.

Assume that we are in the situation of Theorem 12.1](ii)] Let $\pi^{\bullet}: X^{\bullet} \rightarrow S^{\bullet}$ be a standard conic bundle fiberwise birational to $\bar{\pi}: \bar{X} \rightarrow \bar{S}$ such that $S^{\bullet}$ dominates $\bar{S}$ (see Theorem 3.12). Let $\Delta^{\bullet}$ be the corresponding discriminant curve and let $\left|L^{\bullet}\right|$ be the preimage of the pencil $\overline{\mathscr{L}}$ on $S^{\bullet}$. Then $L^{\bullet} \cdot \Delta^{\bullet}=\overline{\mathscr{L}} \cdot \bar{\Delta}$. Consider the possibilities of 13.3 .7 case by case.

In the cases 13.3.7) and 13.3.7) (vi) we have $L^{\bullet} \cdot \Delta^{\bullet} \leq 3$, so (i) of Conjecture 1.3 is true, as stated in the theorem.

In the cases 13.3.7](i) and 13.3.7)(ii) the $\mathbb{Q}$-conic bundle $\pi^{b}: X^{b} \rightarrow S^{b}$ is fiberwise birational to $\bar{\pi}: \bar{X} \rightarrow S$ and for the corresponding map $X^{b} \rightarrow \mathbb{P}^{3}$ one has $\mu^{b}<\mu$ (see (13.1.5)). Hence by our hypothesis 13.7.2 Conjecture 1.3 is true for $\pi$.

It remains to consider cases 13.3.7](iii) (iv), The curve $\Delta^{\bullet}$ has a component whose normalization is isomorphic to $\Delta$. Since $\Delta^{\bullet}$ satisfies the condition (3.8.2) and $\mathrm{p}_{\mathrm{a}}\left(\Delta^{\bullet}\right)=\mathrm{p}_{\mathrm{a}}(\Delta)$ by (8.4.2), it must be isomorphic to $\Delta$. In the case 13.3.7](iii) (resp. the case 13.3.7](iv)] there is on $\bar{\Delta}$ and thus also on $\Delta^{\bullet}$ a linear series of degree 5 (resp. 4): $q: \Delta^{\bullet} \rightarrow \mathbb{P}^{1}$. Because of the rationality of $X^{\bullet}$ by Theorem 7.5 , we obtain a morphism $p: \Delta^{\bullet} \rightarrow \mathbb{P}^{1}$ of degree 3 or 2 . We claim that the 
morphism

$$
p \times q: \Delta^{\bullet} \rightarrow \Delta^{\prime} \subset \mathbb{P}^{1} \times \mathbb{P}^{1},
$$

is birational. Indeed, otherwise $q$ passes through $p$ :

$$
q: \Delta^{\bullet} \stackrel{p}{\longrightarrow} \mathbb{P}^{1} \stackrel{r}{\longrightarrow} \mathbb{P}^{1}
$$

and $\operatorname{deg} q=(\operatorname{deg} p) \cdot(\operatorname{deg} r)$. This is possible only if $\operatorname{deg} q=4, \operatorname{deg} p=$ $\operatorname{deg} r=2$. In particular, the curve $\Delta^{\bullet}$ is hyperelliptic. Then we have the case (iii). Thus the curve $\Delta^{\prime} \subset \mathbb{P}^{1} \times \mathbb{P}^{1}$ is of bidegree $(n, m)$ with $n \leq 3$ and $m \leq 5$. Hence

$$
\mathrm{p}_{\mathrm{a}}\left(\Delta^{\bullet}\right) \leq \mathrm{p}_{\mathrm{a}}\left(\Delta^{\prime}\right)=(n-1)(m-1) \leq 8,
$$

i.e. we are in the case (i).

Finally, if we are in the case (i) of Theorem 12.1, then by Corollary 13.2 .2 we have $\mathrm{p}_{\mathrm{a}}(\Delta)=\mathrm{p}_{\mathrm{a}}\left(\Delta^{\bullet}\right) \leq 45$, a contradiction. The proof of Theorem 13.7 is complete.

13.8. Note that the inequality $\mathrm{p}_{\mathrm{a}}(\Delta) \leq 45$ in Theorem 13.7) (i) can be strengthened in many cases. For example, if $K_{\bar{S}}^{2} \leq 3$, then $\mathrm{p}_{\mathrm{a}}(\Delta) \leq 15$ by Corollary 13.2.2.

If $K_{\bar{S}}^{2}=5$, then $\mathrm{p}_{\mathrm{a}}(\Delta) \leq 16$ by Remark 13.2 .3

In the case $K_{\bar{S}}^{2}=9$ we have $\bar{S} \simeq \mathbb{P}^{2}$ and $\operatorname{deg} \bar{\Delta} \leq 11$. The pencil of lines passing through a sufficiently general point $s \in \bar{\Delta} \subset \mathbb{P}^{2}$ cuts out on $\bar{\Delta}$ a one-dimensional linear series of degree $\leq 10$. It determines a map $\bar{q}: \bar{\Delta} \rightarrow \mathbb{P}^{1}$ of degree $\leq 10$. Since $s$ is sufficiently general, this linear series is not composed of a hyperelliptic nor trigonal pencil. The map $\bar{q}: \bar{\Delta} \rightarrow \mathbb{P}^{1}$ can be lifted to a finite map $q^{\bullet}: \Delta^{\bullet} \rightarrow \mathbb{P}^{1}$ whose degree is also $\leq 10$. Hence, there is a birational morphism

$$
p^{\bullet} \times q^{\bullet}: \Delta^{\bullet} \rightarrow \tilde{\Delta} \subset \mathbb{P}^{1} \times \mathbb{P}^{1},
$$

where $\tilde{\Delta}$ is of type $\left(d_{1}, d_{2}\right)$ with $d_{1} \leq 3$ and $d_{2} \leq 10$. The birationality follows from the fact that $q^{\bullet}$ does not factor through a hyperelliptic nor trigonal series on $\Delta^{\bullet}$. Hence,

$$
\mathrm{p}_{\mathrm{a}}\left(\Delta^{\bullet}\right) \leq\left(d_{1}-1\right)\left(d_{2}-1\right) \leq 18 .
$$

\section{Some Related RESUlts AND OPEN PROBlems}

14.1. Birational rigidity. It is interesting to have a good criterion for rigidity in terms of discriminant curve and local invariants similar to (3.10.1). A. Corti [Cor00] suggested that a sufficient condition is $\left|3 K_{S}+\Delta\right| \neq \varnothing$ (i.e. et $(S, \Delta) \geq 3$ ). In particular, a standard conic bundle over $\mathbb{P}^{2}$ should be birationally rigid if the discriminant has degree $\geq 9$. See $[\mathrm{BCZ04}]$ for some supporting examples.

Similar to Theorem [13.7 one can prove the following 
14.1.1. Proposition. Let $\pi: X \rightarrow S$ be a standard conic bundle over a rational surface with discriminant curve $\Delta \subset S$. Assume that $X$ is not birationally rigid. Then one of the following holds

(i) $\left|3 K_{S}+\Delta\right|=\varnothing$,

(ii) $X$ is birational to a $\mathbb{Q}$-conic bundle $\bar{\pi}: \bar{X} \rightarrow \bar{S}$ over a $D u$ Val del Pezzo surface as in 13.2. If $\bar{\Delta} \subset \bar{S}$ is the discriminant curve, then $-\left(4 K_{\bar{S}}+\bar{\Delta}\right)$ is ample.

14.2. Unirationality of conic bundles. The unirationality is the most complicated and delicate property of algebraic varieties. Of course one can show that a variety is unirational by explicit geometric construction. However, at the moment there is no techniques to prove non-unirationality (in a non-trivial situation).

First we discuss the unirationality of surfaces over a non-closed field $\mathbb{k}$. A necessary condition for $\mathbb{k}$-unirationality is the existence of a $\mathbb{k}$ point [KSC04, Ex. 1.12]. Any rational surface with a $\mathbb{k}$-point and with $K_{X}^{2} \geq 5$ is $\mathbb{k}$-rational (see Theorem [5.5).

14.2.1. Observation. Let $\pi: X \rightarrow S$ be a rational curve fibration. Then $X$ is $\mathbb{k}$-unirational if and only if $\pi$ has a rational multi-section.

Indeed, if $\sigma: S \rightarrow X$ is a rational multi-section and $C:=\sigma(S)$, then the base change $X \times{ }_{S} C \rightarrow C$ is a rational curve fibration admitting a section. Therefore, $X \times{ }_{S} C$ is $\mathbb{k}$-rational and so $X$ is $\mathbb{k}$ unirational. The converse is easy but there are some difficulties over finite fields (cf. [Kol02, Lemma 2.3]).

14.3. Corollary. Let $\pi: X \rightarrow B$ be a standard conic bundle over a curve. If $K_{X}^{2} \geq 3$ and $X$ has a $\mathbb{k}$-point, then the surface $X$ is either $\mathbb{k}$-rational or $\mathbb{k}$-unirational of degree 2 .

Sketch of the proof. If $K_{X}^{2}=3$, then by Theorem 5.3[(i)] on $X$ there exists a $(-1)$-curve defined over $\mathbb{k}$ which is a double section. Hence we can apply 14.2.1.

Let $K_{X}^{2}=4$ and let $P \in X$ be a $\mathbb{k}$-point. Let $\sigma: \tilde{X} \rightarrow X$ be its blowup and let $E$ be the exceptional divisor. The point $P$ cannot lie on a (-2)-curve according to 5.2](iii).In this situation it is easy to show that the divisor $-K_{\tilde{X}}$ is nef and $\left(-K_{\tilde{X}}\right)^{2}=3$. Moreover, the linear system $\left|-K_{\tilde{X}}-E\right|$ is base point free and defines a morphism $\tilde{X} \rightarrow \mathbb{P}^{1}$ whose generic fiber is a rational curve. Here again $E$ is a double section.

14.3.1. Theorem ([KM17, Theorem 7]). Let $\mathbb{k}$ be a field of characteristic $\neq 2$ and let $\pi: X \rightarrow B$ be a conic bundle over a curve with $K_{X}^{2}=1$. Then $X$ is $\mathbb{k}$-unirational. 
The proof is based on the classification of surface conic bundles with $K_{X}^{2}=1$ (see Proposition 5.2). For example, if $-K_{X}$ is not nef then the curve $C$ from 5.2) is a rational double section. If $-K_{X}$ is nef, i.e. $X$ is a weak del Pezzo surface, and the Bertini involution $\beta: X \rightarrow X$ does not preserve the conic bundle structure, then the image $\beta(F)$ of a fiber $F$ is a rational multisection. Thus the only interesting case is the case where $-K_{X}$ is nef and the Bertini involution $\beta: X \rightarrow X$ acts fiberwise (see [KM17]).

14.3.2. Corollary. Let $\mathbb{k}$ be a field of characteristic $\neq 2$ and let $\pi$ : $X \rightarrow B$ be a conic bundle over a curve. Assume that $K_{X}^{2} \geq 1$. Then $X$ is $\mathbb{k}$-unirational if and only if it has a $\mathbb{k}$-point.

On the other hand, Iskovskikh [Isk67] had shown that, in the case $\mathbb{k}=\mathbb{R}$, any rational surface over $\mathbb{k}$ with conic bundle structure is $\mathbb{k}$-unirational whenever it has a $\mathbb{k}$-point. In particular, this implies that there are $\mathbb{k}$-unirational conic bundles whose discriminant locus is arbitrarily large and so we cannot expect that a unirationality criterion can be formulated in the form similar to Theorem 5.5. Unirationality of surface conic bundles over some "large" fields were discussed in [VY86], [Yan85, Yan92.

Unirationality of del Pezzo surfaces of degree $\geq 3$ with a $\mathbb{k}$-point was proved in [Man74], KKol02]. It is expected that a del Pezzo surface of degree 2 with a $\mathbb{k}$-point is always unirational. This was proved in many but not in all cases, see [Man74], [STVA14], [FvL16]. It is very little known about unirationality del Pezzo surfaces of degree 1.

Manin observed that the invariant

$$
H^{1}(\operatorname{Gal}(\overline{\mathbb{k}} / \mathbb{k}), \operatorname{Pic}(X \otimes \overline{\mathbb{k}}))
$$

allows to bound the degree of unirationality [Man74, §IV.7].

As an immediate consequence of 14.2.1, one can see also that a cubic hypersurface in $\mathbb{P}^{4}$ and an intersection of three quadrics in $\mathbb{P}^{6}$ are unirational (because they have structures of conic bundles with rational multisections, see Examples 3.4.2 and 3.4.4). As a consequence of Corollary 14.3.2 we obtain also a sufficient condition of unirationality of three-dimensional conic bundles over $\mathbb{C}$ :

14.3.3. Corollary (cf. Mel14). Let $\pi: X \rightarrow S$ be a standard conic bundle over a surface and let $\Delta \subset S$ be the discriminant curve. Assume that there is a base point free pencil of rational curves $\mathscr{L}$ on $S$ such that $\Delta \cdot \mathscr{L} \leq 7$. Then $X$ is unirational.

14.3.4. Corollary. Let $\pi: X \rightarrow \mathbb{P}^{2}$ be a standard conic bundle and let $\Delta$ be the discriminant curve. If $\operatorname{deg} \Delta \leq 8$, then $X$ is unirational. 
It is likely that a conic bundle with sufficiently large and general discriminant curve is not unirational.

14.4. Stable rationality. In a joint paper BCTSSD85] the authors constructed a counterexample to the birational Zariski cancellation problem.

14.4.1. Theorem ([BCTSSD85]). Let $\mathbb{k}$ be a field of characteristic $\neq 2$, and $p(x) \in \mathbb{k}[x]$ an irreducible separable polynomial of degree 3 with discriminant $a \in \mathbb{k}^{*} \backslash\left(\mathbb{k}^{*}\right)^{2}$. Then the surface $X$ given by the affine equation

$$
y^{2}-a z^{2}=p(x)
$$

has the property that $X \times \mathbb{P}^{3}$ is $\mathbb{k}$-rational but $X$ itself is not.

The stable rationality of the surface (14.4.2) was established by the technique of torsors. The projection to $\mathbb{A}_{x}^{1}$ induces a conic bundle structure on a suitable projective model of $X$. Then the nonrationality of $X$ follows from Theorem 5.5. The result was improved by $\mathrm{N}$. Shepherd-Barron [SB04]: one can replace $X \times \mathbb{P}^{3}$ with $X \times \mathbb{P}^{2}$.

Over $\mathbb{C}$, an example a stable rational non-rational algebraic threefold $X_{0}$ can be given by the affine equation

$$
y^{2}-a(t) z^{2}=p(t, x)
$$

for a suitable polynomial $p(t, x)$ and $a(t)=\operatorname{disc}_{x} p(t, x)$. The variety $X_{0}$ is birationally equivalent to a standard conic bundle $\pi: X \rightarrow S$ over a rational surface. The corresponding discriminant curve $\Delta$ is a union of a trigonal curve $C$ of genus $g$ and smooth rational curves $L, F_{1}, \ldots, F_{2 g+4}$ so that the singularities of $\Delta$ are the double points $\left\{P_{i}\right\}=C \cap F_{i}$, and $\left\{R_{i}\right\}=L \cap F_{i}$. See [BCTSSD85, §3] for details.

On the other hand, it turns out that stably rational non-rational varieties are very rare. In the paper [HKT16] the authors applied specialization technique of Voisin [Voi15], developed by Totaro [Tot16], Colliot-Thélène and Pirutka CP16b], CP16a to conic bundles:

14.4.3. Theorem ([HKT16, see also [BG]). Let $S$ be a smooth projective rational surface. Let $\mathscr{L}$ be a linear system on $S$ whose general member is smooth and irreducible. Let $\mathscr{M}$ be an irreducible component of the space of reduced nodal curves in $\mathscr{L}$ together with degree 2 étale cover. Assume that $\mathscr{M}$ contains a cover which is nontrivial over every irreducible component of a reducible curve with smooth irreducible components. Then the standard conic bundle corresponding to a very general point of $\mathscr{M}$ is not stably rational.

14.4.4. Corollary. A very general conic bundle $\pi: X \rightarrow \mathbb{P}^{2}$ with discriminant curve of degree $\geq 6$ is not stably rational. 
Higher-dimensional analog of this result see in AO16]. The stable rationality has an interesting analogue in the category of $G$-varieties [BP13], Pro15c].

14.5. Birationality to Calabi-Yau pairs. We say that a pair $(Y, D)$ consisting of a projective variety $X$ and an effective $\mathbb{R}$-divisor $D$ is a $\log$ Fano variety if it has only Kawamata log terminal singularities and $-\left(K_{X}+D\right)$ is ample. If some variety $X$ admits an effective $\mathbb{R}$ divisor $D$ so that $(Y, D)$ is a $\log$ Fano, we say that $X$ is of Fano type [PS09]. Usual Fano varieties and toric varieties are standard examples of varieties of Fano type. It is known by that any $\mathbb{Q}$-factorial variety of Fano type is a Mori dream space [BCHM10]. It turns out that Fano type varieties satisfy many important properties: they are rationally connected, and this class is closed under the MMP, as well as, under arbitrary contractions PS09.

We say that $(Y, D)$ is a $\log$ Calabi-Yau pair if $K_{X}+D \sim_{\mathbb{Q}} 0$ and $(Y, D)$ is $\log$ canonical, where $D$ is an effective $\mathbb{R}$-divisor. We say that $Y$ is of Calabi-Yau type if $(Y, D)$ is log Calabi-Yau for some $D$. Finally, we say that $(Y, D)$ is a numerical log Calabi-Yau pair if $K_{X}+D \equiv 0$, where $D$ is pseudo-effective (no restrictions on the singularities of $Y$ are imposed). As above, one can define varieties of numerically CalabiYau type. By [MM86], for any numerical log Calabi-Yau pair $(X, D)$ with $D \neq 0$, the underlying variety $Y$ is uniruled.

It is known that log Fano varieties with bounded singularities form a bounded family Bir16a. Thus the following question is natural.

14.5.1. Question. Under what conditions a conic bundle $\pi: X \rightarrow S$ is of Fano type (resp. Calabi-Yau type, numerically Calabi-Yau type)?

The following result of J. Kollár shows that the condition for a variety even to be numerically Calabi-Yau type is very restrictive.

14.5.2. Theorem ([Kol17]). Let $\pi: X \rightarrow \mathbb{P}^{2}$ be a standard conic bundle whose discriminant curve $\Delta \subset \mathbb{P}^{2}$ has degree $\geq 19$. The following are equivalent:

(i) $X$ is birational to a variety of numerically Calabi-Yau type.

(ii) There is a generically finite double section $D \subset X$ with normalization $\tau: \bar{D} \rightarrow D$ such that the branch curve of $\pi \circ \tau: \bar{D} \rightarrow \mathbb{P}^{2}$ has degree $\leq 6$.

(iii) $X$ is birational to a standard conic bundle $\pi^{\prime}: X^{\prime} \rightarrow \mathbb{P}^{2}$ (with the same discriminant curve) such that $\left|-K_{X^{\prime}}\right| \neq 0$.

As a consequence one has. 
14.5.3. Theorem ([Kol17]). Let $X_{d, 2} \subset \mathbb{P}^{2} \times \mathbb{P}^{2}$ be a general hypersurface of bidegree $(d, 2)$. Then $X_{d, 2}$ is not birational to a variety of numerically Calabi-Yau type for $d \geq 7$.

There are analogs of these results for surfaces over non-closed fields. Similar question for del Pezzo fibrations was studied by I. Krylov [Kry18.

14.6. Conic bundle structures on Fano threefolds. The following question is natural:

14.6.1. Question. Which (smooth) Fano threefolds admit a birational conic bundle structure?

Recall [IP99, § 2.1] that the Fano index $\iota=\iota(X)$ of an $n$-dimensional Fano manifold $X$ is the maximal integer such that $-K_{X}=\iota A$, where $A$ is an (integral) divisor. The number $d=\mathrm{d}(X):=A^{n}$ is called the degree of $X$. In the case $\iota(X)=n-2$, the degree is even and so the number $g=\mathrm{g}(X):=\mathrm{d}(X) / 2+1$ is an integer. It is called the genus of $X$.

Let us summarize the known facts about conic bundle structures on Fano threefolds with $\rho(X)=1$. We use the classification and terminology of [IP99, Table $\S 12.2$ ].

Case $\iota>2$. If $\iota=4$, then $X \simeq \mathbb{P}^{3}$; if $\iota=3$, then $X \simeq Q \subset \mathbb{P}^{4}$ is a quadric. These varieties are rational and so they have a lot of birational conic bundle structures.

Case $\iota=2, d \geq 4$. It is known that $d \leq 5$. For $d=5$ and 4 the Fano threefolds are rational.

Case $\iota=2, d=3$. Then $X \simeq X_{3} \subset \mathbb{P}^{4}$ is a cubic threefold. It has a lot of conic bundle structures (see Example 3.4.2).

Case $\iota=2, d=2$. Then $X=X_{2}$ can be represented as double cover $X \rightarrow \mathbb{P}^{3}$ branched over a quartic (it is so-called quartic double solid). The existence of conic bundle structure on smooth varieties of this type in not known. However, if $X$ has at least one ordinary double point, say $P$, then the "projection" from $P$ gives a conic bundle, see Examples 3.4.7 and 6.5.

Rationality questions for singular quartic double solids were studied in [Cle83], [Voi88], [Var86], [Deb90], [CPS15], [Voi15]. 
Case $\iota=2, d=1$. Then $X=X_{1}$ is so-called double Veronese cone. The birational geometry of this variety is very rich. It was studied in the series of papers of M. Grinenko (see [Gri03], Gri04]). In particular, he proved that there is no conic bundle structures on $X_{1}$.

If $\iota=1$, then $g \in\{2,3, \ldots, 10,12\}$.

Case $\iota=1, g \in\{7,9,10,12\}$. Then the variety $X$ is rational.

Case $\iota=1, g=8$. The double projection from a line gives a structure of a conic bundle structure [IP99, Theorem 4.3.3]. Conic bundle structures exist also because $X$ is birational to a cubic threefold [Isk80a], [Tre85], [IP99, Theorem 4.5.8] (see Example 3.4.2).

Case $\iota=1, g=6$. The existence of conic bundle structures is unknown for smooth varieties of this type. However, singular Fano threefolds with $\iota=1, g=6$ may have conic bundle structures. For example, if such a variety $X$ is $\mathbb{Q}$-factorial and has a unique node, then is can be birationally transformed to a standard conic bundle over $\mathbb{P}^{2}$ with discriminant curve a smooth sextic $\Delta \subset \mathbb{P}^{2}$ [DIM11], [Pro17]. If $X$ is not $\mathbb{Q}$-factorial and has a unique node, then it can be transformed to a smooth quartic double solid (see above) by [DIM12, Proposition 5.2], [PCS05, Example 1.11], or [BCZ04, §4.4.1].

Case $\iota=1, g=5$. Then $X \simeq X_{2 \cdot 2 \cdot 2} \subset \mathbb{P}^{6}$ is an intersection of three quadrics. Conic bundle structures exist by Example 3.4.4.

Case $\iota=1, g=4$. Then $X \simeq X_{2 \cdot 3} \subset \mathbb{P}^{5}$ is an intersection of a quadric and a cubic. A general member of the family is birationally rigid and has no conic bundle structures [IP96, Ch. 3, § 2], [Puk13, Ch. 2, §§ 5-6]. However, this is not known for any smooth variety of this type.

Case $\iota=1, g=3$. Then $X$ is either a quartic in $\mathbb{P}^{4}$ or a double cover a quadric branched over a surface of degree 8 ( $X$ is said to be a double quadric). In both cases $X$ is birationally rigid and has no conic bundle structures (see [IM71, [Isk80a]).

Case $\iota=1, g=2$. Then $X$ is a so-called sextic double solid. It is birationally superrigid and so it has no conic bundle structures [Isk80a].

More examples of singular Fano threefolds with birational conic bundle structure can be found in [JPR11, $\S \S 7.2,7.5-7.7$. In contrast with the above considered case $\rho(X)=1$, many Fano threefolds with $\rho(X)>1$ have (even biregular) conic bundle structures [MM82, see also [Kuz15] for higher-dimensional examples. 
Note that Kollár's method of reduction to positive characteristic [Ko195] allows to show that certain Fano hypersurfaces of high dimension do not admit a birational conic bundle structures.

14.7. Del Pezzo fibrations. It is desirable to construct a good theory of three-dimensional del Pezzo fibrations. Some generalization of Sarkisov's theorem on standard models was obtained by A. Corti [Cor96] (see also [Kol97], [Log17]). For del Pezzo fibrations of degree $\geq 3$ he proved that there exist a Gorenstein terminal model such that its fibers are reduced and irreducible. For del Pezzo fibrations of degree 2 and 1 the situation is more complicated: it turns out that a Gorenstein terminal model does not always exist, one should consider models with non-Gorenstein points of low indices.

A del Pezzo fibration of degree $\geq 5$ over $\mathbb{P}^{1}$ is always rational (cf. Theorem 5.5). Any del Pezzo fibration $\pi: X \rightarrow B$ of degree 4 birationally has a conic bundle structure (cf. Theorem 5.3 (i)). In the case of smooth $X$, the rationality criterion was obtained by Alexeev [Ale87] (see also [Shr06b]). The case of del Pezzo fibrations of degree $\leq 3$ is much more complicated. For results on birational rigidity we refer to [Isk95], [Puk98], [Puk13, Ch. 4, § 1], Che05], Sob02], Gri00], [SC11, Kry16. A higher-dimensional generalization was discussed in a recent paper [Puk17]. It would be interesting to reformulate these results in terms of degeneracy loci and local invariants. There are also results on local birational rigidity [Par01], Par03], Puk00a].

14.8. Extremal contractions of relative dimension one. Consider a Mori extremal contraction $\pi: X \rightarrow S$ from a smooth $n$ dimensional variety such that the generic fiber is of dimension 1 . If $n \leq 3$, then $S$ is smooth and $\pi$ is a standard conic bundle Mor82. The same holds in arbitrary dimension under the additional assumption that all the fibers are one-dimensional [And85. However, if we relax the equidimensionality condition, then the situation becomes more complicated even for $n=4$.

14.9. Proposition. Let $\pi: X \rightarrow S$ be a Mori extremal contraction with $0<\operatorname{dim} S<\operatorname{dim} X$. Assume that $X$ is smooth. Then the following holds.

(i) S has at worst locally factorial canonical singularities.

(ii) For any point $P \in S$, its local algebraic fundamental group is trivial.

(iii) $\operatorname{codim} \operatorname{Sing}(S) \geq 3$. In particular, $S$ is smooth if $\operatorname{dim} S \leq 2$.

(iv) (cf. [AW97, Proposition 1.3]) If $S$ has at worst quotient singularities, then it is smooth.

(v) If $\pi$ is equidimensional, then $S$ is smooth. 
Proof. (i) Recall that in general the singularities of $S$ are rational [Kol86, Corollary 7.4]. The factoriality of $S$ is proved in the same style as [KMM87, Lemma 5-1-5] or [KM98, Corollary 3.18]. For convenience of the reader we reproduce these arguments. Let $B$ be a prime Weil divisor on $S$ and let $D$ be the divisorial part of $\pi^{-1}(B)$. Then $D$ is a Cartier divisor on $X$ and $\pi(D)=B$. Since $D$ does not meet a general fiber, $D=\pi^{*} B_{0}$ for some Cartier divisor on $S$ [KM98, Corollary 3.17]. Clearly, $B_{0} \subset B$ and so $B_{0}=B$. Thus, the singularities of $S$ are rational and $K_{S}$ is a Cartier divisor. In this case the singularities must be canonical (see [KM98, Corollary 5.24]).

(ii) Regard $S$ as a sufficiently small neighborhood of $o$. Assume that there is a Galois cover $\left(S^{\prime} \ni o^{\prime}\right) \rightarrow(S \ni o)$ which is étale over $S \backslash \operatorname{Sing}(S)$. Thus $(S \ni o)=\left(S^{\prime} \ni o^{\prime}\right) / G$ and $G$ is a finite group acting on $S^{\prime}$ freely in codimension one. Consider the normalization $X^{\prime}$ of the fiber product of $X \times_{S} S^{\prime}$. Then $f: X^{\prime} \rightarrow X$ is a finite Galois morphism which is étale in codimension one. Thus, by purity of the branch locus, $f$ is étale and $X^{\prime}$ is smooth. The projection $\pi^{\prime}: X^{\prime} \rightarrow S^{\prime}$ is a contraction such that $-K_{X^{\prime}}$ is relatively ample. By [PS16c, Lemma 3.4] there exists an irreducible $G$-invariant rationally connected subvariety $V \subset \pi^{\prime-1}\left(o^{\prime}\right)$. On the other hand, an action of a cyclic subgroup $G_{1} \subset G$ on a rationally connected variety always has a fixed point. Hence, the action of $G$ on $V$ and $X^{\prime}$ cannot be free and so $G=\{1\}$.

Assertions (iii) and (iv) easily follow from (i) and (ii). Finally, for (v), we just have to note that in this case a general section $H$ of $X$ by subspace of codimension $\operatorname{dim} X-\operatorname{dim} S$ is smooth and the restriction $\pi_{H}: H \rightarrow S$ is finite, hence $S$ has at worst quotient singularities.

Kachi [Kac97 classified contractions from smooth fourfold to threefolds. In this case the base variety is not necessarily smooth.

14.10. Equivariant conic bundles. It would be useful to develop the theory of conic bundles in the category of $G$-varieties [Man67], that is, for varieties over non-closed fields, as well as, for varieties with a group action. The first step was done by A. Avilov Avi14. He proved an analog of Theorem 3.12, We hope that Theorems 12.2, 12.5, and Conjectures 1.3 and 1.2 should be generalized for the equivariant case. This is important for applications to the study of Cremona groups and rationality problems, see [PS17], [PS16a], [PS16b], Pop15], [Yas16], [Yas17, [Pop17], [PS16d], [Tre14], [Tre16]. 


\section{REFERENCES}

[AB92] Alberto Alzati and Marina Bertolini. On the rationality of Fano 3folds with $B_{2} \geq 2$. Matematiche, 47(1):63-74, 1992.

[AB14] Valery Alexeev and Alexander Borisov. On the log discrepancies in toric Mori contractions. Proc. Amer. Math. Soc., 142(11):3687-3694, 2014.

[AK77] Allen B. Altman and Steven L. Kleiman. Foundations of the theory of Fano schemes. Compositio Math., 34(1):3-47, 1977.

[Ale87] V. A. Alekseev. On conditions for the rationality of three-folds with a pencil of del Pezzo surfaces of degree 4. Math. Notes, 41(5):408-411, 1987.

[Ale94a] Valery Alexeev. Boundedness and $K^{2}$ for log surfaces. Internat. $J$. Math., 5(6):779-810, 1994.

[Ale94b] Valery Alexeev. General elephants of Q-Fano 3-folds. Compositio Math., 91(1):91-116, 1994.

[AM72] M. Artin and D. Mumford. Some elementary examples of unirational varieties which are not rational. Proc. London Math. Soc. (3), 25:7595, 1972.

[And85] Tetsuya Ando. On extremal rays of the higher-dimensional varieties. Invent. Math., 81(2):347-357, 1985.

[AO16] Hamid Ahmadinezhad and Takuzo Okada. Stable rationality of higher dimensional conic bundles. Arxiv e-print, 1612.04206, 2016.

[Avi14] A. A. Avilov. Existence of standard models of conic fibrations over non-algebraically-closed fields. Sb. Math., 205(12):1683-1695, 2014.

[AW97] Marco Andreatta and Jarosław A. Wiśniewski. A view on contractions of higher dimensional varieties. In Algebraic geometry. Proceedings of the Summer Research Institute, Santa Cruz, CA, USA, July 9-29, 1995, pages 153-183. Providence, RI: American Mathematical Society, 1997.

$\left[\mathrm{B}^{+}\right] \quad$ Gavin Brown et al. Graded Ring Database. http://www.grdb.co.uk.

[BCHM10] Caucher Birkar, Paolo Cascini, Christopher D. Hacon, and James McKernan. Existence of minimal models for varieties of log general type. J. Amer. Math. Soc., 23(2):405-468, 2010.

[BCTSSD85] Arnaud Beauville, Jean-Louis Colliot-Thélène, Jean-Jacques Sansuc, and Peter Swinnerton-Dyer. Variétés stablement rationnelles non rationnelles. Ann. of Math. (2), 121(2):283-318, 1985.

[BCZ04] Gavin Brown, Alessio Corti, and Francesco Zucconi. Birational geometry of 3-fold Mori fibre spaces. In The Fano Conference, pages 235-275. Univ. Torino, Turin, 2004.

[Bea77a] Arnaud Beauville. Prym varieties and Schottky problem. Invent. Math., 41:149-196, 1977.

[Bea77b] Arnaud Beauville. Variétés de Prym et jacobiennes intermédiaires. Ann. Sci. École Norm. Sup. (4), 10(3):309-391, 1977.

[Bea82] Arnaud Beauville. Les singularités du diviseur $\Theta$ de la jacobienne intermédiaire de l'hypersurface cubique dans $\mathbf{P}^{4}$. In Algebraic threefolds (Varenna, 1981), volume 947 of Lecture Notes in Math., pages 190-208. Springer, Berlin, 1982.

[Bea89] Arnaud Beauville. Prym varieties: a survey. In Theta functionsBowdoin 1987, Part 1 (Brunswick, ME, 1987), volume 49 of Proc. 
Sympos. Pure Math., pages 607-620. Amer. Math. Soc., Providence, RI, 1989.

[Bea00] Arnaud Beauville. Determinantal hypersurfaces. Michigan Math. J., 48:39-64, 2000.

[Bea16] Arnaud Beauville. A very general sextic double solid is not stably rational. Bull. Lond. Math. Soc., 48(2):321-324, 2016.

[Bel85] Mauro Beltrametti. On the Chow group and the intermediate Jacobian of a conic bundle. Ann. Mat. Pura Appl. (4), 141:331-351, 1985.

[BG] Christian Böhning and Hans-Christian Graf von Bothmer. On stable rationality of some conic bundles and moduli spaces of Prym curves . Arxiv e-print, 1605.03029.

[Bir16a] Caucher Birkar. Singularities of linear systems and boundedness of Fano varieties. Arxiv e-print, 1609.05543, 2016.

[Bir16b] Caucher Birkar. Singularities on the base of a Fano type fibration. J. Reine Angew. Math., 715:125-142, 2016.

[BL12] Jérémy Blanc and Stéphane Lamy. Weak Fano threefolds obtained by blowing-up a space curve and construction of Sarkisov links. Proc. Lond. Math. Soc. (3), 105(5):1047-1075, 2012.

[Bog88] F.A. Bogomolov. Brauer groups of factor spaces of linear representations. Math. USSR-Izv., 30(3):455-485, 1988.

[BP13] F. Bogomolov and Yu. Prokhorov. On stable conjugacy of finite subgroups of the plane Cremona group, I. Cent. European J. Math., 11(12):2099-2105, 2013.

[CFHR99] Fabrizio Catanese, Marco Franciosi, Klaus Hulek, and Miles Reid. Embeddings of curves and surfaces. Nagoya Math. J., 154:185-220, 1999.

[CG72] C. Herbert Clemens and Phillip A. Griffiths. The intermediate Jacobian of the cubic threefold. Ann. of Math. (2), 95:281-356, 1972.

[Che04] I. A. Cheltsov. Conic bundles with a large discriminant. Izv. Math., 68(2):429-434, 2004.

[Che05] Ivan Cheltsov. Birationally rigid del Pezzo fibrations. Manuscripta Math., 116(4):385-396, 2005.

[Che09] Ivan Cheltsov. Points in projective spaces and applications. J. Differential Geom., 81(3):575-599, 2009.

[Cle75] C.H. Clemens. Applications of the theory of Prym varieties. In Proc. int. Congr. Math., Vancouver 1974, volume 1, pages 415-421, 1975.

[Cle83] C. Herbert Clemens. Double solids. Adv. in Math., 47(2):107-230, 1983.

[CM77a] A. Conte and J. P. Murre. On quartic threefolds with a double line. I. Nederl. Akad. Wetensch. Proc. Ser. A 80=Indag. Math., 39(3):145160, 1977.

[CM77b] A. Conte and J. P. Murre. On quartic threefolds with a double line. II. Nederl. Akad. Wetensch. Proc. Ser. A 80=Indag. Math., 39(3):161$175,1977$.

[Cor95] Alessio Corti. Factoring birational maps of threefolds after Sarkisov. J. Algebraic Geom., 4(2):223-254, 1995.

[Cor96] Alessio Corti. Del Pezzo surfaces over Dedekind schemes. Ann. of Math. (2), 144(3):641-683, 1996. 
[Cor00] Alessio Corti. Singularities of linear systems and 3-fold birational geometry. In Explicit birational geometry of 3-folds, volume 281 of London Math. Soc. Lecture Note Ser., pages 259-312. Cambridge Univ. Press, Cambridge, 2000.

[CP16a] Jean-Louis Colliot-Thélène and Alena Pirutka. Hypersurfaces quartiques de dimension 3: non-rationalité stable. Ann. Sci. Éc. Norm. Supér. (4), 49(2):371-397, 2016.

[CP16b] Jean-Louis Colliot-Thélène and Alena V. Pirutka. Cyclic covers that are not stably rational. Izv. Math., 80(4):665-677, 2016.

[CPS15] Ivan Cheltsov, Victor Przyjalkowski, and Constantin Shramov. Which quartic double solids are rational? Arxiv e-print, 1508.07277, 2015.

[CT88] D.F. Coray and M.A. Tsfasman. Arithmetic on singular del Pezzo surfaces. Proc. Lond. Math. Soc., III. Ser., 57(1):25-87, 1988.

[CTS07] Jean-Louis Colliot-Thélène and Jean-Jacques Sansuc. The rationality problem for fields of invariants under linear algebraic groups (with special regards to the Brauer group). In Algebraic groups and homogeneous spaces, Tata Inst. Fund. Res. Stud. Math., pages 113-186. Tata Inst. Fund. Res., Mumbai, 2007.

[Cut88] Steven Cutkosky. Elementary contractions of Gorenstein threefolds. Math. Ann., 280(3):521-525, 1988.

[Deb89] Olivier Debarre. Sur le problème de Torelli pour les variétés de Prym. Am. J. Math., 111(1):111-124, 1989.

[Deb90] Olivier Debarre. Sur le théorème de Torelli pour les solides doubles quartiques. Compos. Math., 73(2):161-187, 1990.

[DIM11] Olivier Debarre, Atanas Iliev, and Laurent Manivel. On nodal prime Fano threefolds of degree 10. Sci. China Math., 54(8):1591-1609, 2011.

[DIM12] Olivier Debarre, Atanas Iliev, and Laurent Manivel. On the period map for prime Fano threefolds of degree 10. J. Algebraic Geom., 21(1):21-59, 2012.

[Dol66] I. V. Dolgachev. Rational surfaces with a pencil of elliptic curves. Izv. Akad. Nauk SSSR Ser. Mat., 30:1073-1100, 1966.

[Dol12] Igor V. Dolgachev. Classical algebraic geometry. Cambridge University Press, Cambridge, 2012.

[Ehn85] S.Yu. Ehndryushka. Nonrationality of the general Enriques variety. Math. USSR, Sb., 51:267-273, 1985.

[Fuj99] Osamu Fujino. Applications of Kawamata's positivity theorem. Proc. Japan Acad. Ser. A Math. Sci., 75(6):75-79, 1999.

[FvL16] Dino Festi and Ronald van Luijk. Unirationality of del Pezzo surfaces of degree 2 over finite fields. Bull. Lond. Math. Soc., 48(1):135-140, 2016.

[GHS03] Tom Graber, Joe Harris, and Jason Starr. Families of rationally connected varieties. J. Am. Math. Soc., 16(1):57-67, 2003.

[Gri00] M. M. Grinenko. Birational properties of pencils of del Pezzo surfaces of degrees 1 and 2. Mat. Sb., 191(5):17-38, 2000.

[Gri03] M. M. Grinenko. On the double cone over the Veronese surface. Izv. Math., 67(3):421-438, 2003. 
[Gri04] M. M. Grinenko. Mori structures on a Fano threefold of index 2 and degree 1. Proc. Steklov Inst. Math., 246:103-128, 2004.

[Hal82] G. Halphén. Sur les courbes planes du sixième degré à neuf points doubles. Bull. Soc. Math. France, 10:162-172, 1882.

[Har77] Robin Hartshorne. Algebraic geometry. Springer-Verlag, New York, 1977. Graduate Texts in Mathematics, No. 52.

[Hay99] Takayuki Hayakawa. Blowing ups of 3-dimensional terminal singularities. Publ. Res. Inst. Math. Sci., 35(3):515-570, 1999.

[Hay00] Takayuki Hayakawa. Blowing ups of 3-dimensional terminal singularities. II. Publ. Res. Inst. Math. Sci., 36(3):423-456, 2000.

[HKT16] Brendan Hassett, Andrew Kresch, and Yuri Tschinkel. Stable rationality and conic bundles. Math. Ann., 365(3-4):1201-1217, 2016.

[HM13] Christopher D. Hacon and James McKernan. The Sarkisov program. J. Algebr. Geom., 22(2):389-405, 2013.

[HMX14] Christopher D. Hacon, James McKernan, and Chenyang Xu. ACC for log canonical thresholds. Ann. of Math. (2), 180(2):523-571, 2014.

[IKP14] Atanas Iliev, Ludmil Katzarkov, and Victor Przyjalkowski. Double solids, categories and non-rationality. Proc. Edinb. Math. Soc., II. Ser., 57(1):145-173, 2014.

[IM71] V. A. Iskovskikh and Ju. I. Manin. Three-dimensional quartics and counterexamples to the Lüroth problem. Mat. Sb. (N.S.), 86(128):140-166, 1971.

[IP96] V. A. Iskovskikh and A. V. Pukhlikov. Birational automorphisms of multidimensional algebraic manifolds. J. Math. Sci., 82(4):35283613, 1996.

[IP99] V. A. Iskovskikh and Yu. Prokhorov. Fano varieties. Algebraic geometry V., volume 47 of Encyclopaedia Math. Sci. Springer, Berlin, 1999.

[IS05] V. A. Iskovskikh and V. V. Shokurov. Birational models and flips. Russ. Math. Surv., 60(1):27-94, 2005.

[Isk67] V. A. Iskovskikh. Rational surfaces with a pencil of rational curves. Mat. USSR Sbornik, 3(4):563-587, 1967.

[Isk70] V. A. Iskovskikh. Rational surfaces with a pencil of rational curves and with positive square of the canonical class. Mat. Sb., 83(1):90$119,1970$.

[Isk72] V.A. Iskovskikh. Birational properties of a surface of degree 4 in $\mathbb{P}_{k}^{4}$. Math. USSR, Sb., 17:30-36, 1972.

[Isk80a] V. A. Iskovskikh. Birational automorphisms of three-dimensional algebraic varieties. J. Sov. Math., 13:815-868, 1980.

[Isk80b] V. A. Iskovskikh. Minimal models of rational surfaces over arbitrary fields. Math. USSR-Izv., 14(1):17-39, 1980.

[Isk87] V. A. Iskovskikh. On the rationality problem for conic bundles. Duke Math. J., 54:271-294, 1987.

[Isk91] V. A. Iskovskikh. On the rationality problem for conic bundles. Mat. Sb., 182(1):114-121, 1991.

[Isk95] V. A. Iskovskikh. On the rationality problem for three-dimensional algebraic varieties fibered over del Pezzo surfaces. Proc. Steklov Inst. Math., 208:128-138, 1995. 
[Isk96a] V. A. Iskovskikh. Factorization of birational mappings of rational surfaces from the point of view of Mori theory. Russian Math. Surveys, 51(4):585-652, 1996.

[Isk96b] V. A. Iskovskikh. On a rationality criterion for conic bundles. Mat. Sb., 187(7):75-92, 1996.

[Isk01] V. A. Iskovskikh. Birational rigidity of Fano hypersurfaces in the framework of Mori theory. Russian Math. Surveys, 56(2):207-291, 2001.

[Isk03] V. A. Iskovskikh. b-divisors and Shokurov functional algebras. Proc. Steklov Math. Inst., 240:4-15, 2003.

[Isk04] Vasilij A. Iskovskikh. On the Noether-Fano inequalities. In The Fano Conference, pages 25-35. Univ. Torino, Turin, 2004.

[Jac96] Nathan Jacobson. Finite-dimensional division algebras over fields. Berlin: Springer, 1996.

[JPR11] Priska Jahnke, Thomas Peternell, and Ivo Radloff. Threefolds with big and nef anticanonical bundles II. Cent. Eur. J. Math., 9(3):449488, 2011.

[Kac97] Yasuyuki Kachi. Extremal contractions from 4-dimensional manifolds to 3-folds. Ann. Scuola Norm. Sup. Pisa Cl. Sci. (4), 24(1):63$131,1997$.

[Kan01] S. Kantor. Die Typen der linearen Komplexe rationaler Kurven im $R_{r}$. Am. J. Math., 23:1-28, 1901.

[Kan01] Ming-chang Kang. The rationality problem of finite group actions. In First International Congress of Chinese Mathematicians (Beijing, 1998), volume 20 of AMS/IP Stud. Adv. Math., pages 211-218. Amer. Math. Soc., Providence, RI, 2001.

[Kaw88] Yujiro Kawamata. Crepant blowing-up of 3-dimensional canonical singularities and its application to degenerations of surfaces. Ann. of Math. (2), 127(1):93-163, 1988.

[Kaw92] Yujiro Kawamata. Boundedness of Q-Fano threefolds. In Proceedings of the International Conference on Algebra, Part 3 (Novosibirsk, 1989), volume 131 of Contemp. Math., pages 439-445, Providence, RI, 1992. Amer. Math. Soc.

[Kaw93] Yujiro Kawamata. The minimal discrepancy coefficients of terminal singularities in dimension three (Appendix to V.V. Shokurov's paper "3-fold log flips"). Russ. Acad. Sci., Izv., Math., 40(1):193-195, 1993.

[Kaw96] Yujiro Kawamata. Divisorial contractions to 3-dimensional terminal quotient singularities. In Higher-dimensional complex varieties (Trento, 1994), pages 241-246. de Gruyter, Berlin, 1996.

[KM92] János Kollár and Shigefumi Mori. Classification of three-dimensional flips. J. Amer. Math. Soc., 5(3):533-703, 1992.

[KM98] János Kollár and Shigefumi Mori. Birational geometry of algebraic varieties, volume 134 of Cambridge Tracts in Mathematics. Cambridge University Press, Cambridge, 1998. With the collaboration of C. H. Clemens and A. Corti, Translated from the 1998 Japanese original.

[KM17] János Kollár and Massimiliano Mella. Quadratic families of elliptic curves and unirationality of degree 1 conic bundles. Am. J. Math., 139(4):915-936, 2017. 
[KMM87] Yujiro Kawamata, Katsumi Matsuda, and Kenji Matsuki. Introduction to the minimal model problem. In Algebraic geometry, Sendai, 1985, volume 10 of Adv. Stud. Pure Math., pages 283-360. NorthHolland, Amsterdam, 1987.

[KMM92] János Kollár, Yoichi Miyaoka, and Shigefumi Mori. Rationally connected varieties. J. Algebraic Geom., 1(3):429-448, 1992.

[Kol86] János Kollár. Higher direct images of dualizing sheaves. I. Ann. of Math. (2), 123(1):11-42, 1986.

[Kol89] János Kollár. Flops. Nagoya Math. J., 113:15-36, 1989.

[Kol95] János Kollár. Nonrational hypersurfaces. J. Amer. Math. Soc., 8(1):241-249, 1995.

[Kol97] János Kollár. Polynomials with integral coefficients, equivalent to a given polynomial. Electron. Res. Announc. Amer. Math. Soc., 3:17$27,1997$.

[Kol02] János Kollár. Unirationality of cubic hypersurfaces. J. Inst. Math. Jussieu, 1(3):467-476, 2002.

[Kol17] János Kollár. Conic bundles that are not birational to numerical Calabi-Yau pairs. Épijournal de Géométrie Algébrique, 1, September 2017.

[KPS18] Alexander Kuznetsov, Yuri Prokhorov, and Constantin Shramov. Hilbert schemes of lines and conics and automorphism groups of Fano threefolds. Japanese J. Math., 13(1):109-185, 2018.

[Kry16] Igor Krylov. Birational geometry of del Pezzo fibrations with terminal quotient singularities. Arxiv e-print, 1606.03252, 2016.

[Kry18] Igor Krylov. Rationally connected non-Fano type varieties. European J. Math., 2018.

[KSC04] János Kollár, Karen E. Smith, and Alessio Corti. Rational and nearly rational varieties, volume 92 of Cambridge Studies in Advanced Mathematics. Cambridge University Press, Cambridge, 2004.

[KST89] B. Ė. Kunyavskiĭ, A. N. Skorobogatov, and M. A. Tsfasman. Del Pezzo surfaces of degree four. Mém. Soc. Math. France (N.S.), $37: 113,1989$.

[Kuz15] A. G. Kuznetsov. On Küchle varieties with Picard number greater than 1. Izv. Math., 79(4):698-709, 2015.

[Log17] Konstantin Loginov. Standard models of degree 1 del Pezzo fibrations. Arxiv e-print, 1710.02482, 2017.

[Man67] Yu. I. Manin. Rational surfaces over perfect fields. II. Mat. Sb. (N.S.), 72 (114):161-192, 1967.

[Man74] Yu. I. Manin. Cubic forms: algebra, geometry, arithmetic. NorthHolland Publishing Co., Amsterdam, 1974. Translated from the Russian by M. Hazewinkel, North-Holland Mathematical Library, Vol. 4 .

[Mas76] Leon Masiewicki. Universal properties on Prym varieties with an application to algebraic curves of genus five. Trans. Am. Math. Soc., 222:221-240, 1976.

[Mat02] Kenji Matsuki. Introduction to the Mori program. Universitext. Springer-Verlag, New York, 2002.

[Mel14] Massimiliano Mella. On the unirationality of 3-fold conic bundles. Arxiv e-print, 1403.7055, 2014. 
[Mer81] A.S. Merkur'ev. On the norm residue symbol of degree 2. Sov. Math., Dokl., 24:546-551, 1981.

[Mil60] Tomaso Millevoi. Sulla classificazione cremoniana delle congruenze di curve razionali dello spazio. Rend. Semin. Mat. Univ. Padova, 30:194-214, 1960.

[Miy83] Masayoshi Miyanishi. Algebraic methods in the theory of algebraic threefolds - surrounding the works of Iskovskikh, Mori and Sarkisov. In Algebraic varieties and analytic varieties (Tokyo, 1981), volume 1 of Adv. Stud. Pure Math., pages 69-99, Amsterdam, 1983. NorthHolland.

[MM86] Yoichi Miyaoka and Shigefumi Mori. A numerical criterion for uniruledness. Ann. of Math. (2), 124(1):65-69, 1986.

[MM82] Shigefumi Mori and Shigeru Mukai. Classification of Fano 3-folds with $B_{2} \geq 2$. Manuscripta Math., 36(2):147-162, 1981/82. Erratum: Manuscripta Math. 110 (2003), 407.

[Mor82] S. Mori. Threefolds whose canonical bundles are not numerically effective. Ann. Math., 115:133-176, 1982.

[Mor85] David R. Morrison. The birational geometry of surfaces with rational double points. Math. Ann., 271(3):415-438, 1985.

[Mor88] Shigefumi Mori. Flip theorem and the existence of minimal models for 3-folds. J. Amer. Math. Soc., 1(1):117-253, 1988.

[MP08a] Shigefumi Mori and Yuri Prokhorov. On Q-conic bundles. Publ. Res. Inst. Math. Sci., 44(2):315-369, 2008.

[MP08b] Shigefumi Mori and Yuri Prokhorov. On Q-conic bundles. II. Publ. Res. Inst. Math. Sci., 44(3):955-971, 2008.

[MP09] S. Mori and Yu. Prokhorov. On Q-conic bundles, III. Publ. Res. Inst. Math. Sci., 45(3):787-810, 2009.

[MP11] S. Mori and Yu. Prokhorov. Threefold extremal contractions of type IA. Kyoto J. Math., 51(2):393-438, 2011.

[MP14] S. Mori and Yu. Prokhorov. Threefold extremal contractions of types (IC) and (IIB). Proc. Edinburgh Math. Soc., 57(1):231-252, 2014.

[MP16] S. Mori and Yu. Prokhorov. Threefold extremal contractions of type (IIA), I. Izv. Math., 80(5):884-909, 2016.

[MP17] Shigefumi Mori and Yuri Prokhorov. Threefold extremal contractions of type (IIA), II. ArXiv e-print, 1707.05047, 2017. to appear in Festschrift in honour of Nigel Hitchin, (2018), Oxford University Press.

[Mum71] David Mumford. Theta characteristics of an algebraic curve. Ann. Sci. École Norm. Sup. (4), 4:181-192, 1971.

[Mum74] David Mumford. Prym varieties. I. In Contributions to analysis (a collection of papers dedicated to Lipman Bers), pages 325-350. Academic Press, New York, 1974.

[MZ88] M. Miyanishi and D.-Q. Zhang. Gorenstein log del Pezzo surfaces of rank one. J. Algebra, 118(1):63-84, 1988.

[Pan80] I.A. Panin. The rationality of bundles of conics with degeneration curves of degree five and even theta-characteristic. Zap. Nauchn. Semin. Leningr. Otd. Mat. Inst. Steklova, 103:100-106, 1980. (in Russian). 
[Par01] Jihun Park. Birational maps of del Pezzo fibrations. J. Reine Angew. Math., 538:213-221, 2001.

[Par03] Jihun Park. A note on del Pezzo fibrations of degree 1. Commun. Algebra, 31(12):5755-5768, 2003.

[PCS05] V.V. Przhiyalkovskij, I.A. Chel'tsov, and K.A. Shramov. Hyperelliptic and trigonal Fano threefolds. Izv. Math., 69(2):365-421, 2005.

[Pio07] Jens Piontkowski. Theta-characteristics on singular curves. J. Lond. Math. Soc., II. Ser., 75(2):479-494, 2007.

[Pop15] Vladimir L. Popov. Finite subgroups of diffeomorphism groups. Proc. Steklov Institute of Mathematics, 289(1):221-226, 2015.

[Pop17] V. L. Popov. Borel subgroups of Cremona groups. Math. Notes, 102(1):60-67, 2017.

[PR16] Yuri Prokhorov and Miles Reid. On Q-Fano threefolds of Fano index 2. In Minimal Models and Extremal Rays (Kyoto 2011), volume 70 of Adv. Stud. Pure Math., pages 397-420. Mathematical Society of Japan, Kinokuniya, Tokyo, 2016.

[Pro97a] Yu. Prokhorov. On extremal contractions from threefolds to surfaces: the case of one non-Gorenstein point. In Birational algebraic geometry (Baltimore, MD, 1996), volume 207 of Contemp. Math., pages 119-141. Amer. Math. Soc., Providence, RI, 1997.

[Pro97b] Yu. Prokhorov. On the complementability of the canonical divisor for Mori fibrations on conics. Sbornik. Math., 188(11):1665-1685, 1997.

[Pro99a] Yu. Prokhorov. Mori conic bundles with a reduced log-terminal boundary. J. Math. Sci. (New York), 94(1):1051-1059, 1999.

[Pro99b] Yu. Prokhorov. On extremal contractions from threefolds to surfaces: the case of one non-Gorenstein point and a nonsingular base surface. J. Math. Sci. (New York), 95(1):1986-1995, 1999.

[Pro05] Yu. Prokhorov. On the degree of Fano threefolds with canonical Gorenstein singularities. Russian Acad. Sci. Sb. Math., 196(1):81$122,2005$.

[Pro08] Yu. Prokhorov. Gap conjecture for 3-dimensional canonical thresholds. J. Math. Sci. Univ. Tokyo, 15(4):449-459, 2008.

[Pro10] Yuri Prokhorov. Fields of invariants of finite linear groups. In Cohomological and geometric approaches to rationality problems, volume 282 of Progr. Math., pages 245-273. Birkhäuser Boston Inc., Boston, MA, 2010.

[Pro15a] Yu. Prokhorov. On G-Fano threefolds. Izv. Math, 79(4):795-808, 2015.

[Pro15b] Yu. Prokhorov. Rational surfaces, volume 24 of Lekts. Kursy NOC. Steklov Math. Inst., RAS, Moscow, 2015. in Russian.

[Pro15c] Yuri Prokhorov. On stable conjugacy of finite subgroups of the plane Cremona group, II. Michigan Math. J., 64(2):293-318, 2015.

[Pro16] Yu. Prokhorov. Q-Fano threefolds of index 7. Proc. Steklov Inst. Math., 294:139-153, 2016.

[Pro17] Yuri. Prokhorov. On the number of singular points of terminal factorial Fano threefolds. Math. Notes, 101(5-6):1068-1073, 2017.

[PS09] Yu. Prokhorov and V. V. Shokurov. Towards the second main theorem on complements. J. Algebraic Geom., 18(1):151-199, 2009. 
[PS16a] Yu. Prokhorov and C. Shramov. Finite groups of birational selfmaps of threefolds. ArXiv e-print, 1611.00789, 2016. to appear in Math. Res. Lett.

[PS16b] Yu. Prokhorov and C. Shramov. p-subgroups in the space Cremona group. ArXiv e-print, 1610.02990, 2016. to appear in Math. Nachrichten.

[PS16c] Yuri Prokhorov and Constantin Shramov. Jordan property for Cremona groups. Amer. J. Math., 138(2):403-418, 2016.

[PS16d] Victor Przyjalkowski and Constantin Shramov. Double quadrics with large automorphism groups. Proc. Steklov Inst. Math., 294:154-175, 2016.

[PS17] Yu. Prokhorov and C. Shramov. Jordan constant for Cremona group of rank 3. Moscow Math. J., 17(3):457-509, 2017.

[Puk98] A.V. Pukhlikov. Birational automorphisms of algebraic threefolds with a pencil of Del Pezzo surfaces. Izv. Math., 62(1):115-155, 1998.

[Puk00a] A. V. Pukhlikov. Fiber-wise birational correspondences. Math. Notes, 68(1):103-112, 2000.

[Puk00b] Aleksandr V. Pukhlikov. Essentials of the method of maximal singularities. In Explicit birational geometry of 3-folds, pages 73-100. Cambridge: Cambridge University Press, 2000.

[Puk13] Aleksandr Pukhlikov. Birationally rigid varieties, volume 190 of Mathematical Surveys and Monographs. American Mathematical Society, Providence, RI, 2013.

[Puk17] A. V. Pukhlikov. Birational geometry of algebraic varieties fibred into Fano double spaces. Izvestiya: Math., 81(3):618-644, 2017.

[PV83] Luciana Picco Botta and Alessandro Verra. The non rationality of the generic Enriques threefold. Compos. Math., 48:167-184, 1983.

[Rec74] Sevin Recillas. Jacobians of curves with $g_{4}^{1}$ 's are the Prym's of trigonal curves. Bol. Soc. Mat. Mex., II. Ser., 19:9-13, 1974.

[Rei86] Miles Reid. Surfaces of small degree. Math. Ann., 275(1):71-80, 1986.

[Rei87] Miles Reid. Young person's guide to canonical singularities. In Algebraic geometry, Bowdoin, 1985 (Brunswick, Maine, 1985), volume 46 of Proc. Sympos. Pure Math., pages 345-414. Amer. Math. Soc., Providence, RI, 1987.

[Rei91] M. Reid. Birational geometry of 3-folds according to Sarkisov. preprint, 1991.

[Sal84] David J. Saltman. Noether's problem over an algebraically closed field. Invent. Math., 77(1):71-84, 1984.

[Sar81] V. G. Sarkisov. Birational isomorphisms of conic bundles. Math. USSR, Izv., 17:177-202, 1981.

[Sar82] V. G. Sarkisov. On the structure of conic bundles. Math. USSR, Izv., 120:355-390, 1982.

[Sar87] V. G. Sarkisov. Clasification of birational automorphisms of conic bundles, I. Preprint I. V. Kurchatov Institute, IAE-4446/15, 1987.

[Sar89] V. G. Sarkisov. Birational maps of standard Q-Fano fiberings. Preprint I. V. Kurchatov Institute, IAE-4901/1, 1989.

[SB04] Nicholas I. Shepherd-Barron. Stably rational irrational varieties. In The Fano conference. Papers of the conference organized to commemorate the 50th anniversary of the death of Gino Fano (1871-1952), 
Torino, Italy, September 29-October 5, 2002, pages 693-700. Torino: Università di Torino, Dipartimento di Matematica, 2004.

[SC11] V. V. Shokurov and Sung Rak Choi. Geography of log models: theory and applications. Cent. European J. Math., 9(3):489-534, 2011.

[Sha91] I.R. Shafarevich. Lüroth's problem. Proc. Steklov Inst. Math., 183:241-246, 1991.

[Sho84] V. V. Shokurov. Prym varieties: theory and applications. Math. USSR Izvestiya, 23(1):83-147, 1984.

[Sho86] V. V. Shokurov. A nonvanishing theorem. Math. USSR Izvestiya, 26(3):591-604, 1986.

[Sho93] V. V. Shokurov. 3-fold log flips. Russ. Acad. Sci., Izv., Math., 40(1):95-202, 1993.

[Sho03] Vyacheslav V. Shokurov. Prelimiting flips. Proc. Steklov Inst. Math., 240:75-213, 2003.

[Sho13] V. V. Shokurov. Log Adjunction: effectiveness and positivity. Arxiv e-print, 1308.5160, 2013.

[Sho17] V V Shokurov. A criterion for semiampleness. Izvestiya: Mathematics, 81 (4):827(4):827-887, 2017.

[Sho82] V. V. Shokurov. Distinguishing Prymians from Jacobians. Invent. Math., 65(2):209-219, 1981/82.

[Shr06a] K. A. Shramov. Ascending chain condition for the set of canonical thresholds of toric varieties. Sb. Math., 197(4):623-631, 2006.

[Shr06b] K. A. Shramov. On the rationality of non-singular threefolds with a pencil of Del Pezzo surfaces of degree 4. Mat. Sb., 197(1):133-144, 2006.

[Sob02] I. V. Sobolev. Birational automorphisms of a class of varieties fibered by cubic surfaces. Izv. Math., 66(1):201-222, 2002.

[Ste11] D. A. Stepanov. Smooth three-dimensional canonical thresholds. Math. Notes, 90(2):265-278, 2011.

[STVA14] Cecília Salgado, Damiano Testa, and Anthony Várilly-Alvarado. On the unirationality of del Pezzo surfaces of degree 2. J. Lond. Math. Soc. (2), 90(1):121-139, 2014.

[Tot16] Burt Totaro. Hypersurfaces that are not stably rational. J. Am. Math. Soc., 29(3):883-891, 2016.

[Tre85] S.L. Tregub. Construction of a birational isomorphism of a cubic threefold and Fano variety of the first kind with $g=8$, associated with a normal rational curve of degree 4. Mosc. Univ. Math. Bull., 40(6):78-80, 1985.

[Tre90] S.L. Tregub. On conic bundles birationally equivalent to a threedimensional cubic. Russ. Math. Surv., 45(5):237-238, 1990.

[Tre14] Andrey S. Trepalin. Rationality of the quotient of $\mathbf{P}^{2}$ by finite group of automorphisms over arbitrary field of characteristic zero. Cent. Eur. J. Math., 12(2):229-239, 2014.

[Tre16] Andrey Trepalin. Quotients of conic bundles. Transform. Groups, 21(1):275-295, 2016.

[Tyu71] A. N. Tyurin. The geometry of the Fano surface of a nonsingular cubic $F \subset P^{4}$, and Torelli's theorems for Fano surfaces and cubics. Math. USSR-Izv., 5(3):517-546, 1971. 
[Tyu73] A. N. Tyurin. Five lectures on three-dimensional varieties. Russ. Math. Surv., 27(5):1-53, 1973.

[Tyu75] A. N. Tyurin. The intersection of quadrics. Russian Math. Surveys, 30(6):51-105, 1975.

[Tyu76] A. N. Tyurin. On an invariant of a net of quadrics. Math. USSR, Izv., 9:21-25, 1976.

[Tyu80] A. N. Tyurin. The middle Jacobian of three-dimensional varieties. $J$. Sov. Math., 13:707-745, 1980.

[Var86] Robert Varley. Weddle's surfaces, Humbert's curves, and a certain 4-dimensional abelian variety. Amer. J. Math., 108(4):931-952, 1986.

[Voi88] Claire Voisin. Sur la jacobienne intermédiaire du double solide d'indice deux. Duke Math. J., 57(2):629-646, 1988.

[Voi15] Claire Voisin. Unirational threefolds with no universal codimension 2 cycle. Invent. Math., 201(1):207-237, 2015.

[VY86] I. I. Voronovich and V. I. Yanchevskiı̌. Rational splitting fields of simple algebras and unirationality of conic bundles. Dokl. Akad. Nauk BSSR, 30(4):293-296, 380, 1986.

[Yan85] V. I. Yanchevski1. $K$-unirationality of conic bundles and splitting fields of simple central algebras. Dokl. Akad. Nauk BSSR, 29(12):1061-1064, 1148, 1985.

[Yan92] Vyacheslav I. Yanchevskiı̌. $K$-unirationality of conic bundles over large arithmetic fields. Astérisque, (209):16, 311-320, 1992. Journées Arithmétiques, 1991 (Geneva).

[Yas16] Egor Yasinsky. Subgroups of odd order in the real plane Cremona group. J. Algebra, 461(1):87-120, 2016.

[Yas17] Egor Yasinsky. The Jordan constant for Cremona group of rank 2. Bull. Korean Math. Soc., 54(5):1859-1871, 2017.

[Zag77] A. A. Zagorskiir. Three-dimensional conic bundles. Math. Notes, 21(6):420-427, 1977.

Steklov Mathematical Institute of Russian Academy of Sciences, Moscow, Russia

National Research University Higher School of Economics, Moscow, Russia

E-mail address: prokhoro@mi.ras.ru 